Revue des patrimoines

$21 \mid 2013$

De l'art de bâtir aux champs à la ferme moderne

\title{
La reconstruction des fermes dans le département de l'Aisne après 1918
}

Jean-Charles Cappronnier et Franck Delorme

\section{(2) OpenEdition}

Journals

Édition électronique

URL : http://journals.openedition.org/insitu/10403

DOI : $10.4000 /$ insitu. 10403

ISSN : 1630-7305

Éditeur

Ministère de la Culture

Référence électronique

Jean-Charles Cappronnier et Franck Delorme, "La reconstruction des fermes dans le département de I'Aisne après 1918 », In Situ [En ligne], 21 | 2013, mis en ligne le 17 juillet 2013, consulté le 14 novembre 2019. URL : http://journals.openedition.org/insitu/10403 ; DOI : 10.4000/insitu.10403

Ce document a été généré automatiquement le 14 novembre 2019.

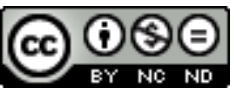

In Situ Revues des patrimoines est mis à disposition selon les termes de la licence Creative Commons Attribution - Pas d'Utilisation Commerciale - Pas de Modification 4.0 International. 


\section{La reconstruction des fermes dans le département de l'Aisne après 1918}

Jean-Charles Cappronnier et Franck Delorme

\section{Introduction}

Figure 1

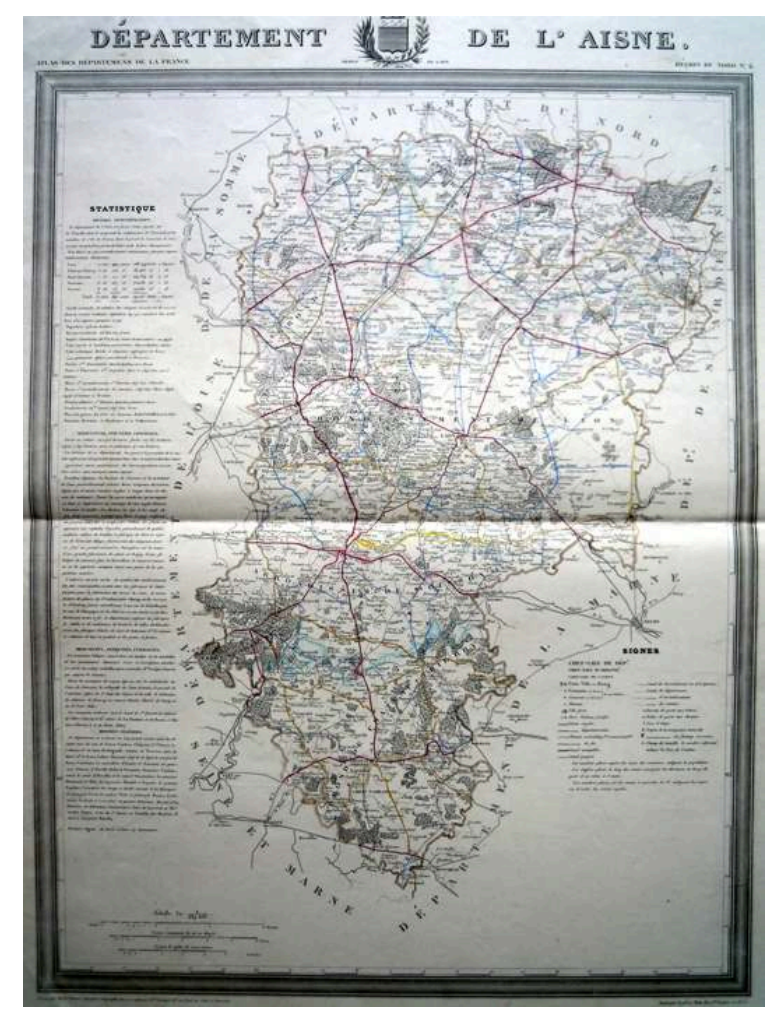

Carte du département de l'Aisne, par Alexis Dionnet, vers 1841. Archives nationales Pierrefitte, CP/F/ 14/10356/2/5

(c) Archives nationales. 
1 Le choix qui s'est porté sur le département de l'Aisne (fig. $\left.\mathbf{n}^{\circ} \mathbf{1}\right)$ repose sur les caractères spécifiques que revêt ce département dans le contexte de la reconstruction agricole d'après la Première Guerre mondiale. En premier lieu, ce département, dont la constitution administrative, en 1790, est parfaitement artificielle, révèle, de ce fait, une physionomie fortement contrastée. L'Aisne est, tout d'abord, et comme le révèle la carte géologique de la France, levée à la fin du XIX siècle et révisée entre 1897 et 1902, une véritable mosaïque géologique (fig. $\mathbf{n}^{\circ} \mathbf{2}$ ). Du bocage thiérachien à la Brie champenoise, des massifs forestiers du Valois aux plateaux du Soissonnais, le département, qui couvre une superficie de 7369 kilomètres carrés, illustre tous les substrats géologiques, offrant autant de matériaux potentiels disponibles : l'argile et la craie argileuse pour la Thiérache et le Vermandois, le calcaire lutétien pour le Soissonnais, le Valois et une partie du Laonnois, le grès ferrugineux, la pierre meulière, sans oublier le gypse - matière première des enduits au plâtre gros - pour le Tardenois et la vallée de la Marne, entre autres.

Figure 2

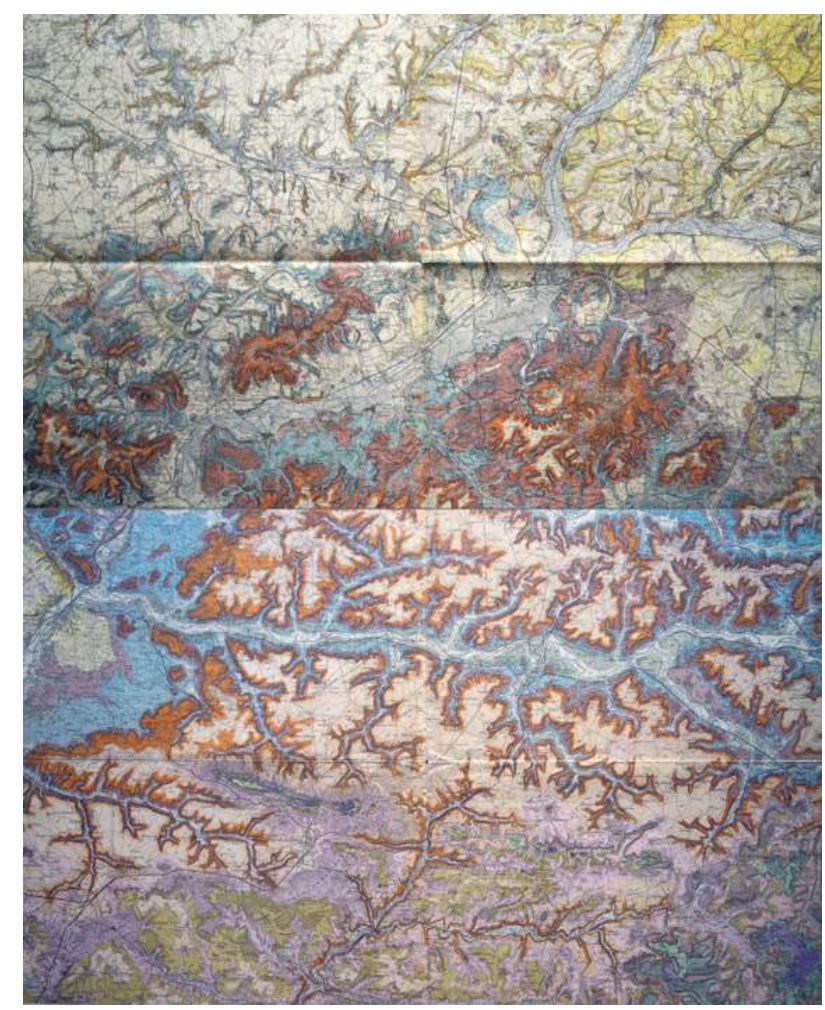

Carte géologique de l'Aisne, révisée entre 1897 et 1902 : détail du Laonnois et du Soissonnais Archives nationales Paris, CP/NN, supplément.

(c) Archives nationales.

2 Cette diversité géologique impose par conséquent au regard, à la veille de la Première Guerre mondiale, un paysage architectural tout aussi hétérogène. Le département axonien, pourvu depuis la Révolution d'un maillage serré de communes (840 en 1914), qui en fait encore aujourd'hui, malgré les regroupements et fusions consécutifs aux conflits, le deuxième département français en nombre de communes avec 816 entités, présente une forte emprise humaine sur le territoire. La variété architecturale correspond également aux multiples modes culturaux et à la physionomie 
historiquement fragmentée d'un département presque exclusivement rural, à l'exclusion du bassin industriel de Saint-Quentin et Chauny-Tergnier. Les anciennes fermes monastiques du Laonnois et du Soissonnais, dont l'organisation héritée du Moyen Âge, se prolonge avec de multiples réaménagements au cours du XIX ${ }^{\mathrm{e}}$ siècle, sont un des caractères saillants de ce paysage agricole à la veille de la Première Guerre mondiale ${ }^{1}$. Les expérimentations foncières et agronomiques du XIX siècle y sont également largement présentes, avec, probablement, parmi les plus célèbres, la ferme modèle de l'Arrouaise, construite à Oisy, en lisière du Vermandois et de la Thiérache, entre 1857 et 1860, à l'initiative de la famille Seillière ${ }^{2}$ (fig. $\mathbf{n}^{\circ} \mathbf{3}$ ).

Figure 3

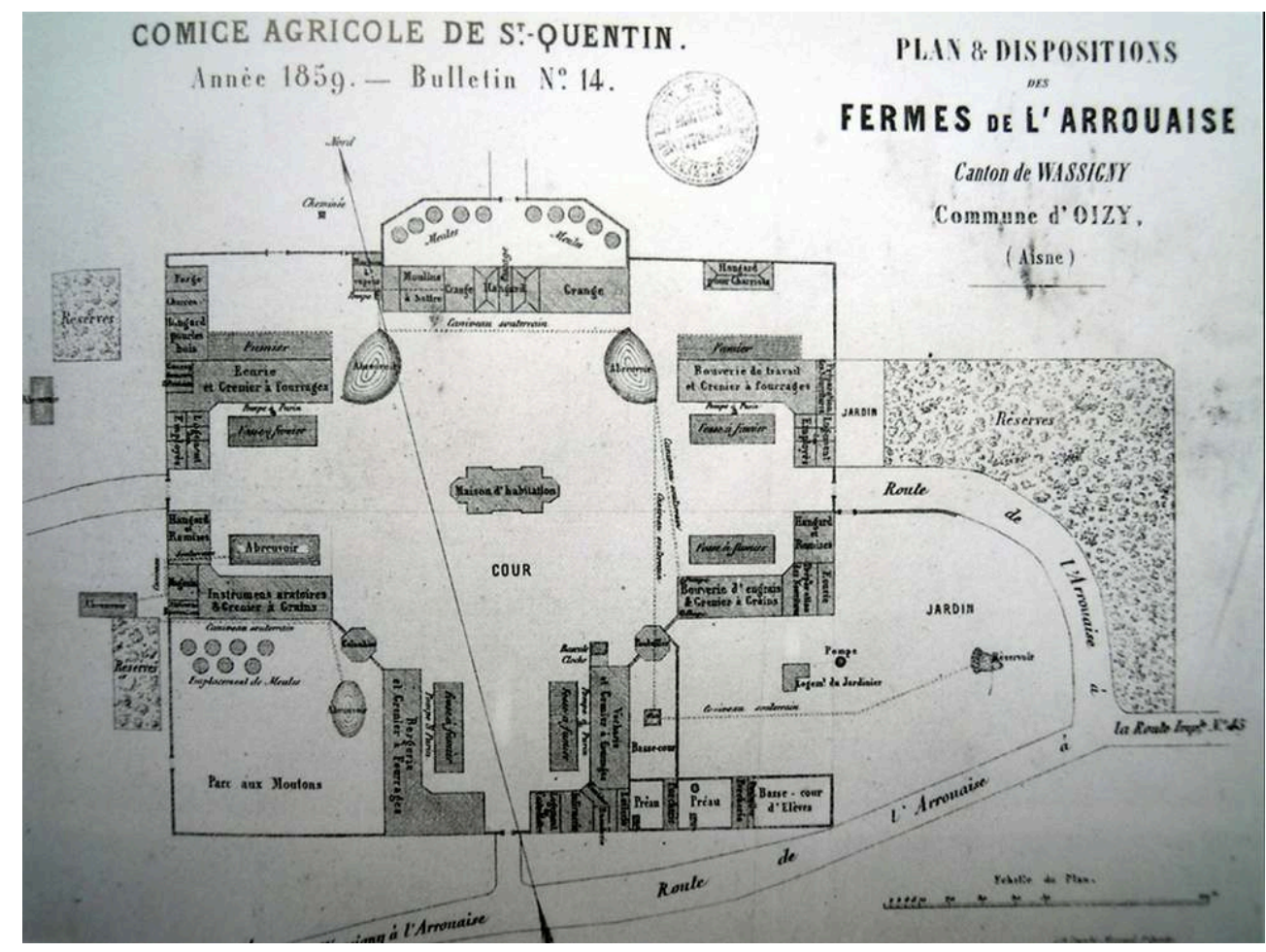

«Plan et disposition des fermes de l'Arrouaise », extrait du Bulletin du Comice agricole de Saint-Quentin, $n^{\circ} 14,1859$. Reproduit dans La Thiérache, Aisne : Sur une frontière de la France. Inventaire général des monuments et des richesses artistiques de la France / Association pour la généralisation de I'Inventaire régional en Picardie, 2001, p. 45. (Images du patrimoine, 207).

Repro. Cappronnier, Jean-Charles, Delorme, Franck. (c) Jean-Charles Cappronnier, Franck Delorme.

La sanglante balafre de la ligne de front (fig. $\mathbf{n}^{\circ} \mathbf{4}$ ), qui scinde le département pendant toute la durée de la guerre, les épisodes encore plus meurtriers de l'automne 1914 (fig. $n^{\circ} 5$ ) et de l'été 1918, en font un des départements les plus touchés par les destructions qui couvrent alors toute son étendue : 139 villages sont anéantis ; 461 détruits à plus de $50 \%$ et 235 endommagés. Seules six communes sortent indemnes du conflit. L'Aisne, qui, pas un instant au cours du conflit n'a été épargné par les destructions, devient un vaste "pays aplati », selon la formule de Dorgelès ${ }^{3}$, lui-même familier de ce territoire pendant et à l'issue de la guerre. Avec 113600 immeubles totalement sinistrés, l'Aisne se place au troisième rang des départements dévastés, après le Nord et la Somme. 
Figure 4

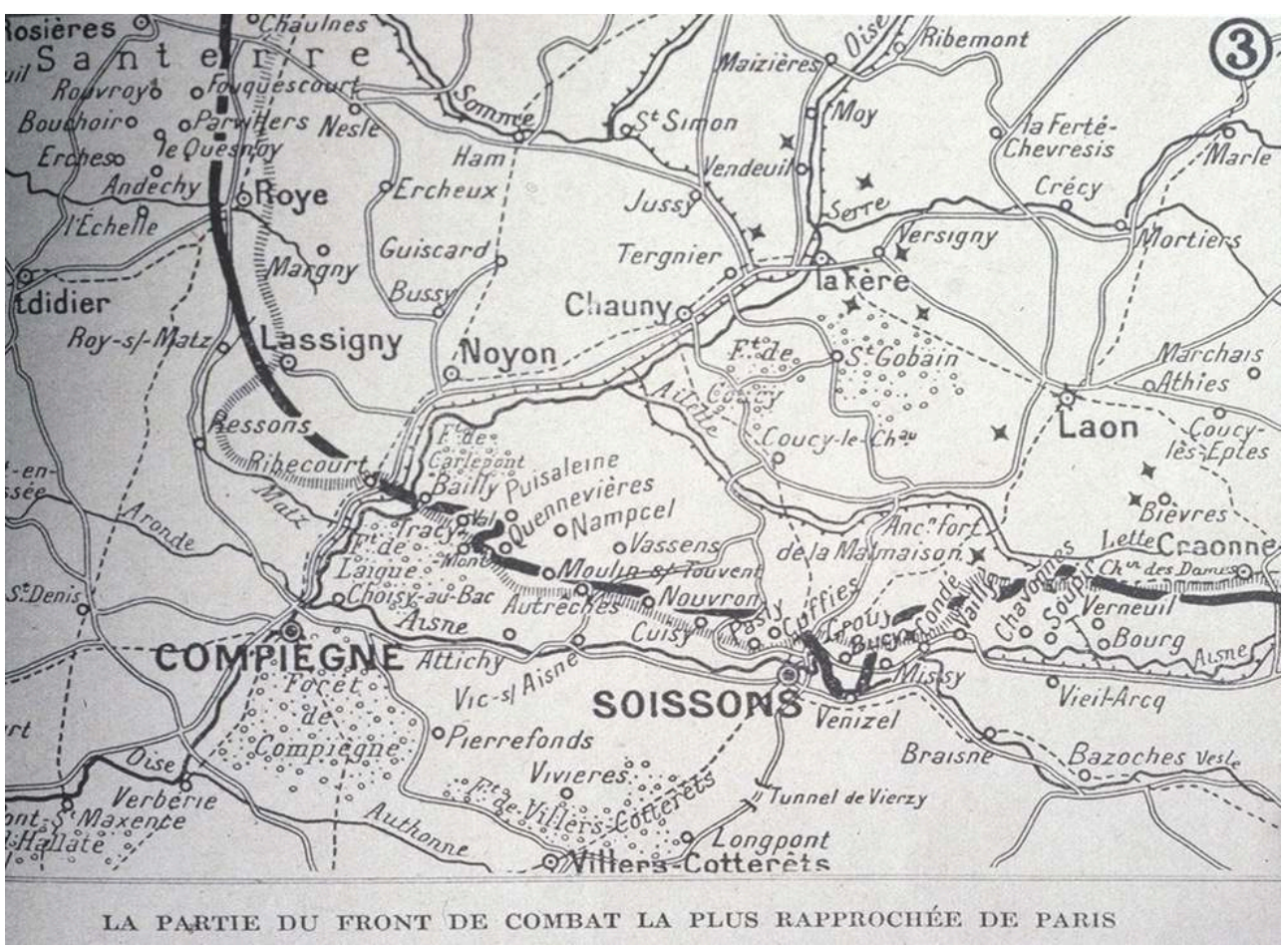

Carte de la ligne de front du Soissonnais en 1914-1915. Guide Michelin des champs de bataille. Paris Michelin Cartes et Guides.

Repro. Cappronnier, Jean-Charles, Delorme, Franck. (c) Jean-Charles Cappronnier, Franck Delorme.

Figure 5

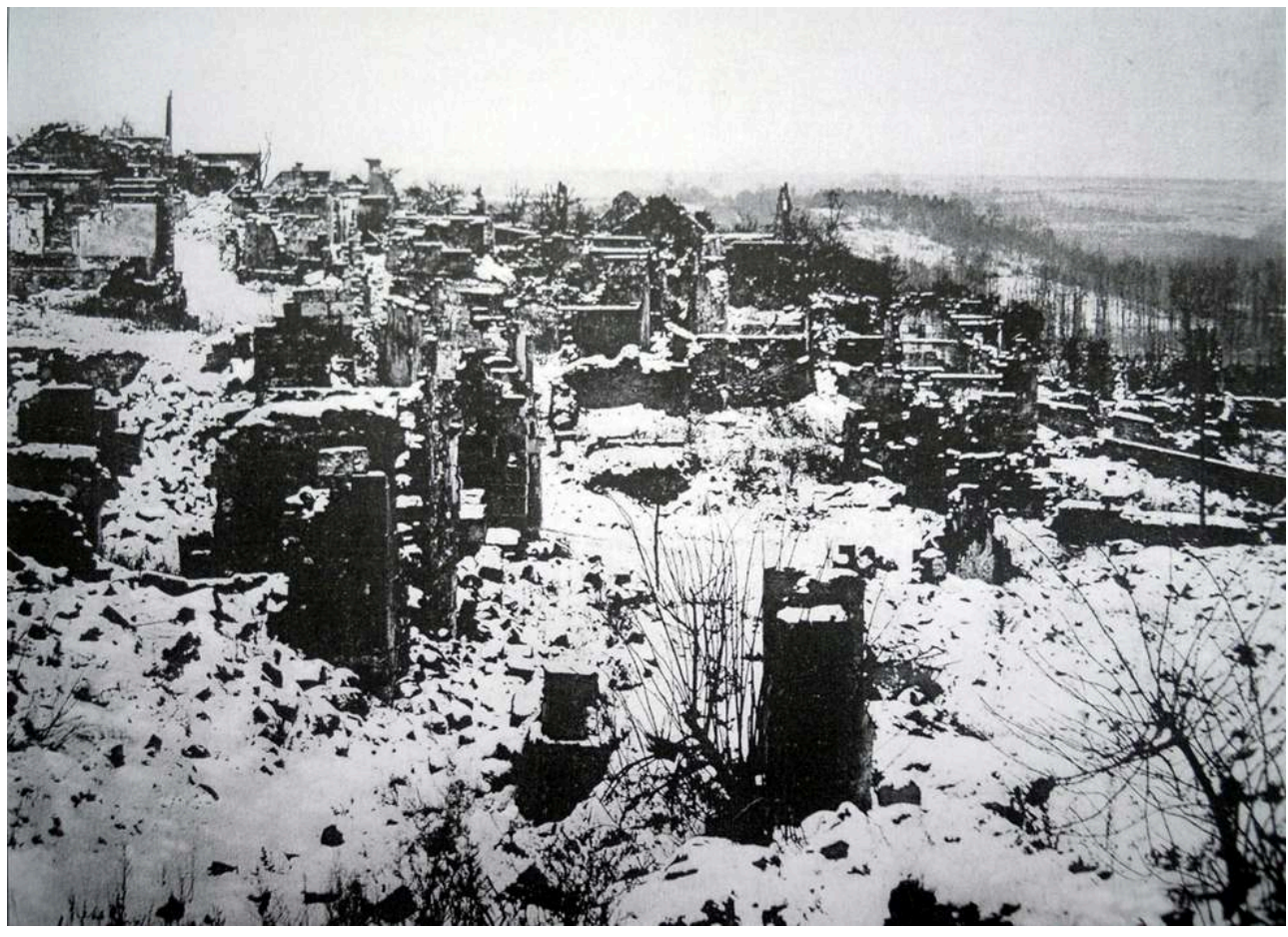

Les destructions. Le village de Nouvron-Vingré détruit en 1917. Photo de Jean-Baptiste Tournassoud. Collection particulière. 


\section{La question agricole à l'aune de la reconstruction dans l'Aisne}

4 Le retour au foyer détruit du paysan soldat, thème abondamment illustré pendant la guerre, avec, en particulier, les photographies entièrement fabriquées et composées du Service photographique de l'armée, comme certains autochromes de l'équipe Tournassoud ${ }^{4}$, installe dans la population, et dans la réalité administrative, l'idée que la reconstitution agricole est prioritaire. Dès l'automne 1914 et pendant toute l'année 1915, alors qu'un débat s'ouvre sur la restauration de la cathédrale de Reims ruinée par les obus, des écrivains comme le Belge Dumont-Wilden posent d'emblée les données du problème de la reconstruction rurale ${ }^{5}$.

Figure 6

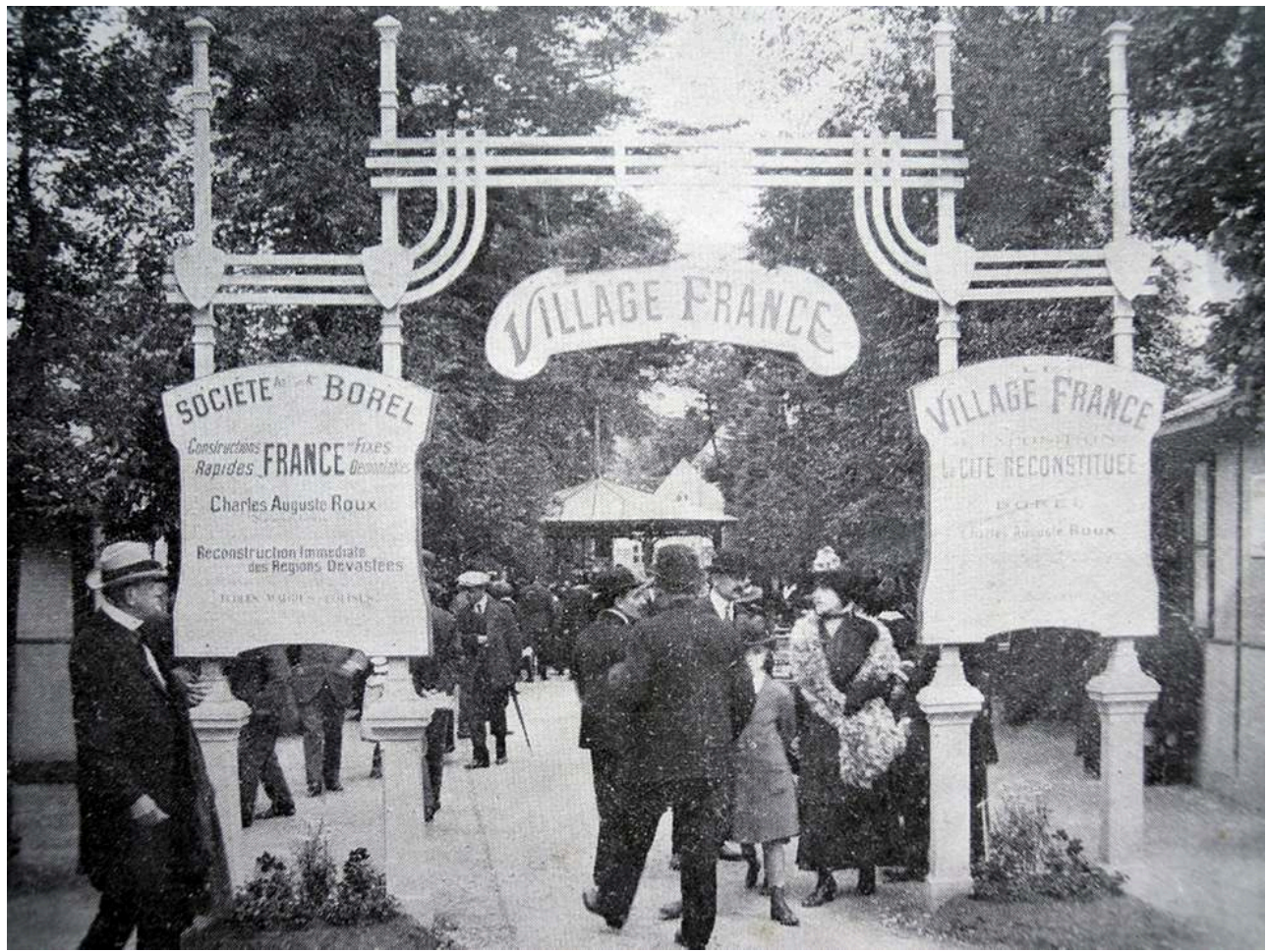

Les initiatives privées et associatives pendant la guerre. L'exposition de la Cité reconstituée. Porte d'entrée du village France, 1916. Photographie issue de "Une visite à la Cité reconstituée », Le Monde illustré, 24 juin 1916, p. 418

Repro. Cappronnier, Jean-Charles, Delorme, Franck. (c) Jean-Charles Cappronnier, Franck Delorme.

5 C'est dans un premier temps une large sphère privée, s'étendant des premières associations de sinistrés jusqu'aux sociétés professionnelles (hygiénistes et techniciens municipaux, urbanistes et architectes) qui s'empare de la question de la reconstruction et de ses multiples ramifications. En 1916, avec l'interminable prolongement de la guerre et de son cortège de destructions, les initiatives se multiplient. Aux mois de juin et juillet, une exposition intitulée «La Cité reconstituée » est organisée sur la terrasse des Tuileries à Paris. Ses chevilles ouvrières sont les industriels Édouard Tijou et Georges Risler (1853-1941), président du Musée social, auteur en 1923 d'un essai sur Le Travailleur agricole français. La manifestation promeut les travaux de l'ingénieur Charles 
Auguste Roux et la présentation de diverses entreprises, la construction provisoire et modulable appliquée à la reconstitution des régions dévastées et notamment destinée au monde agricole, avec la présentation du village «France » (fig. $\mathbf{n}^{\circ} \mathbf{6}$ ) et de la ferme «Agricola». L'exposition, également pilotée par l'Association des hygiénistes et techniciens municipaux, livre au public l'exemple du bourg de Vailly-sur-Aisne, comme étude de cas pour la reconstitution des régions dévastées. En 1917, l'affiche de L'Aisne dévastée, diffusée dans les préfectures, n'hésite pas à établir une symétrie entre deux traumatismes considérés comme équivalents : la ruine de la cathédrale de Soissons et celle d'un village axonien (fig. $\mathbf{n}^{\circ} \mathbf{7}$ ). Les projets et modèles types de constructions agricoles modulables et démontables connaissent un essor exceptionnel dans le sillage de cette manifestation, et, plus encore, en 1917, lorsque le retrait allemand sur la ligne Hindenburg laisse supposer une fin rapprochée du conflit et une réoccupation définitive d'un vaste territoire libéré des combats (fig. $\left.\mathbf{n}^{\circ} \mathbf{8}\right)$.

Figure 7

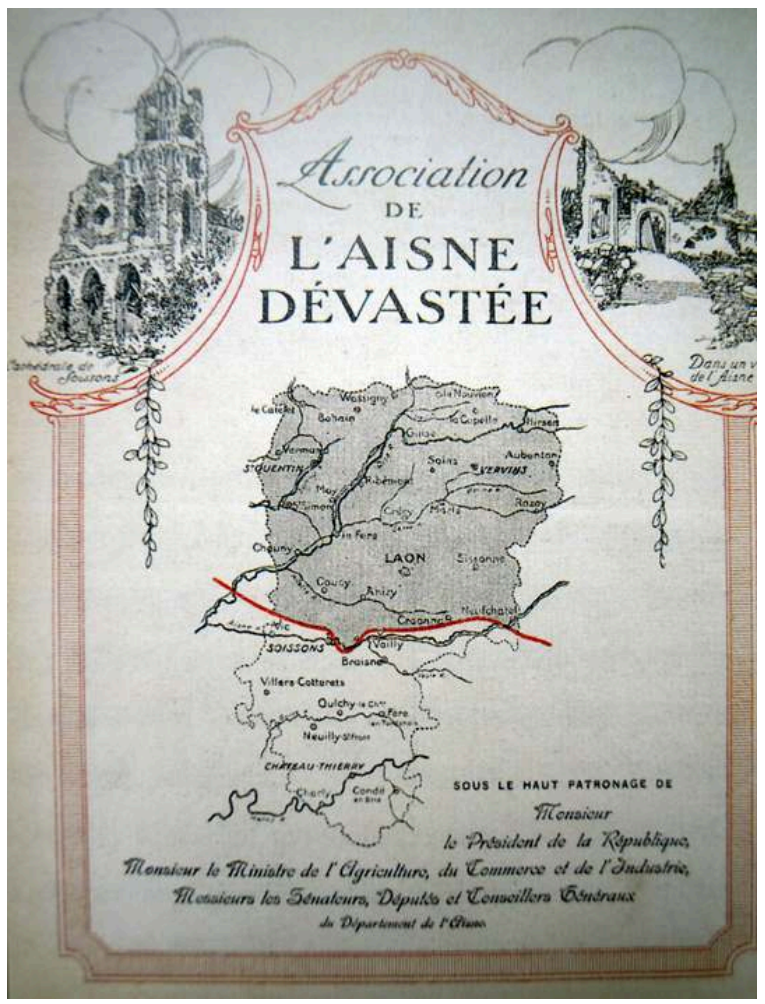

Les initiatives privées et associatives pendant la guerre. Affiche de "L'Aisne dévastée », circulaire diffusée auprès des préfectures par l'association L'Aisne dévastée, [1917]. Archives départementales de l'Aisne, $4^{\circ}$ br 462. Reproduite dans Reconstructions en Picardie après 1918. Paris : Réunion des Musées Nationaux, 2000, p. 293.

(C) Archives départementales de l'Aisne. 


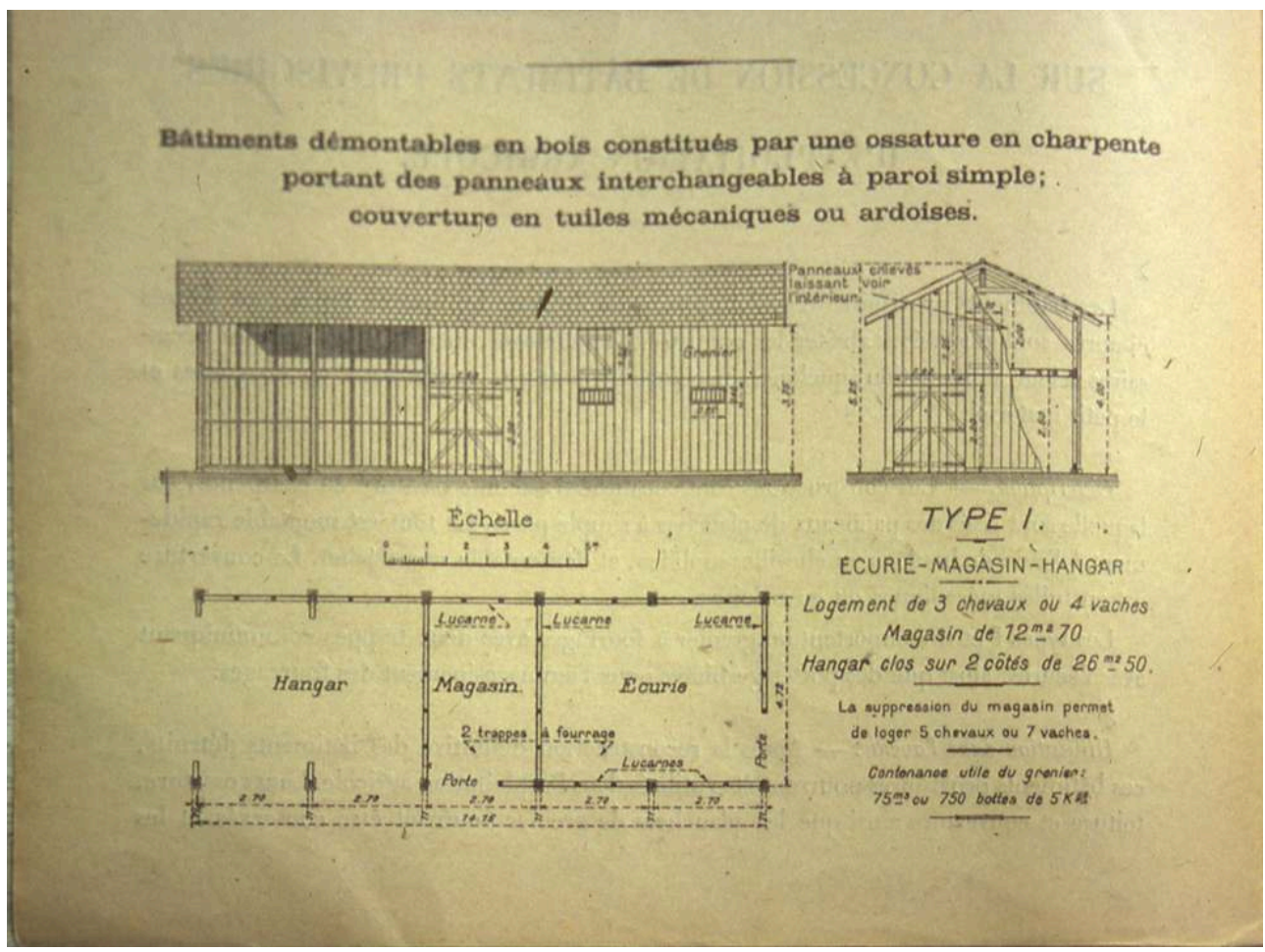

Modèles de constructions agricoles modulables et démontables. « Type I. Ecurie-magasin-hangar ».

(c) Archives départementales de la Somme.

Quant aux interventions de l'État, elles se manifestent de manière plus pragmatique et officielle à partir de 1917. Certes, dès le mois d'août 1915, l'architecte en chef des monuments historiques André Ventre (1874-1951) avait été chargé de plusieurs missions successives de relevés d'architecture rurale dans les départements soumis aux opérations militaires et aux destructions, mais hors des zones de combats. C'est ainsi que Ventre intervient dans l'Aisne avant les ultimes destructions de 1918, où il effectue, entre autres, des relevés d'une ferme de plateau à Chaudun (fig. $\mathbf{n}^{\circ} \mathbf{9}$ ), dans le SudSoissonnais, et d'une ferme et de maisons ouvrières à Épieds dans le Tardenois. Un certain nombre de ces dessins figuraient dans l'Exposition d'architecture régionale de 1917, prélude au célèbre concours de modèles de l'automne suivant où l'Aisne figure en bonne place au sein de la "Deuxième Région » : sur les 1499 projets rendus ${ }^{6}$, plusieurs projets axoniens sont primés, en particulier la ferme de moyenne culture de Pierre Guidetti (1878-1927) ou la maison de petit propriétaire rural dans l'Aisne de Pierre Sardou (1873-1952) (fig. $\left.\mathbf{n}^{\circ} 10\right)$. 
Figure 9

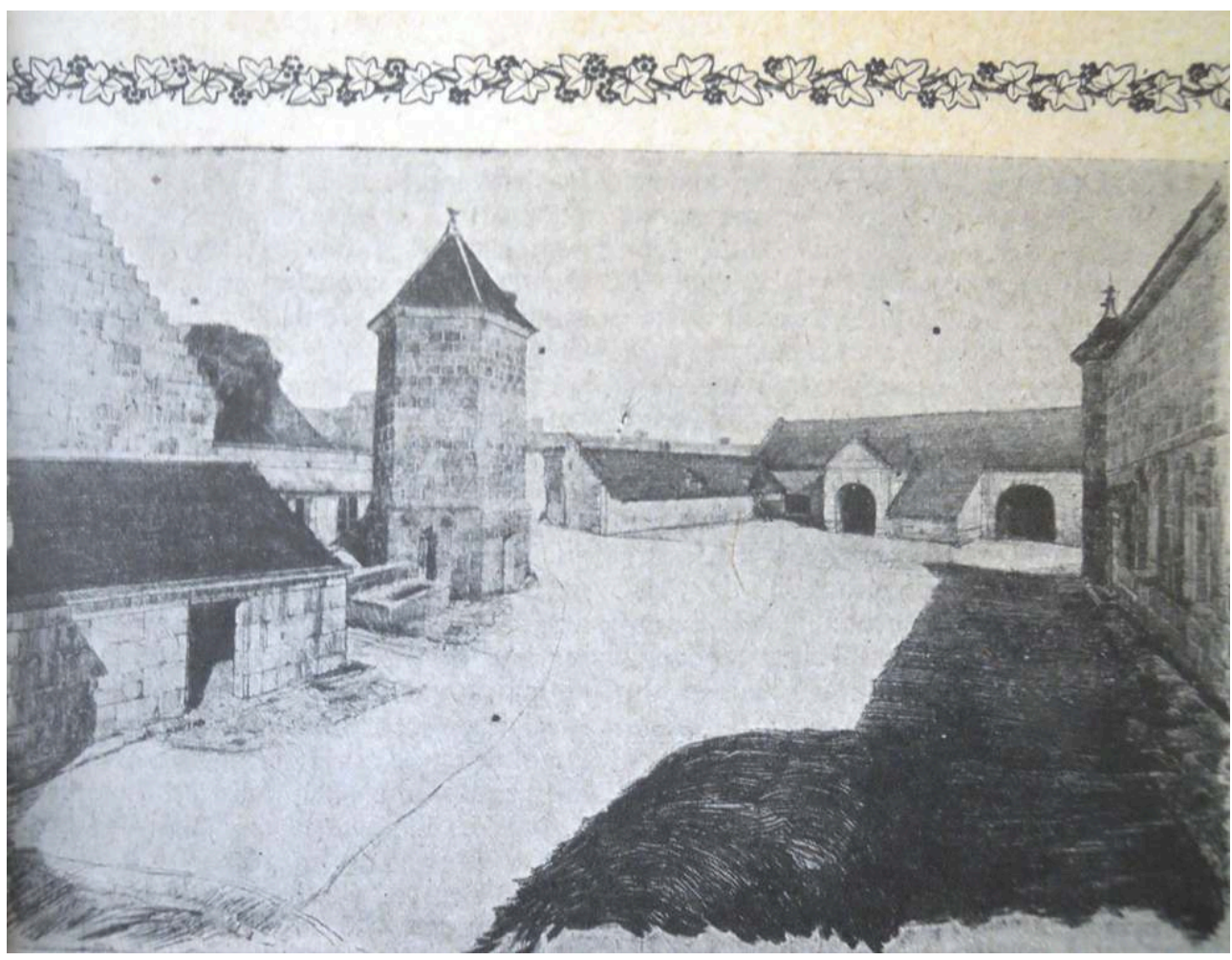

Les interventions de l'État. Les campagnes de relevés d'André Ventre. Ferme à Chaudun dessinée par Ventre, 1916 ou 1917. Plan reproduit dans Paul Léon, « La maison change avec la contrée », Lectures pour tous, $1^{\mathrm{er}}$ mars 1917.

Repro. Cappronnier, Jean-Charles, Delorme, Franck. ( ) Jean-Charles Cappronnier, Franck Delorme. 
Figure 10

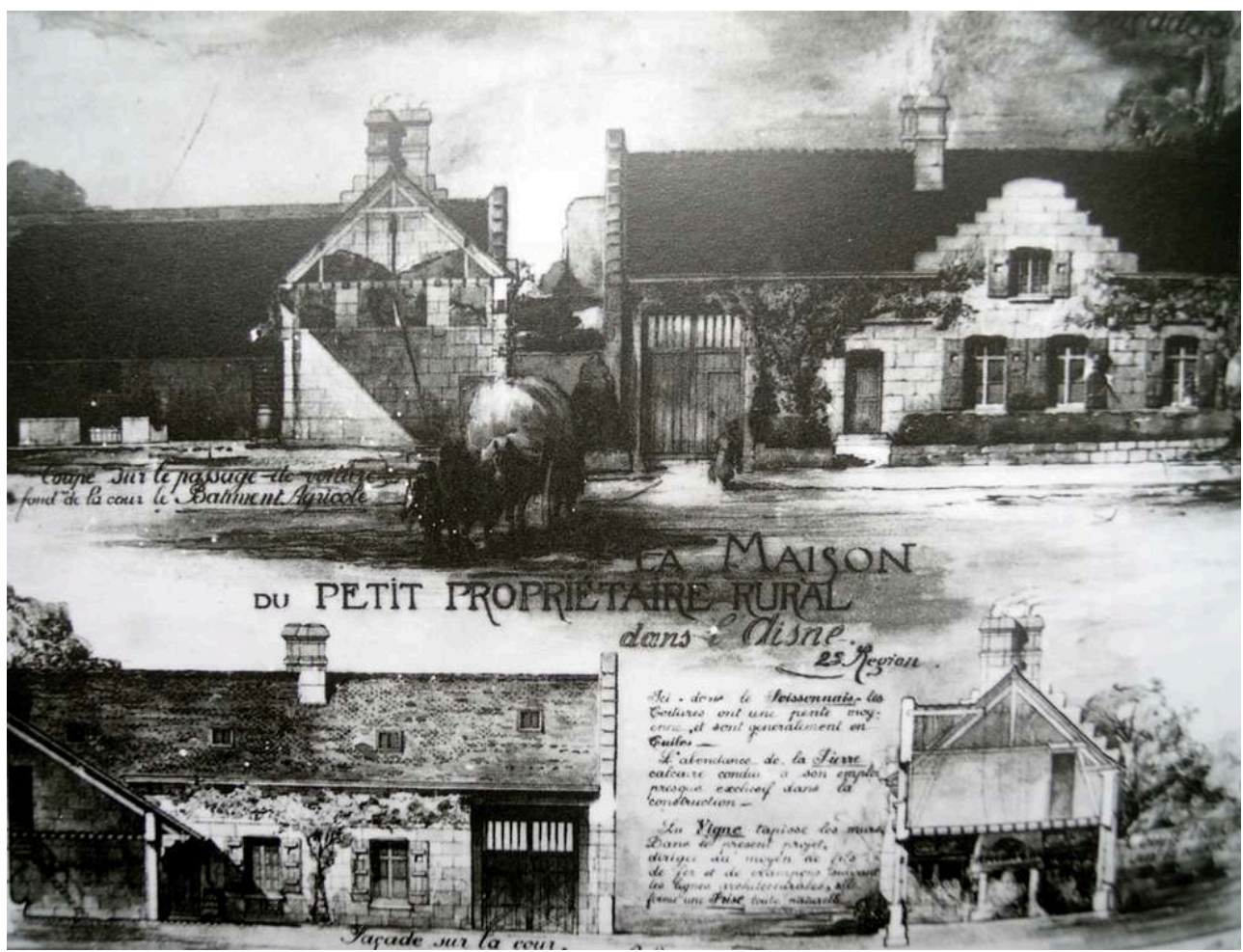

Le concours de 1917 : projet primé. Maison de petit propriétaire rural dans l'Aisne par Pierre Sardou. Reproduit dans Fermes et habitations rurales : projets primés au concours ouvert entre les architectes français. Paris : Massin, [1918].

Repro. Cappronnier, Jean-Charles, Delorme, Franck. (c) Jean-Charles Cappronnier, Franck Delorme.

7 Sont successivement créés, en 1916, le Comité interministériel pour la reconstitution des régions envahies - futur promoteur, avec les sociétés d'architectes, du concours de modèles - et, en août 1917, l'office de reconstitution agricole (ORA) des régions envahies, destiné à approvisionner les agriculteurs en outillage, équipements et matières premières, par l'intermédiaire d'entreprises tiers-mandataires. Ces deux créations engagent de manière institutionnelle et beaucoup plus résolue les services de l'État dans la voie d'une intervention plus directe dans l'organisation de la reconstitution. La même année, la création par Clémenceau d'un Commissariat général à l'agriculture, dont est titulaire le député socialiste Adéodat Compère-Morel (1872-1941) - qui, dans les années 1920 sera plusieurs fois rapporteur du budget de l'agriculture -, renforce cet interventionnisme, notamment en matière de gestion des terres abandonnées. Compère-Morel n'hésite pas à signer, en 1918, des articles dithyrambiques sur "La ferme de demain", où l'hygiène, la mécanisation et l'automatisation des tâches dessinent les contours de la future "usine agricole» (fig. $\left.\mathbf{n}^{\circ} 11\right)$. En 1920, il fonde l'hebdomadaire La Voix paysanne. 


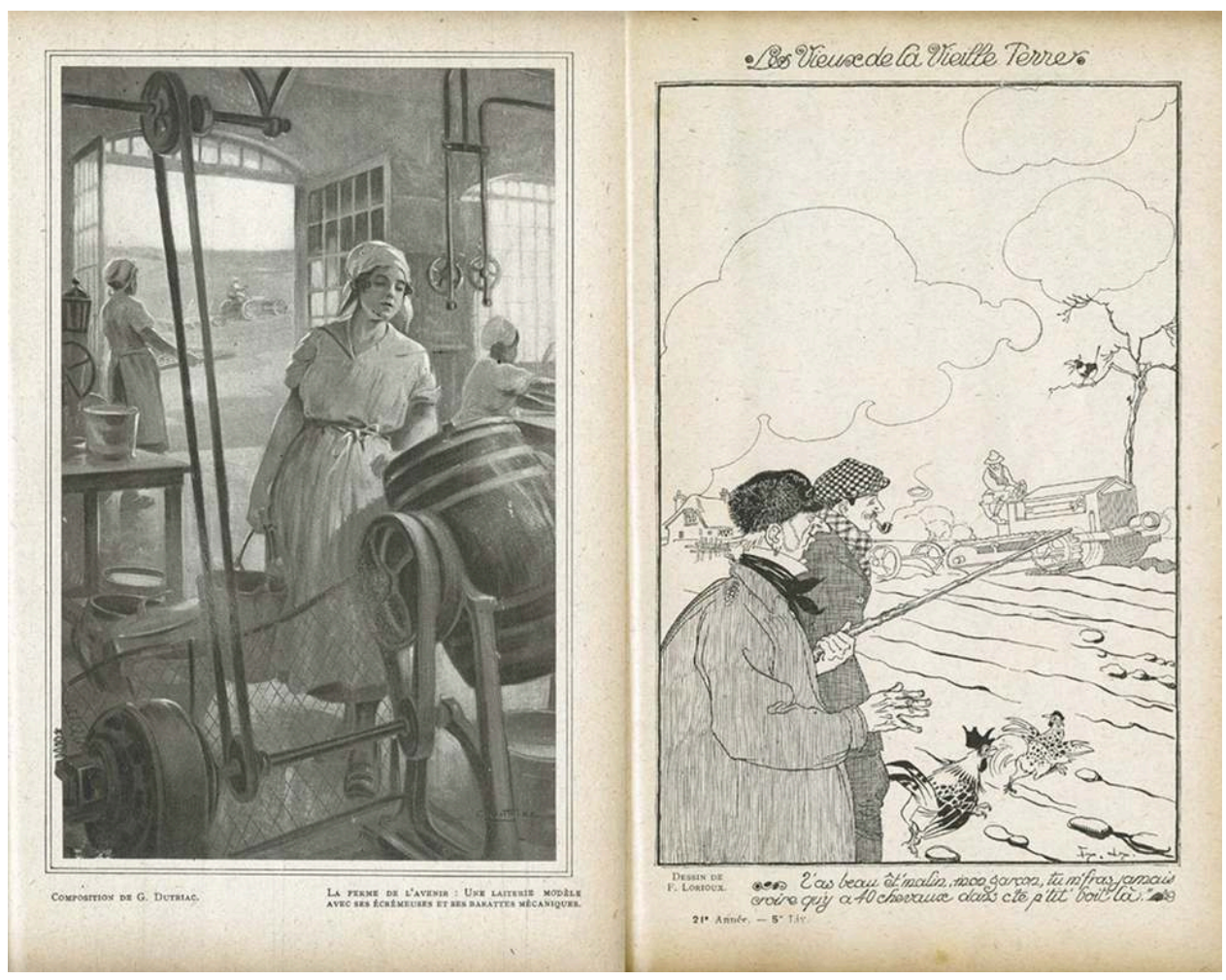

Le commissariat général à l'agriculture en 1918 : la « ferme de demain » de Compère-Morel. « Une laiterie moderne » et « Les vieux de la terre ». Dessins reproduits dans Lectures pour tous, $1^{\text {er }}$ décembre 1918.

Repro. Cappronnier, Jean-Charles, Delorme, Franck. @ Jean-Charles Cappronnier, Franck Delorme.

8 En cette fin de guerre, les publications abondent, accompagnant la mise en place d'un corpus réglementaire et législatif esquissé dès 1917. Trois textes de lois influent fortement sur l'organisation administrative de la reconstruction à venir. Tout d'abord, la loi Cornudet du 14 mars 1919, qui, au-delà de l'exigence des Plans d'aménagement, d'embellissement et d'extension bien connus, impose à toutes les communes sinistrées, et ce quelle que soit leur taille, de se doter d'un nouveau plan d'alignement mais aussi d'une "étude sommaire d'aménagement», premier véritable plan d'urbanisme à l'échelle de ces communes rurales sinistrées. L'établissement de ces plans est souvent confié aux architectes désignés par les coopératives civiles de reconstruction, comme par exemple Camille Lefèvre, pour le village de Leuilly-sous-Coucy (fig. $\left.\mathbf{n}^{\mathbf{0}} \mathbf{1 2}\right)$. Ensuite, la loi du 17 avril 1919, dite "Charte des sinistrés» qui établit et détermine les procédures d'indemnisation au titre des dommages de guerre, est suivie d'une kyrielle de textes réglementaires destinés à préciser les termes de la loi, ou à servir d'appui aux expertises menées par les architectes des sinistrés. C'est ainsi que le ministère des Régions libérées publie tout à la fois des séries de prix et des modèles types de constructions agricoles (fig. $\mathbf{n}^{\circ}{ }^{13}$ ) destinés à harmoniser et simplifier la rédaction des dossiers et l'évaluation des dommages. Enfin, la loi du 15 août 1920, réglementant les coopératives de reconstruction, reconnaît officiellement l'existence et le rôle de ces associations de sinistrés qui assureront la maitrise d'ouvrage de la plus grande partie des travaux de reconstruction, notamment dans le domaine agricole. 


\section{Figure 12}

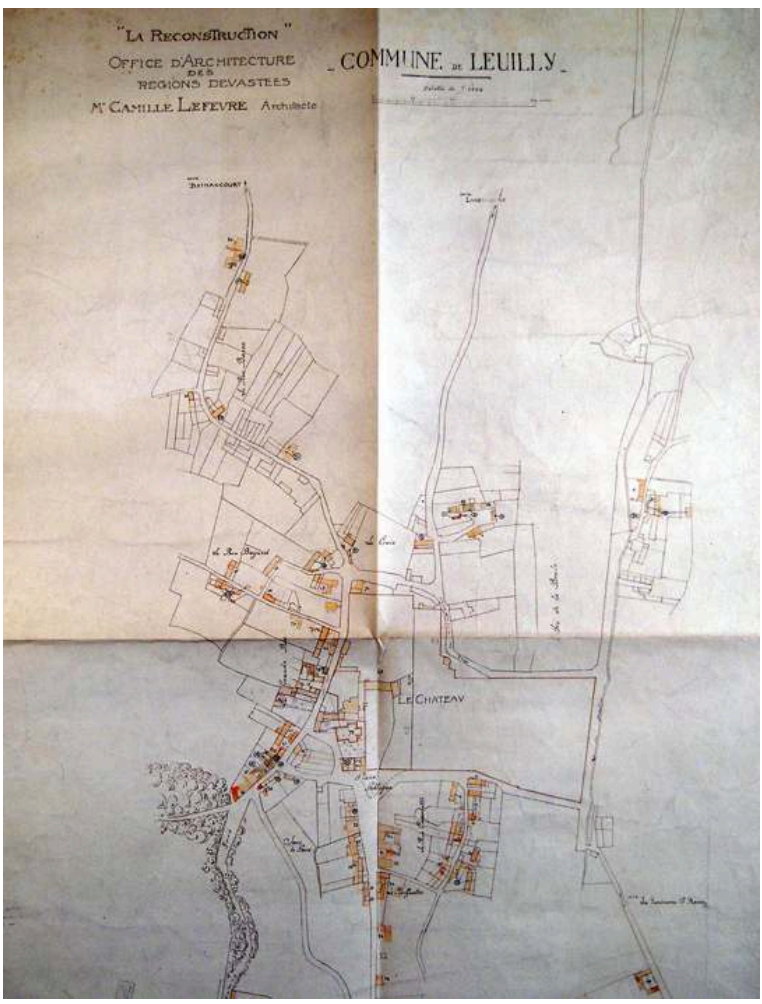

Le corpus réglementaire et ses ambigüités : la loi Cornudet et les esquisses sommaires d'aménagement. Plan de Leuilly-sous-Coucy par Camille Lefèvre, s.d., [1919]. Archives nationales de Pierrefitte, CP/VA/216, pièces 32 et 33.

(C) Archives nationales. 
Figure 13

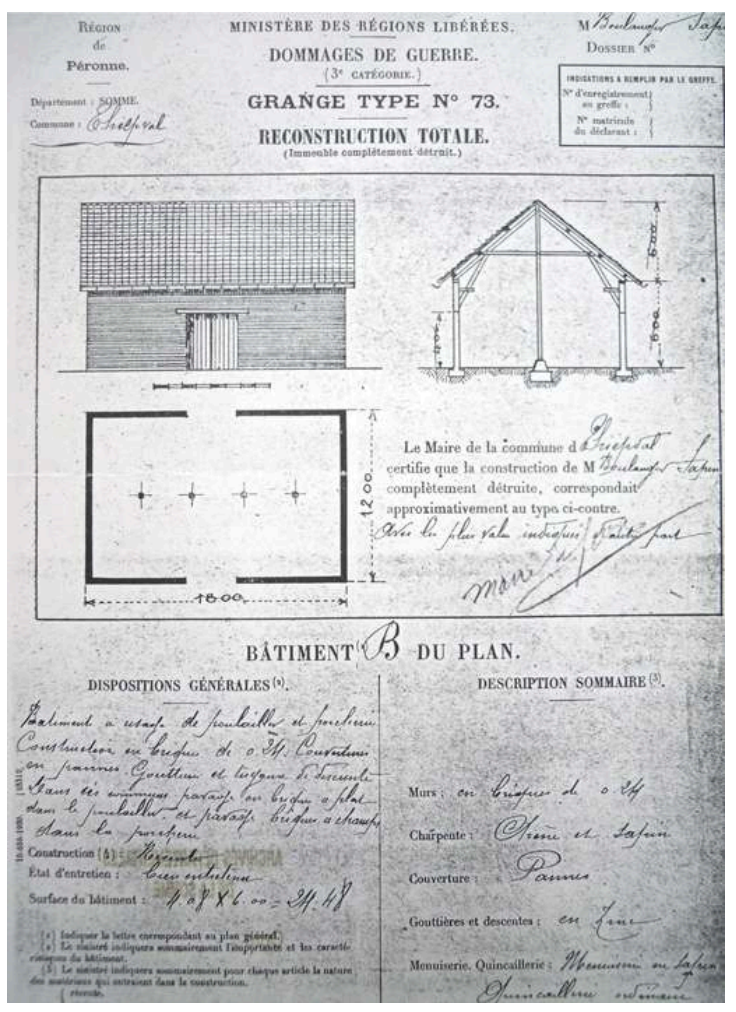

Le corpus réglementaire et ses ambigüités : la loi du 17 avril 1919. Plan-type de grange fourni par le Ministère des Régions libérées pour les expertises de Dommages de guerre. Archives départementales de la Somme, 10/R/1218.

(c) Archives départementales de la Somme.

\section{Les recherches en matière d'architecture agricole, avant et après la guerre de 1914-1918}




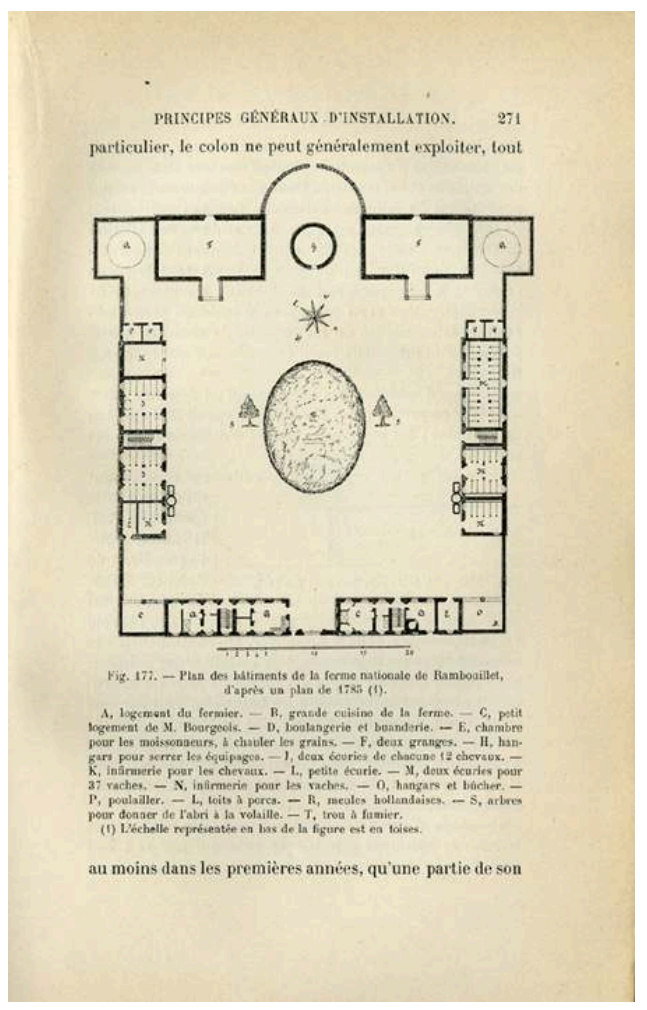

Plan de la bergerie impériale de Rambouillet (1785), extrait de Jacques Danguy, Constructions rurales, 1908.

Repro. Cappronnier, Jean-Charles, Delorme, Franck. @ Jean-Charles Cappronnier, Franck Delorme.

Dans le tome 4 de son ouvrage théorique Éléments et théorie de l'architecture publié en 1901, s'adressant aux architectes amenés à intervenir en milieu rural, voici la précision que Julien Guadet apporte: "À vrai dire, la construction rurale proprement dite n'existe que là où la propriété est assez vaste pour permettre une exploitation

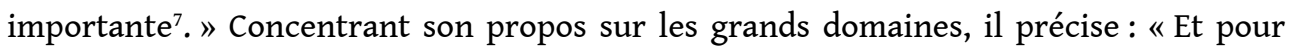
désigner l'ensemble des constructions élevées sur un domaine, je suivrai l'usage en me servant du mot La Ferme." Sans surprise, il fait remonter le développement des améliorations en matière d'architecture agricole, aux expériences menées depuis le milieu du XIX ${ }^{e}$ siècle, mettant surtout en avant l'attention portée à l'organisation du plan d'ensemble de la ferme : " Tout cela d'ailleurs a fait depuis un demi-siècle de très sensibles progrès; tandis que autrefois on édifiait un peu au hasard des bâtiments un peu à toute fin, on est arrivé à comprendre que des constructions bien aménagées pour leur objet spécial, hygiéniques et durables, constituent une économie judicieuse, et l'expérience a fixé les règles à observer dans chaque cas, pour chaque espèce d'animaux, presque pour chaque race dans chaque espèce. » À travers ces propos, on devine que Guadet renvoie aux grands exemples de fermes modèles comme par exemple la bergerie impériale de Rambouillet (1785) (fig. $\mathbf{n}^{\circ} \mathbf{1 4}$ ), les fermes modèles créées par l'ingénieur et industriel Armand Moisant dans ses domaines de Platé (fig. $\mathbf{n}^{\circ} 15$ ) ou de Thoriau, en Indre-et-Loire, à partir de $1878^{8}$, ou l'établissement de Grignon, ferme expérimentale de l'École nationale d'agriculture, édifiée à partir de $1870^{\circ}$. Nous pourrions également citer des exemples d'initiative privée comme la ferme modèle du château de Chaumont-sur-Loire conçue et réalisée par les architectes Paul Ernest

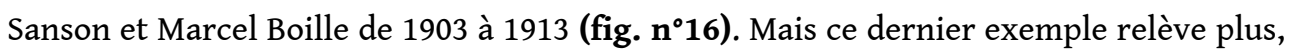


à notre avis, du cas d'un domaine aristocratique avec architecture de prestige que nous traiterons dans la troisième et dernière partie de l'étude.

Figure 15

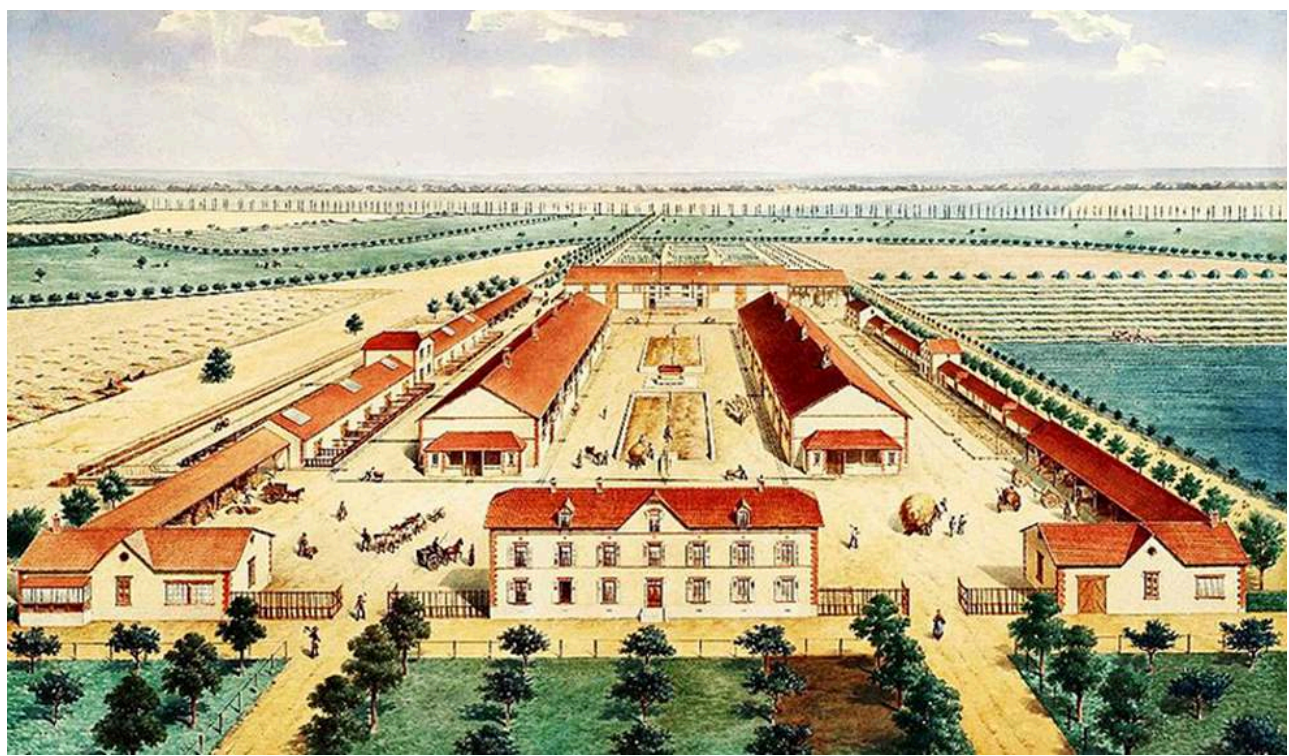

Perspective d'ensemble de la Ferme de Platé, d'Armand Moisant à Neuville-le-Roi dans I'Indre-et-Loire. Archives départementales d'Indre-et-Loire, fonds Moisant-Savey, 119/J.

(c) Archives départementales d'Indre-et-Loire.

\section{Figure 16}

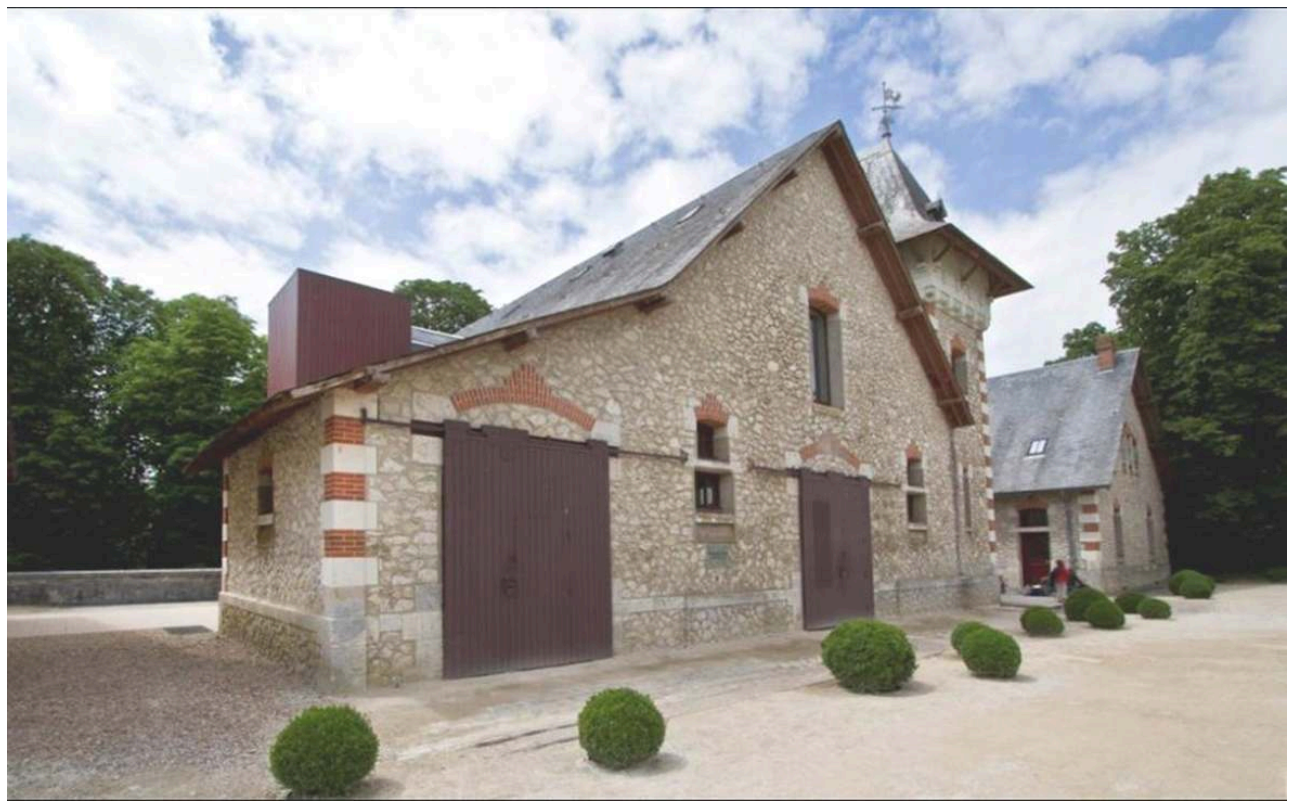

Vue du bâtiment du fenil de la Ferme du château de Chaumont-sur-Loire par Paul-Ernest Sanson et Marcel Boille.

Phot. Delorme, Franck. (c) Franck Delorme.

10 Les expériences sont donc nombreuses. Elles s'appuient sur les essais de théorisation à destination de différents publics, et les prolongent. Si le genre a connu ses premières heures de gloire dans les premières décennies du XIX ${ }^{e}$ siècle, pour une grande part dans 
le sillage des théories physiocratiques, il se renouvelle dans les dernières décennies du même siècle. La production littéraire est alors tout autant abondante, du Traité des constructions rurales et de leur disposition de Louis Bouchard-Huzard en 1858-1860 ${ }^{10}$ abondamment cité par Guadet -, aux Types de constructions rurales. Culture mixte, fermes, écuries, étables, bergeries, celliers, granges, citernes, réservoirs, etc. d'Émile Carlier en $1881^{11}$. D'autres entreprises, à visée technique et professionnelle, sont publiées à l'attention des grands cultivateurs comme des ouvrages de mise en pratique, tel le Traité élémentaire des constructions rurales rédigé en $1883^{12}$ par Jules Alexandre Grandvoinet, professeur de génie rural à l'Institut national agronomique, ou encore La Ferme moderne, traité des constructions rurales de $1903^{13}$ de Mars Abadie, ingénieur agronome, professeur de génie rural à l'École nationale d'agriculture de Rennes ${ }^{14}$. Pour notre étude, nous nous sommes appuyés sur le projet d'Encyclopédie agricole publiée en quarante volumes sous la direction du sous-directeur de l'Institut national agronomique G. Wéry, et plus particulièrement, au sein de la section $\mathrm{V}$ consacrée au génie agricole, sur le volume réservé aux Constructions rurales (fig. $\mathbf{n}^{\circ}{ }^{17}$ ). Publié en 1908, le texte est rédigé par l'ingénieur agronome Jacques Danguy, directeur des études à l'École nationale d'agriculture de Grignon. Le volume est structuré en deux parties, la première détaillant les techniques de construction en usage, la seconde traitant des différents bâtiments et locaux agricoles, de leur aménagement et de leur équipement. Enfin, à l'usage des architectes et de leur clientèle éclairée, et suivant le modèle d'Eugène Emmanuel Viollet-le-Duc et de son Histoire d'une maison, Félix Narjoux publie en 1882, Histoire d'une ferme ${ }^{15}$ (fig. $\mathbf{n}^{\circ} \mathbf{1 8}$ ).

Figure 17

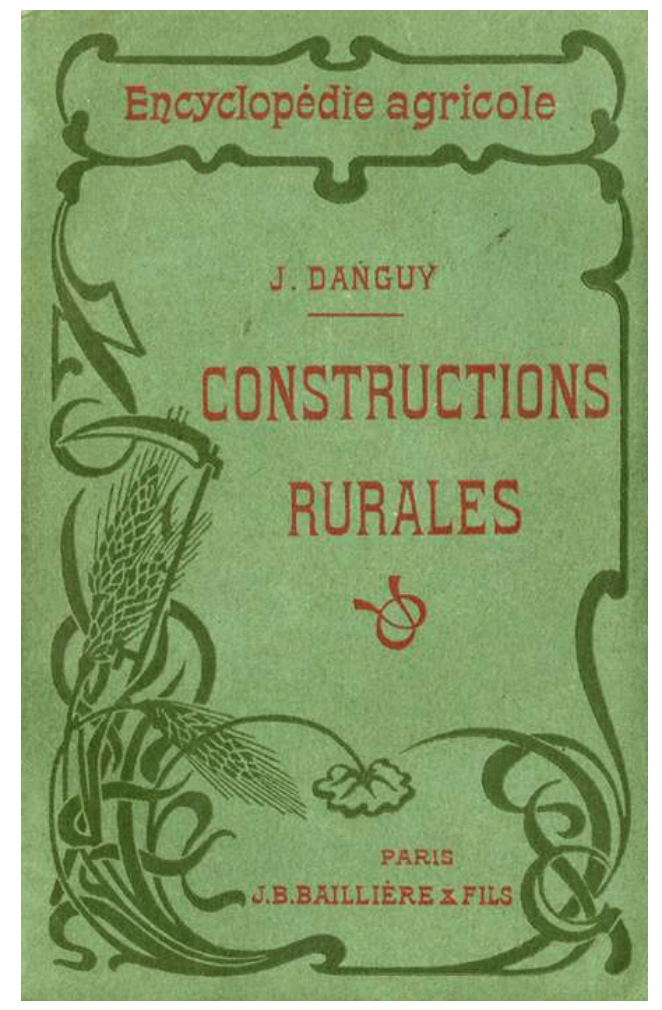

Couverture de l'ouvrage de Jacques Danguy, Constructions rurales, publié dans la collection de l' Encyclopédie agricole sous la direction de G. Wéry, 1908.

Repro. Cappronnier, Jean-Charles, Delorme, Franck. ( ) Jean-Charles Cappronnier, Franck Delorme. 


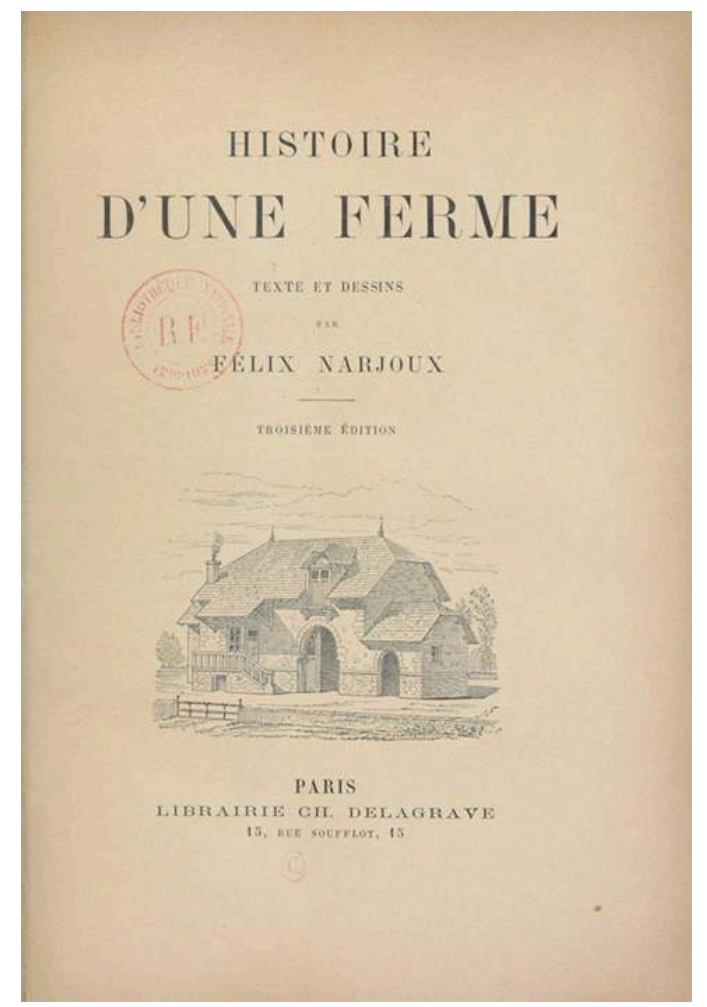

Couverture de l'ouvrage de Félix Narjoux, Histoire d'une ferme. Paris : Librairie Ch. Delagrave, 1882.

Repro. BnF. (c) BnF.

\section{De la maison du cultivateur à la ferme de grande culture : cas d'études et enjeux}

11 Anéanties ou durement touchées par les conflits, les fermes de l'Aisne dans leur diversité de taille et de vocation, sont un vaste champ d'expérimentation tant du point de vue de la rationalisation (dans les grandes exploitations de plateau), du point de vue de l'hygiène (dans les micro-exploitations regroupées en villages) que dans la définition d'une architecture de prestige (grands domaines aristocratiques), et il n'est bien entendu pas exclu que dans certains cas, les divers aspects se retrouvent dans une même ferme. Pour l'étude, le choix s'est porté sur trois types de fermes aux caractères fonctionnels et architecturaux illustrant, d'une part, deux zones géographiques et géologiques bien différentes - le plateau du Sud-Soissonnais pour les deux premiers, les confins du Vermandois pour le troisième - et, d'autre part, plusieurs configurations socio-économiques, de la micro-exploitation familiale, à l'exploitation quasi industrielle relevant d'un grand domaine châtelain. On a délibérément exclu les cas pouvant appartenir à des territoires demeurés de manière durable en zone rouge (chemin des Dames) et, de ce fait, soumis à un régime particulier en vue de leur reconstitution foncière. Les exemples retenus concernent essentiellement des architectes dont les archives sont conservées au Centre d'archives d'architecture du $\mathrm{XX}^{\mathrm{e}}$ siècle de la Cité de l'architecture et du patrimoine mais d'autres fonds d'archives privées d'architectes auraient aussi pu alimenter la recherche. 


\section{Les maisons de cultivateur des villages du Sud-Soissonnais}

donc pas seulement sur la rationalisation des grandes exploitations. Si le remembrement foncier, certes limité, et le transfert des dommages de guerre permettent la concentration de l'appareil productif agricole, la mise en place de normes d'hygiène touche aussi les petites fermes familiales. Dans ce dernier cas, précisément, malgré la modestie du sujet, les architectes contribuent fortement à ces évolutions, notamment en période de reconstruction. Concernant l'apport des architectes aux améliorations de ce type de bâtiments d'échelle somme toute réduite, il convient peut-être au préalable de se référer à ce qu'en écrit Jules Guadet à l'attention des professionnels: «La petite propriété morcelée ne comporte pas ces bâtiments spéciaux: la maison du cultivateur, souvent bien insuffisante, se complète par une petite étable, un grenier, une cabane à porc, une basse-cour, un hangar. »

Nous l'avons vu précédemment, le département de l'Aisne fut durement touché par les destructions engendrées par la guerre de positions, comme de mouvement. La région de Soissons, secteur essentiellement rural, a été dévastée en raison de sa proximité avec la ligne de front. De nombreux architectes ont travaillé pour les diverses coopératives de reconstruction, nous nous intéresserons plus particulièrement à l'un d'entre-deux, Gaston Ernest, né en 1867 et décédé en 1949, qui est un exemple très éclairant de professionnel engagé dans la Première Reconstruction.

Élève de Ginain à l'École des beaux-arts de Paris de 1887 à 1893, ses liens avec le département de l'Aisne sont anciens. En effet, il expose au Salon de 1899 des relevés de l'abbaye de Longpont située près de Soissons, qui sont publiés dans la revue La Construction moderne ${ }^{16}$. En 1893, il est aussi nommé membre de la commission d'hygiène de la Ville de Paris. Architecte des PTT à partir de 1911, il construit des hôtels des postes à Paris et en région parisienne, mais également dans le département de l'Aisne, à Chauny (1925) et Laon (1932) (fig. n²19).

Figure 19

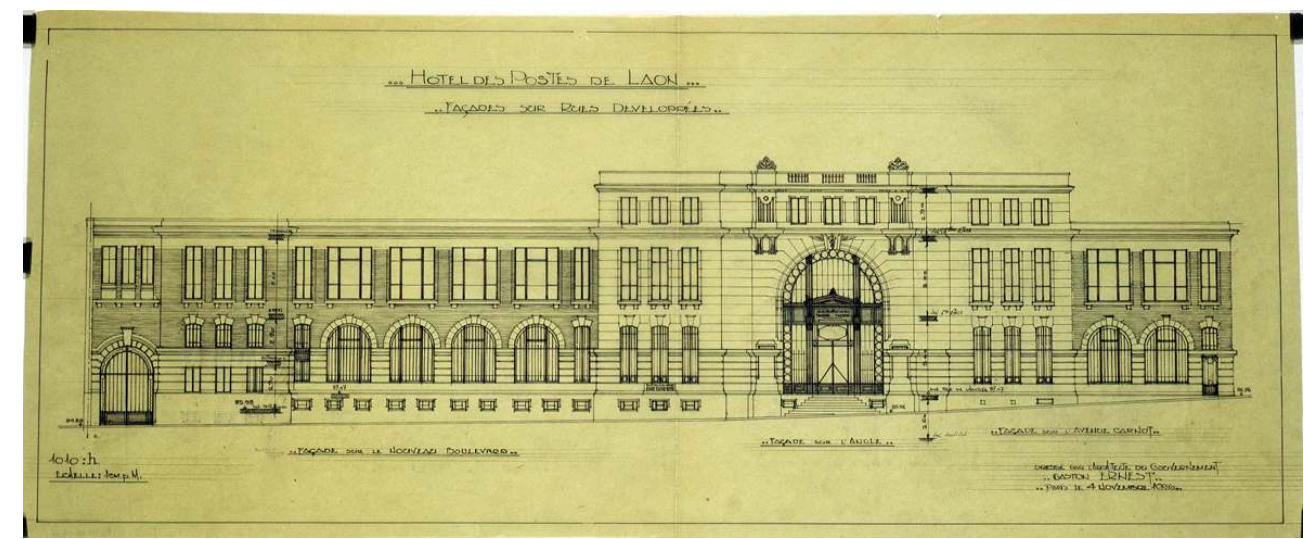

Élévation de l'hôtel des Postes de Laon, par Gaston Ernest, 1931. SIAF, CAPA, fonds Gaston Ernest, 392 IFA 498.

(c) CAPA.

Gaston Ernest s'implique dans la tâche de reconstruction avant tout de manière théorique. Il publie dans le numéro 8 de la revue L'Architecture, un article intitulé 
«Reconstitution des villes dans les régions dévastées ». Il y fait un plaidoyer pour que les architectes occupent un rôle prépondérant dans les procédures de reconstruction détaillées dans la circulaire du 19 février 1919 du ministre des Régions libérées, Albert Lebrun. Le constat des dommages étant le préalable absolu et représentant un effort considérable de la part de l'État. Selon lui, « l'architecte [sous-entendu reconstructeur], présentant des garanties d'honorabilité et de compétence, est donc tout à fait qualifié pour établir seul le constat à défaut d'expert de l'État ».

Lui-même agréé architecte reconstructeur pour l'Aisne et la Marne, Gaston Ernest intervient dans des communes principalement situées de part et d'autre de la limite séparant les deux départements. Dans l'Aisne, il est notamment l'architecte de la Société coopérative de reconstruction de Vierzy et environs. Il deviendra également architecte de la Société coopérative du village de Parcy-et-Tigny. «Cette coopérative avait en effet messieurs Cavelle et Deleour comme architectes. Ayant décidé de liquider leur coopérative et de s'affilier à la coopérative de Vierzy, [il s'est] trouvé l'architecte des sinistrés de Parcy-et-Tigny." Cette commune est faite en réalité de deux groupements d'habitations distants d'un kilomètre l'un de l'autre, les villages de Parcy et de Tigny. Nous allons plus particulièrement nous intéresser à des exemples situés à Tigny dont Gaston Ernest reconstruit également la mairie-école (fig. $\left.\mathbf{n}^{\circ} \mathbf{2 0}\right)$.

Figure 20

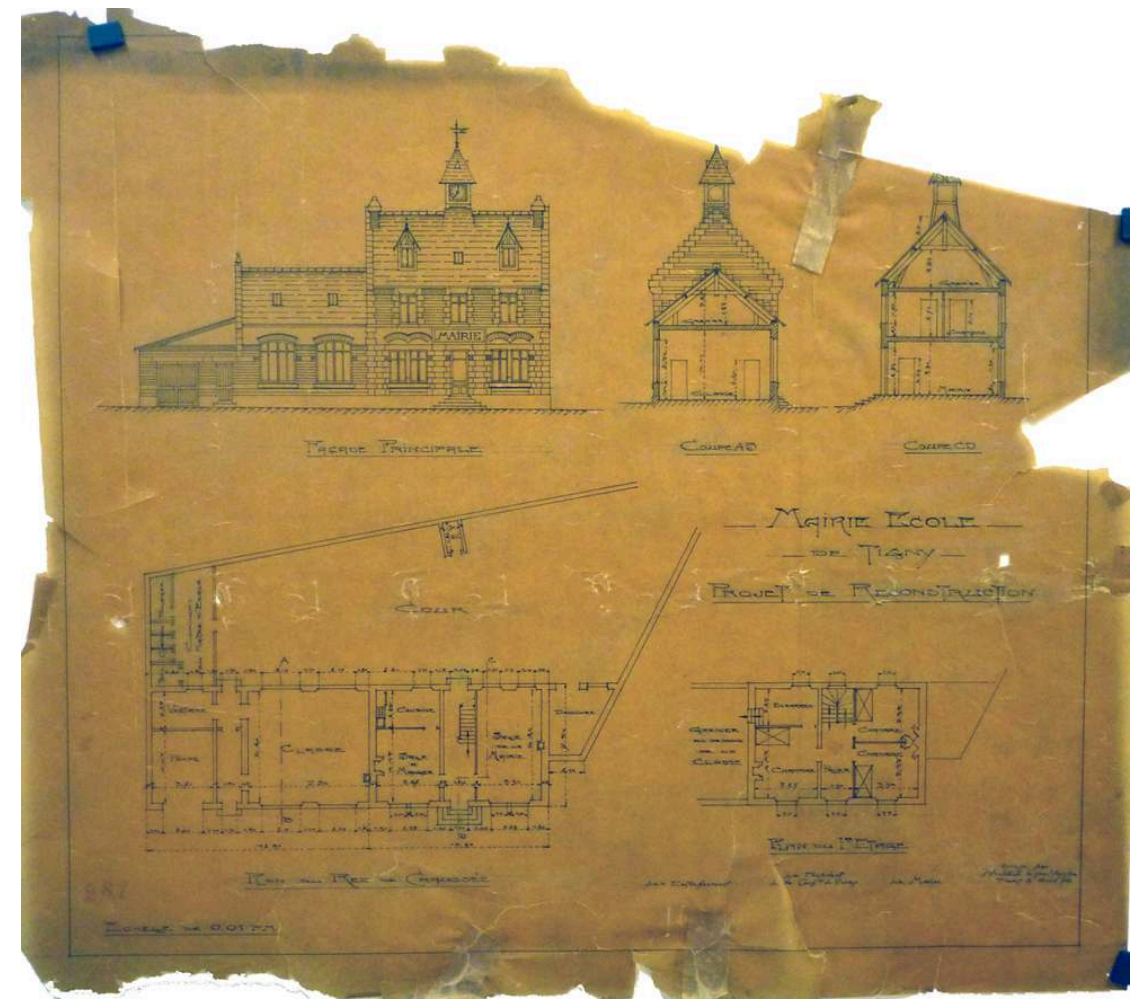

Projet de reconstruction de la mairie-école de Tigny, par Gaston Ernest, 1922. SIAF, CAPA, fonds Gaston Ernest, 392 IFA 212.

(c) CAPA.

Gaston Ernest intervient d'abord d'une manière globale en élaborant les plans d'alignement de Parcy et de Tigny. Puis, il effectue le travail d'évaluation des dommages en décrivant le plus précisément possible les états anciens des biens 
disparus ou partiellement détruits. Enfin, il dresse pour chaque sinistré un projet de reconstruction. Nous sommes ici en présence de territoires du Sud-Soissonnais essentiellement ruraux et constitués d'une majorité de toutes petites exploitations familiales dans lesquelles l'habitation et les locaux agricoles sont étroitement imbriqués. Les enjeux sont nombreux, en premier lieu ceux portant sur l'application de la loi du 15 février 1909 relative à la protection de la santé publique qui impose l'établissement de règlements sanitaires municipaux : «Dans toute commune, le maire est tenu, afin de protéger la santé publique, de déterminer, après avis du conseil municipal et sous forme d'arrêtés municipaux portant règlement sanitaire... » $\mathrm{Ce}$ règlement doit en particulier détailler «les prescriptions destinées à assurer la salubrité des maisons et de leurs dépendances ».

L'application de ces prescriptions dans le travail de Gaston Ernest se déduit de la comparaison des plans des états anciens et des projets de reconstruction. L'imbrication des différents espaces est la caractéristique principale des fermes modestes. C'est à la disparition ou à la réduction de ce phénomène que l'architecte reconstructeur doit s'attacher en priorité. Il faut, là encore, relire Guadet pour trouver une description assez fidèle de la réalité : "Tout cela s'accole à la maison, parfois la pénètre [...]. Ce n'est pas toujours très hygiénique, mais bêtes et gens vivent presque en famille, ce voisinage réciproque défend du froid, et surtout le paysan, très économe par goût, mais aussi par nécessité, n'a pas le moyen de faire autrement. Mais en subissant cette agglomération lorsqu'elle est inévitable, rien n'empêche du moins d'en atténuer les inconvénients par le soin et l'intelligence dans la construction, et par l'observation des règles d'expérience qui ont profondément modifié et modernisé la conception de l'architecture rurale. » 
Figure 21

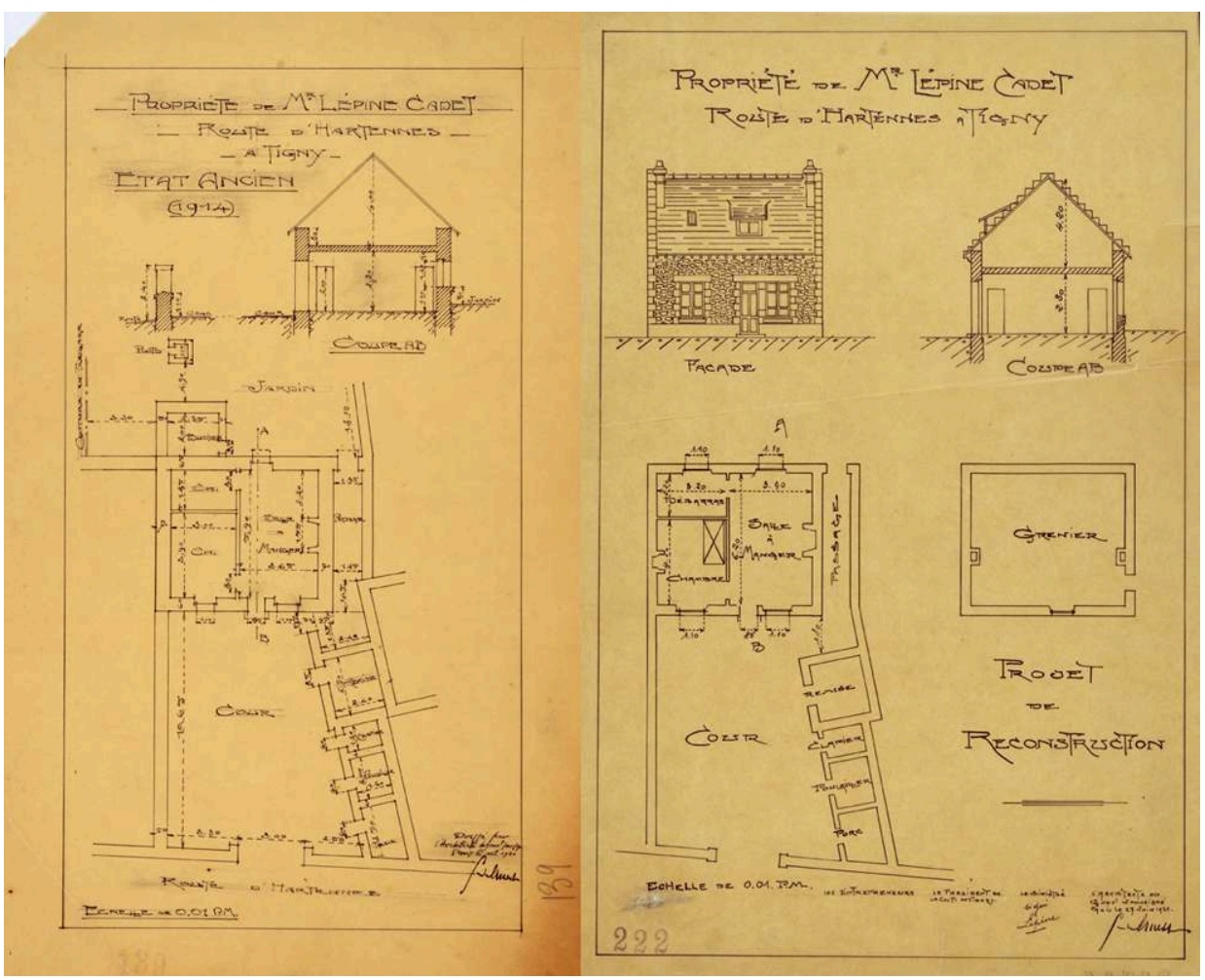

Relevé de l'état ancien (1914) et projet de reconstruction de la propriété de Monsieur Lépine-Cadet à Tigny, par Gaston Ernest, 1920-1921. SIAF, CAPA, fonds Gaston Ernest, 392 IFA 117. (c) CAPA.

Figure 22

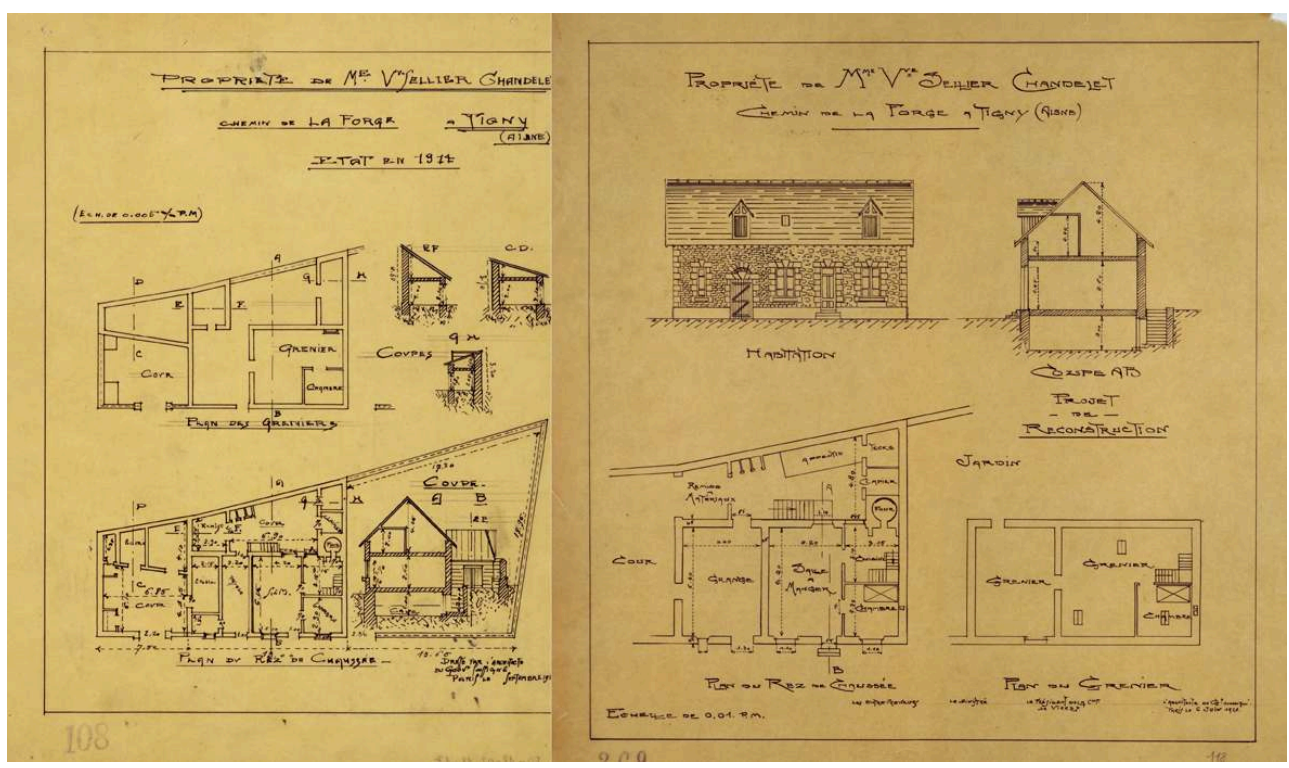

Relevé et projet de reconstruction de la propriété de Madame Sellier-Chandelet à Tigny, par Gaston Ernest, 1920-1921. SIAF, CAPA, fonds Gaston Ernest, 392 IFA 118. (c) CAPA. 
Dans certains cas, il s'agit surtout d'apporter à des constructions enclavées de l'air en facilitant les circulations et les passages, d'une cour à une autre, ou de la cour au jardin (propriété de monsieur Lépine Cadet (fig. $\mathbf{n}^{\circ 21}$ ) ou propriété de madame Sellier Chandelet (fig. $\mathbf{n}^{\circ} \mathbf{2 2}$ )). En même temps, des pièces peuvent être déplacées, regroupées ou supprimées pour des questions d'hygiène ou des exigences fonctionnelles (déplacement de la cuisine sur la cour d'arrivée dans la propriété de madame Nivelle (fig. $\left.\mathbf{n}^{\circ} \mathbf{2 3}\right)$ ). Dans d'autres exemples, c'est la séparation physique entre l'habitation et les locaux agricoles qui est privilégiée (propriété de monsieur Gaulet Pingret (fig. $\left.n^{\circ} 24\right)$ ).

\section{Figure 23}

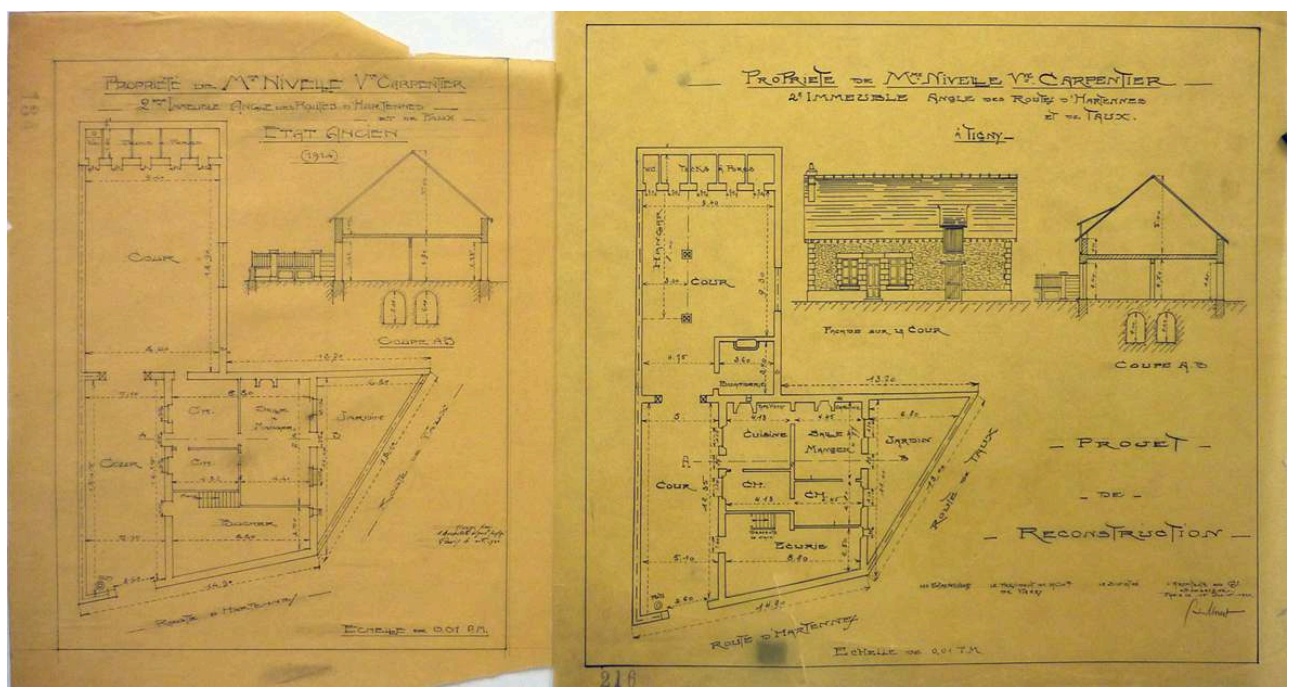

Relevé et projet de reconstruction de la propriété de Madame Nivelle à Tigny, par Gaston Ernest, 1920-1921. SIAF, CAPA, fonds Gaston Ernest, 392 IFA 101.

(C) CAPA. 
Figure 24

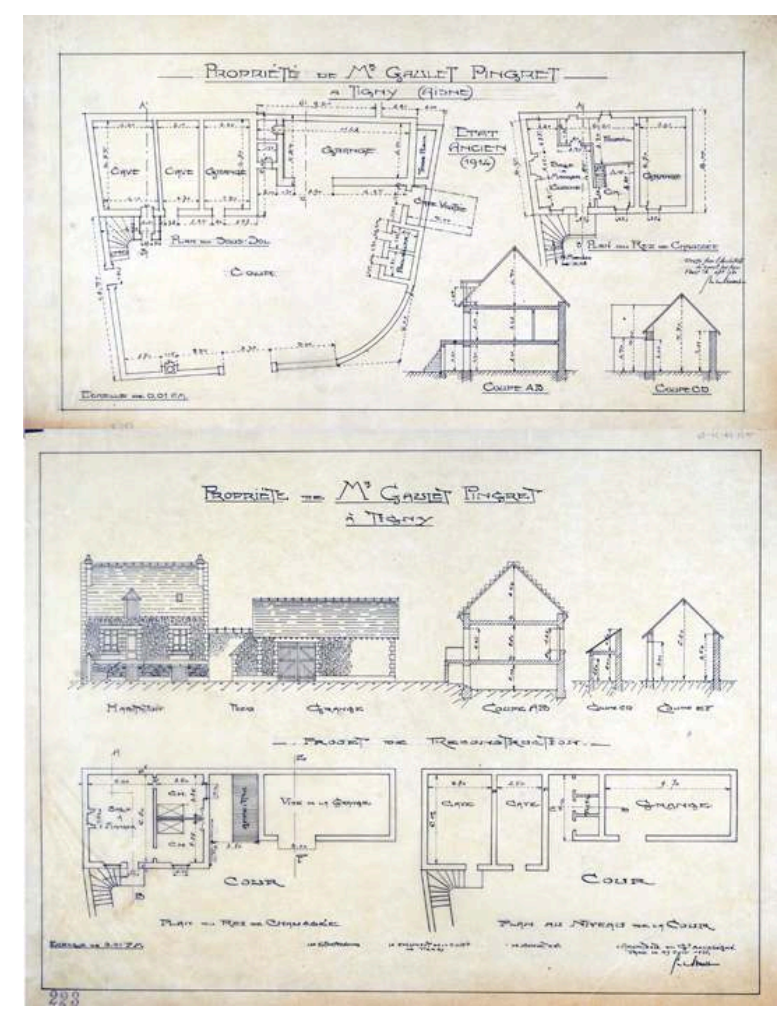

Relevé et projet de reconstruction de la propriété de Monsieur Gaulet-Pingret à Tigny, par Gaston Ernest, 1920-1921. SIAF, CAPA, fonds Gaston Ernest, 392 IFA 105.

(c) CAPA.

Gaston Ernest élabore également des modèles d'éléments types destinés à rationaliser les locaux ou leur aménagement, des éléments qui pourront être produits localement en petites séries puis utilisés au gré des besoins dans les différents projets de reconstruction. Dans les petites fermes familiales, ce sont surtout les locaux annexes qui bénéficient de cette rationalisation, notamment les poulaillers, les clapiers ou les tecks (pièces pour les porcs) (fig. $\mathbf{n}^{\circ} \mathbf{2 5}$ ), qui font l'objet d'une systématisation. Dans le cas des villages de Parcy-et-Tigny, ce sont les modèles produits pour la commune de Bucy-le-Long sur laquelle intervient aussi Ernest, qui seront employés. 
Figure 25

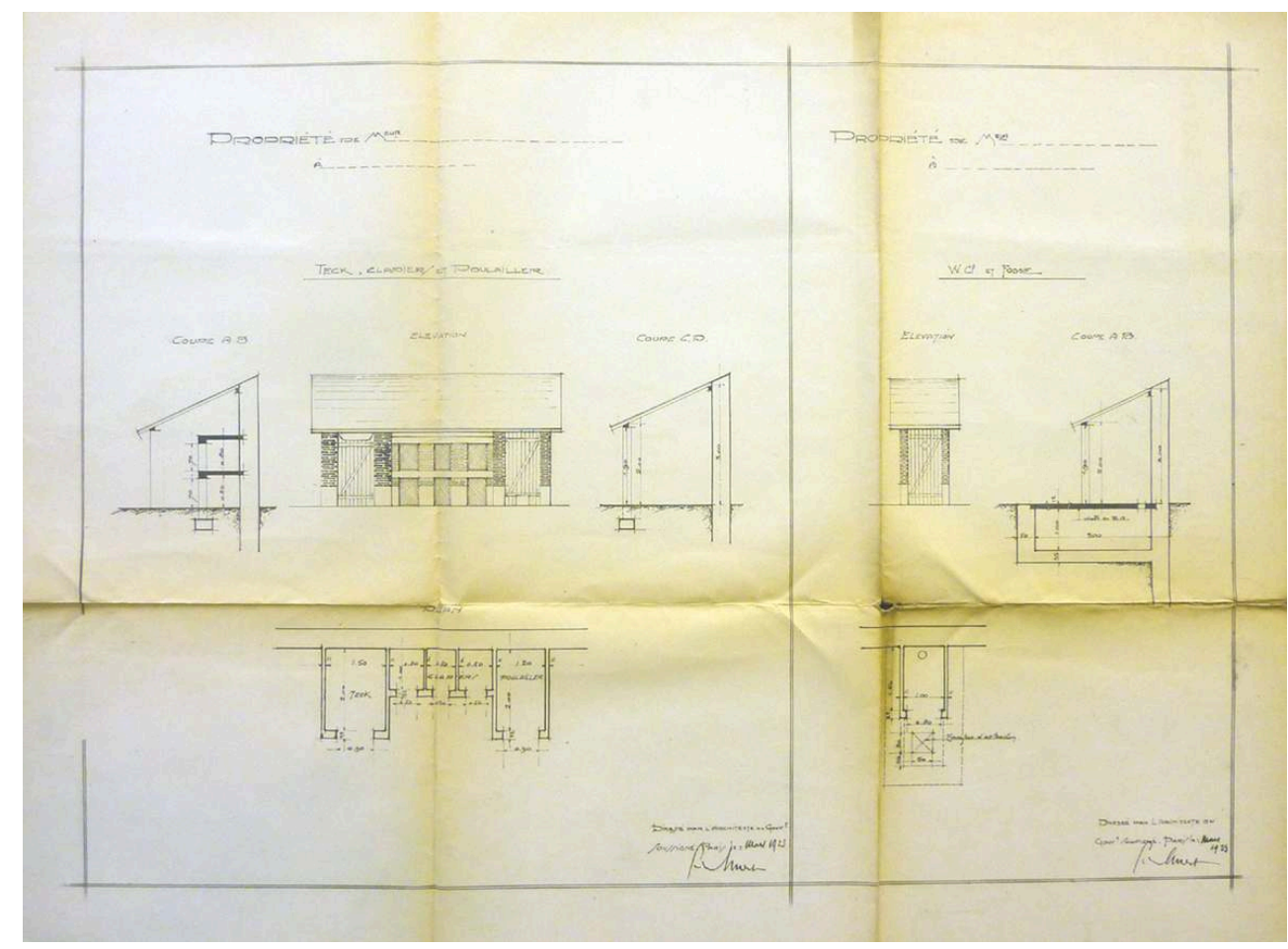

Plan-type de locaux annexes pour la coopérative de Bucy-le-Long, par Gaston Ernest. SIAF, CAPA, fonds Gaston Ernest, 392 IFA 453.

(c) CAPA.

\section{Les fermes de moyenne et grande culture}

21 Plus l'exploitation gagne en taille, plus les améliorations introduites sont nombreuses et significatives. La plupart de celles-ci porte sur les bâtiments agricoles eux-mêmes, sur leur implantation les uns par rapport aux autres ou sur leur plan, mais assez peu sur l'habitation, qui est souvent déjà avant destruction un édifice en soit séparé des autres corps de bâtiment. Dans le tome 3 de son ouvrage Murs et Toits pour pays de chez nous, Charles Letrosne donne la description suivante : «En général, la ferme de grande culture, établie dans le Soissonnais, donnera l'aspect d'une grange avec différents bâtiments placés par étages, les bergeries étant logées dans les anciennes carrières ${ }^{17}$. " 


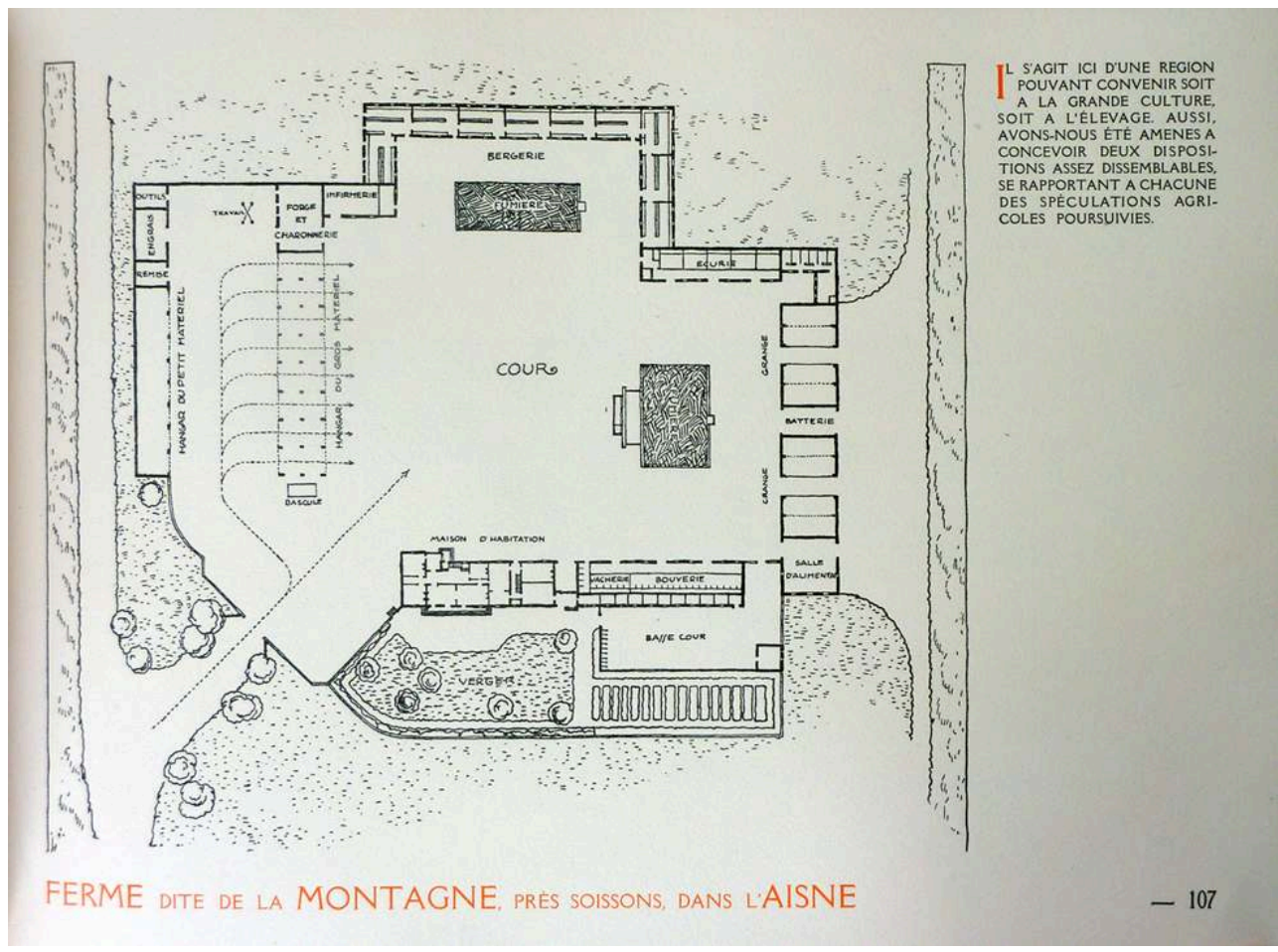

Plan d'ensemble de la ferme dite de la Montagne à Bucy-le-Long, publié par Charles Letrosne dans le tome 3 de Murs et toits pour pays de chez nous, 1926.

Repro. Cappronnier, Jean-Charles, Delorme, Franck. (c) Jean-Charles Cappronnier, Franck Delorme.

Comme exemple, il présente une ferme, située à Bucy-le-Long, dont il est probablement l'auteur (fig. $\mathbf{n}^{\circ}$ 26). Le cas de cette ferme dite « de la Montagne » est particulièrement intéressant pour notre étude car, outre le fait qu'elle se trouve dans la même aire géographique, elle est issue elle aussi de la Reconstruction. « Une exploitation agricole dans le Soissonnais comporte un matériel abondant: charrues Brabant double, scarificateurs, herses, rouleaux, bineuses, moissonneuses-lieuses, arracheuses de betteraves, rouleaux à cheval, faucheuses, etc. Tout ce matériel sera placé sous un hangar vaste et haut, avec des accessoires nombreux qui comprendront fort judicieusement une forge destinée aux réparations. Sous ce même hangar seront placés le travail à ferrer les bœufs, la réserve des engrais et le parc du petit matériel. Notre plan est donc adapté très rationnellement à la culture intensive du sol, qui nécessite en ces régions fertiles, un puissant capital d'exploitation. » Cette description pourrait tout aussi bien s'appliquer à un exemple similaire pris dans la production de Gaston Ernest à Tigny. 
Figure 27

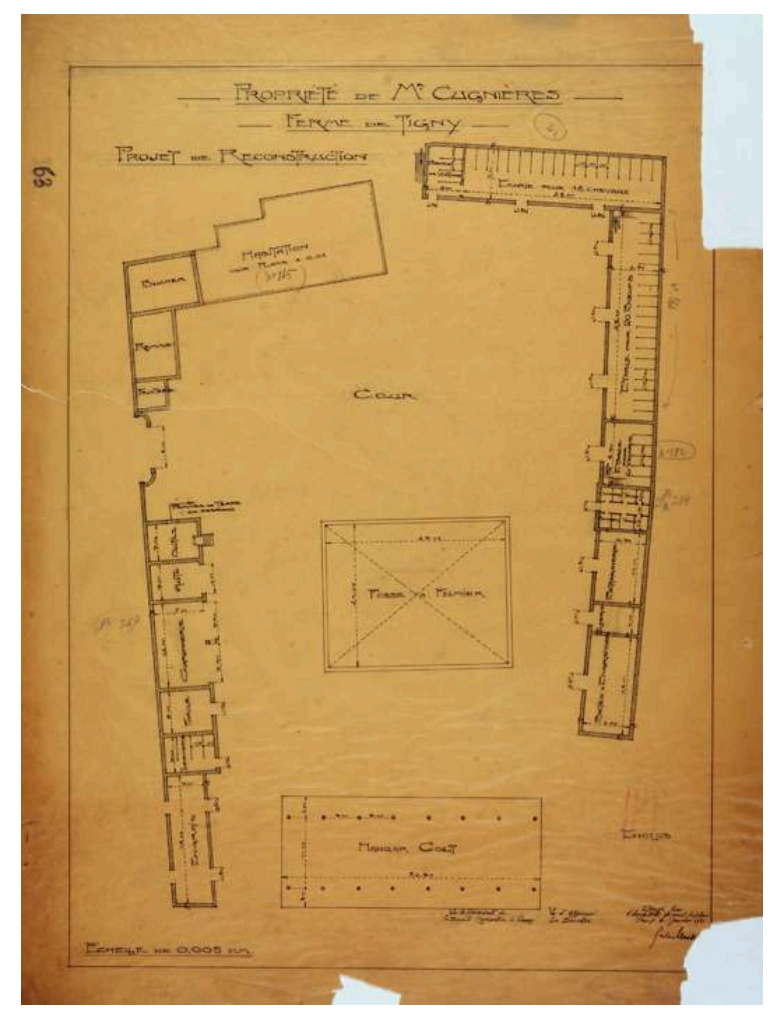

Plan d'ensemble de la ferme de Monsieur Cugnières (1921) à Parcy-et-Tigny, par Gaston Ernest, 1921. SIAF, CAPA, fonds Gaston Ernest, 392 IFA 123.

(C) CAPA.

Il s'agit d'une exploitation agricole pour monsieur Cugnières à Tigny (fig. $\left.\mathbf{n}^{\circ} \mathbf{2 7}\right)$ dont les plans de reconstruction sont datés de 1921. Comme dans la ferme présentée par Letrosne, les bâtiments d'exploitation se répartissent autour d'une cour. Face à l'accès principal se trouvent les étables pour bovins et, en retour d'équerre, les écuries pour chevaux. De l'autre côté de la cour, le long du chemin d'accès, se succèdent les ateliers et les réserves. Le petit côté est fermé par le hangar à machines agricoles. Les élévations des différents bâtiments sont composées de manière régulière mais sans symétrie, les ouvertures et fermetures étant adaptées à la destination des locaux (fig. $\mathbf{n}^{\circ} \mathbf{2 8}$ ). Les matériaux sont choisis en conséquence : murs en maçonnerie de moellon enduit ou non, chaînages et encadrements en brique (conformément à l'usage local), linteaux métalliques de portes cochères. L'habitation du fermier est implantée suivant les prescriptions des traités, dans une position centrale mais avec vue sur le portail d'accès principal. Elle seule possède un caractère bourgeois, presque savant, mais ne comporte malgré tout qu'un rez-de-chaussée (fig. $\mathbf{n}^{\circ} \mathbf{2 9}$ ). 
Figure 28

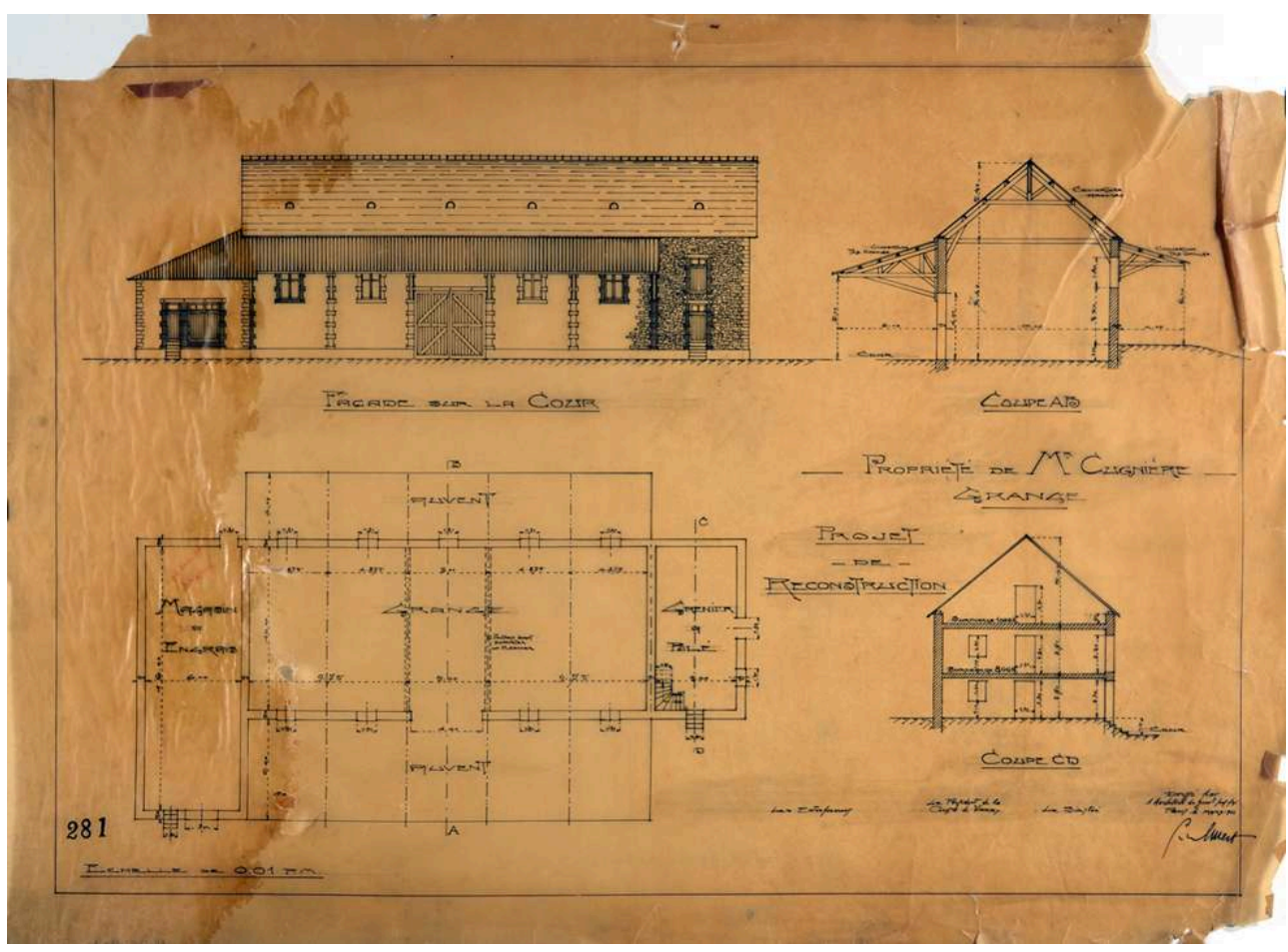

Plans et élévation du projet de grange de la ferme de Monsieur Cugnières (1921) à Parcy-et-Tigny, par Gaston Ernest, 1921. SIAF, CAPA, fonds Gaston Ernest, 392 IFA 123.

(c) CAPA.

\section{Figure 29}

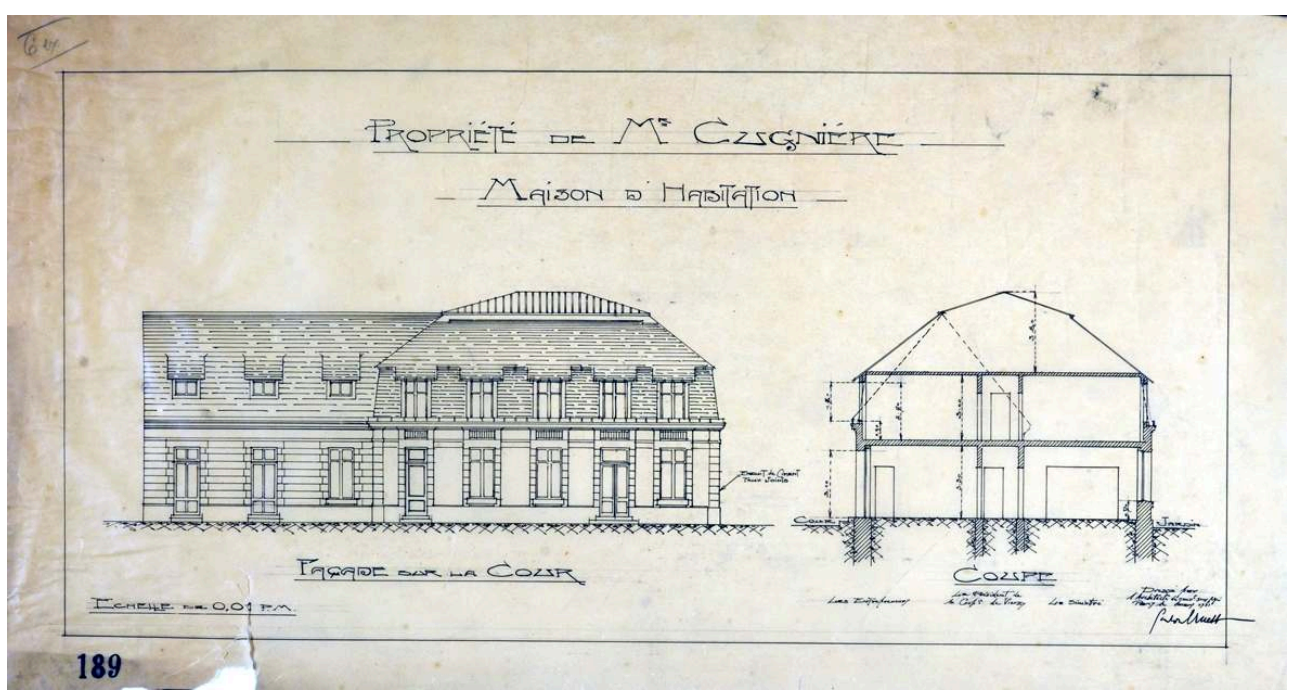

Elévation et coupe de la maison d'habitation de la ferme de Monsieur Cugnières (1921) à Parcy-etTigny, par Gaston Ernest, 1921. SIAF, CAPA, fonds Gaston Ernest, 392 IFA 123. (c) CAPA.

Près de cette première ferme, seulement séparée d'elle par un chemin, Gaston Ernest projette une exploitation dédiée à l'élevage pour monsieur Riant. Essentiellement constituée d'une grande bouverie et d'une grande grange à fourrage, elle est remarquable par certains équipements fonctionnels que l'on y devine, notamment la 
desserte de la bouverie par une voie Decauville, principalement destinée à l'évacuation des fumiers vers la fosse située au centre de la cour, face au bâtiment (fig. $\left.\mathbf{n}^{\circ} \mathbf{3 0}\right)$. Bien que le terrain dessine un polygone irrégulier, une certaine rationalité dans l'implantation des bâtiments se lit; l'habitation du fermier conserve ainsi sa position stratégique, comme dans l'exemple précédent.

Figure 30

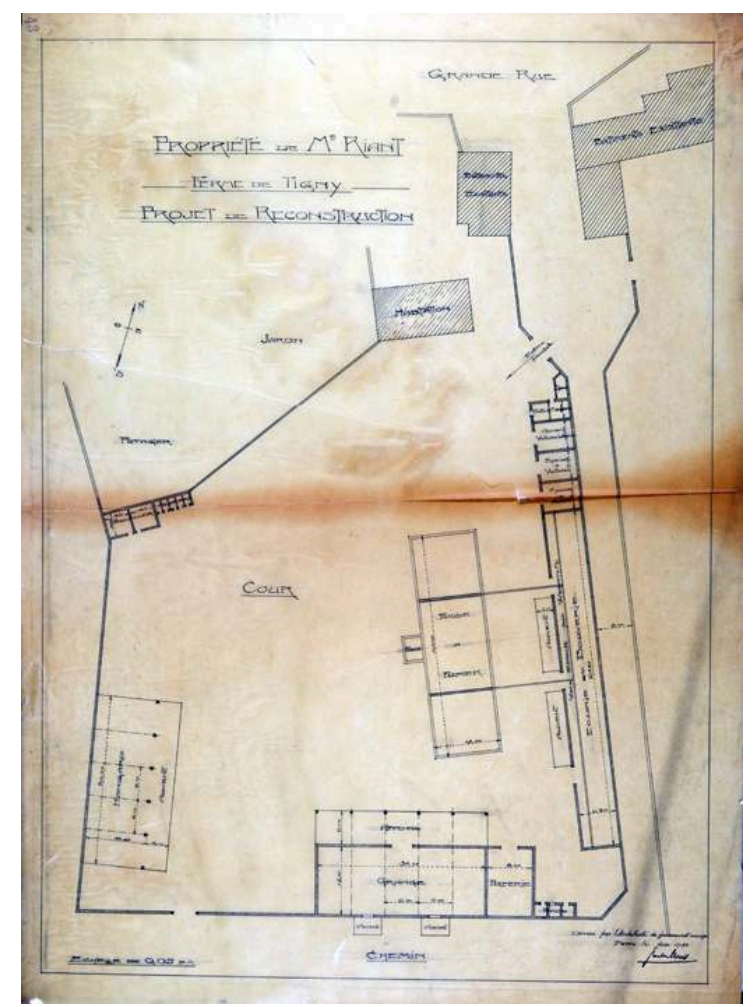

Plan d'ensemble de la ferme de Monsieur Riant (1921) à Parcy-et-Tigny, par Gaston Ernest, 1920-1921. SIAF, CAPA, fonds Gaston Ernest, 392 IFA 119.

(c) CAPA.

\section{Un domaine rural : la ferme de Frières à Frières-Faillouël}

Le troisième cas de ferme étudiée s'inscrit dans le sillage de l'exploitation de grands domaines fonciers d'origine aristocratique ayant traversé sans trop d'encombres les bouleversements de l'époque révolutionnaire et du XIX ${ }^{\mathrm{e}}$ siècle. Depuis le XVIII ${ }^{\mathrm{e}}$ siècle, la famille Le Scellier de Chézelles possède et administre un riche domaine foncier et forestier à Frières-Faillouël. Le déclenchement de la Grande Guerre détruit l'équilibre familial, puisque, à dix jours de distance, le vicomte de Chézelles meurt tragiquement, fusillé par les Allemands pour espionnage, et que son fils, âgé de 21 ans, est tué devant Péronne. Désormais seule, la vicomtesse de Chézelles confie, dès 1920, à l'architecte parisien André Granet (1881-1974) (fig. $\mathbf{n}^{\circ 31}$ ) le soin de relever le domaine dévasté. On peut supposer que des relations de clientèle préexistaient entre la propriétaire sinistrée, qui possédait une résidence à Paris, et l'architecte. En effet, André Granet ne semble pas avoir été, au contraire de Gaston Ernest, désigné comme architecte de coopérative de reconstruction locale et, par ailleurs, un autre de ses principaux clients dans l'Aisne, le sieur Quequignon, qui le charge de reconstruire une grande ferme à 
Villers-Saint-Christophe, lui avait déjà confié des travaux à Paris avant 1914. La commande de constructions agricoles semble une nouveauté pour Granet, plus familier des immeubles de rapport et des bâtiments industriels.

Figure 31

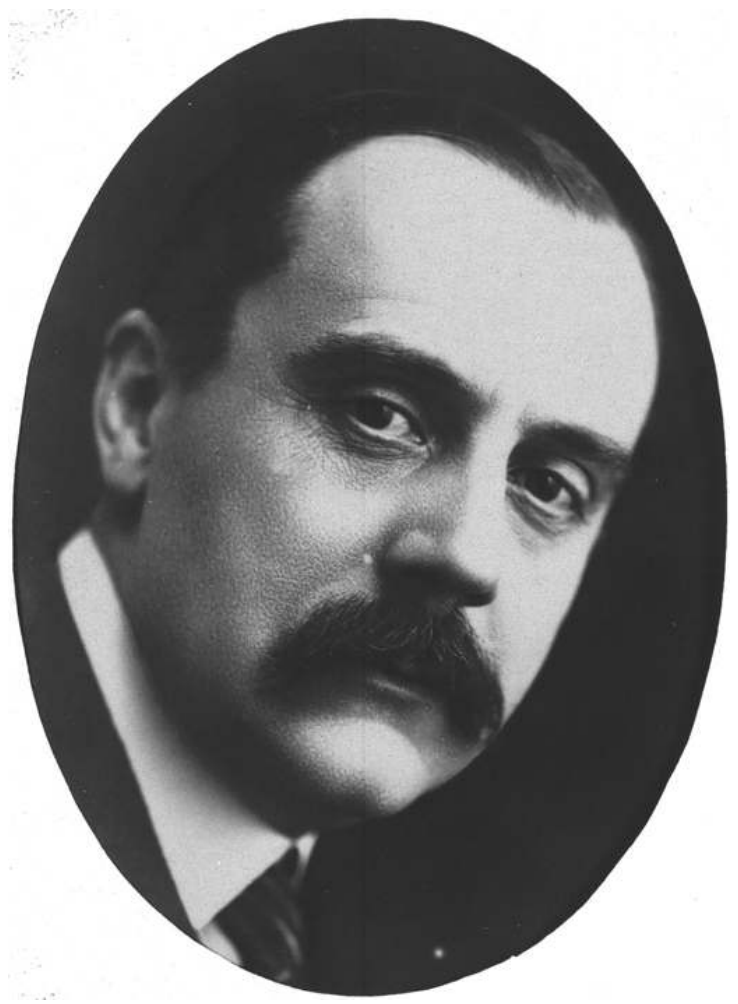

Portrait d'André Granet paru dans Le Journal Lumineux à l'occasion du Salon de l'Aviation de 1926. SIAF, CAPA, fonds André Granet, 86 IFA 430/1.

(c) CAPA.

Né à Paris, fils de l'architecte Antoine Louis Granet et gendre d'Eiffel, diplômé en 1907 (atelier Redon), avant la guerre, André Granet intervient essentiellement dans la capitale. Sa production d'immeubles de rapport de standing est importante et bien connue, comme ceux de la rue Desaix en 1914, ou celui de la rue Lafayette en 1913 (fig. $\mathbf{n}^{\circ} \mathbf{3 2}$ ). Ses réalisations dans le domaine industriel, notamment celui de l'aviation, le sont un peu moins. On lui doit notamment l'aménagement de l'aérodrome de Champagne (1909-1911) et la construction des usines Gnome-et-Rhône à Gennevilliers (1916-1921) (fig. $\mathbf{n}^{\circ}$ 33). Il attacha son nom aux nombreux projets de décoration des Salons de l'aviation organisés à Paris, au Grand Palais, dès 1909. Ses expériences dans la sphère industrielle, comme la construction de cités ouvrières à Lomme, dans le Nord, en 1906-1913, ne furent sans doute pas inutiles à Granet lorsqu'il dut intervenir dans le paysage architectural sinistré des fermes du Vermandois. 
Figure 32

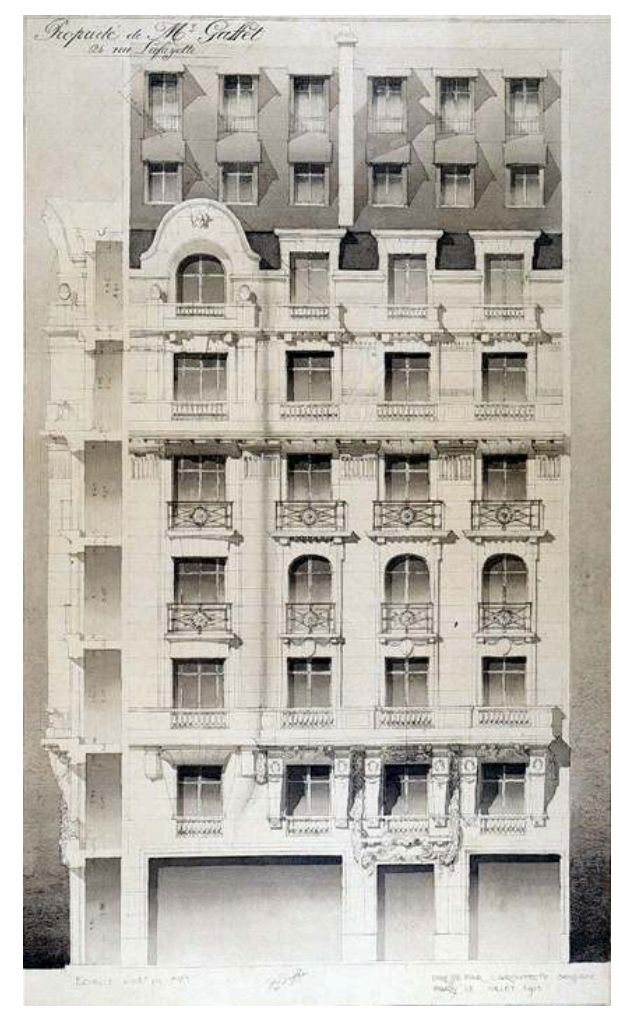

Projet pour un immeuble de rapport, rue Lafayette, par André Granet, 1913. SIAF, CAPA, fonds André Granet, 86 IFA 2012.

(c) CAPA.

Figure 33

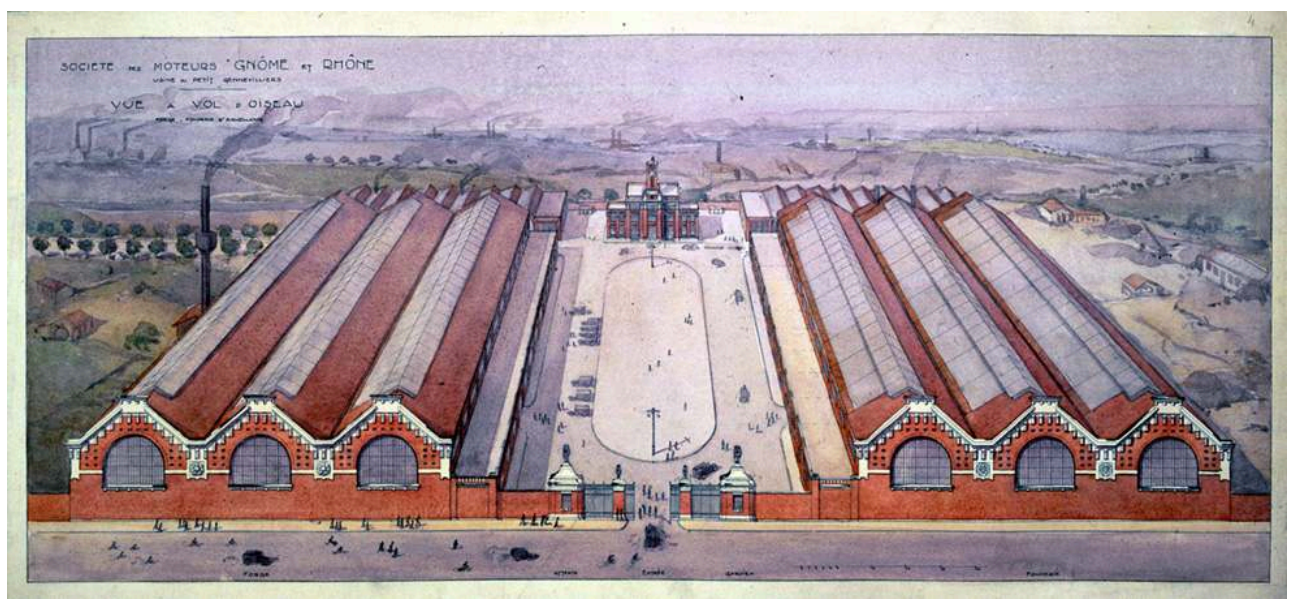

Perspective des usines Gnome-et-Rhône à Gennevilliers, par André Granet, SIAF, CAPA, fonds André Granet, 86 IFA 2015/2.

(c) CAPA.

En 1914, la famille de Chézelles disposait de deux châteaux à Frières-Faillouël. Le premier, situé à Frières même et dénommé « vieux château » semble pouvoir être daté de la fin du XVIII ${ }^{\mathrm{e}}$ ou de la première moitié du XIX ${ }^{\mathrm{e}}$ siècle. Le second, rebâti après 1870 , selon les canons d'un éclectisme foisonnant, est situé entre les hameaux de Frières et Faillouël (fig. n³4). Ce dernier, occupé par les Allemands entre août 1914 et mars 1917, 
fut dynamité lors de leur retrait, tout comme le vieux château, les communs et les fermes. Le grand château sera reconstruit tardivement, entre 1930 et 1935, non pas selon le projet établi par André Granet (fig. $\mathbf{n}^{\circ}{ }^{35}$ ), mais sur les plans de ses successeurs, Gustave Olive et Joseph Martin, tous deux également architectes parisiens (fig. $\mathbf{n}^{\circ} \mathbf{3 6}$ ).

Figure 34

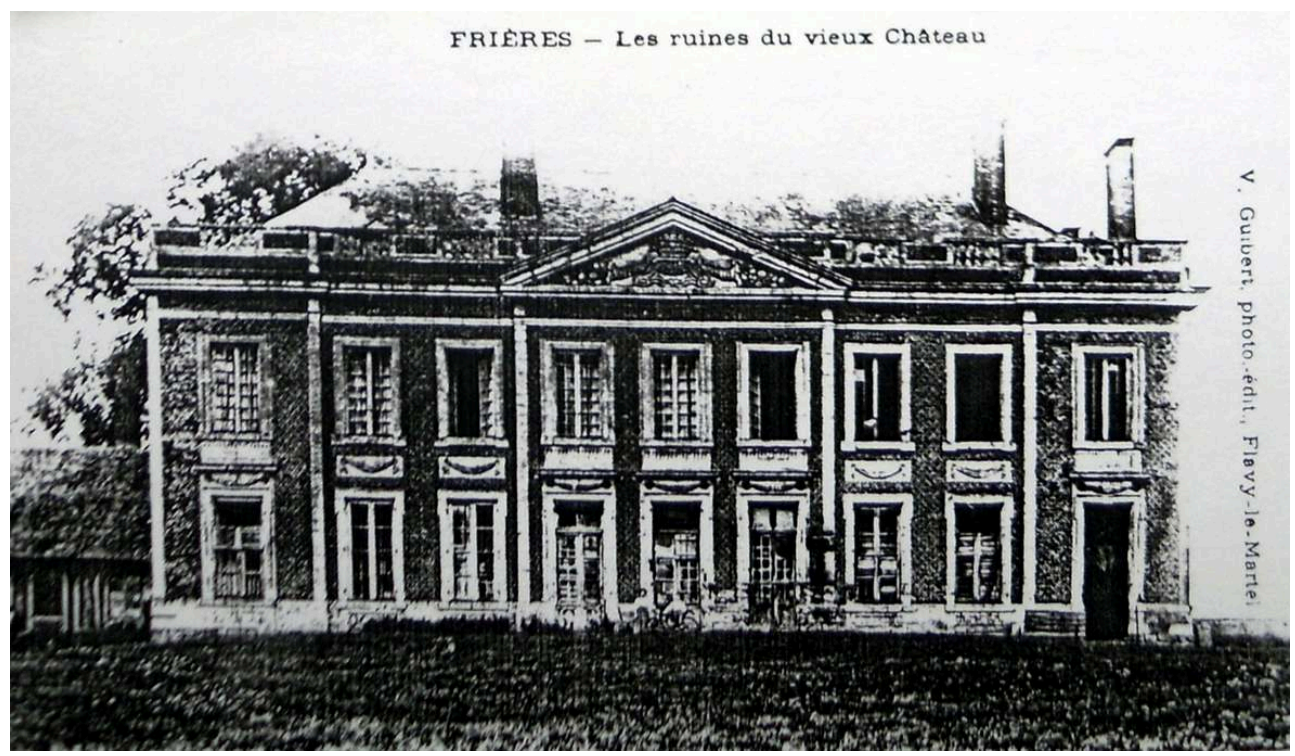

Le vieux château dépendant de la ferme de Frières avant la guerre de 1914-1918.

Carte postale Guilbert, collection particulière.

Figure 35

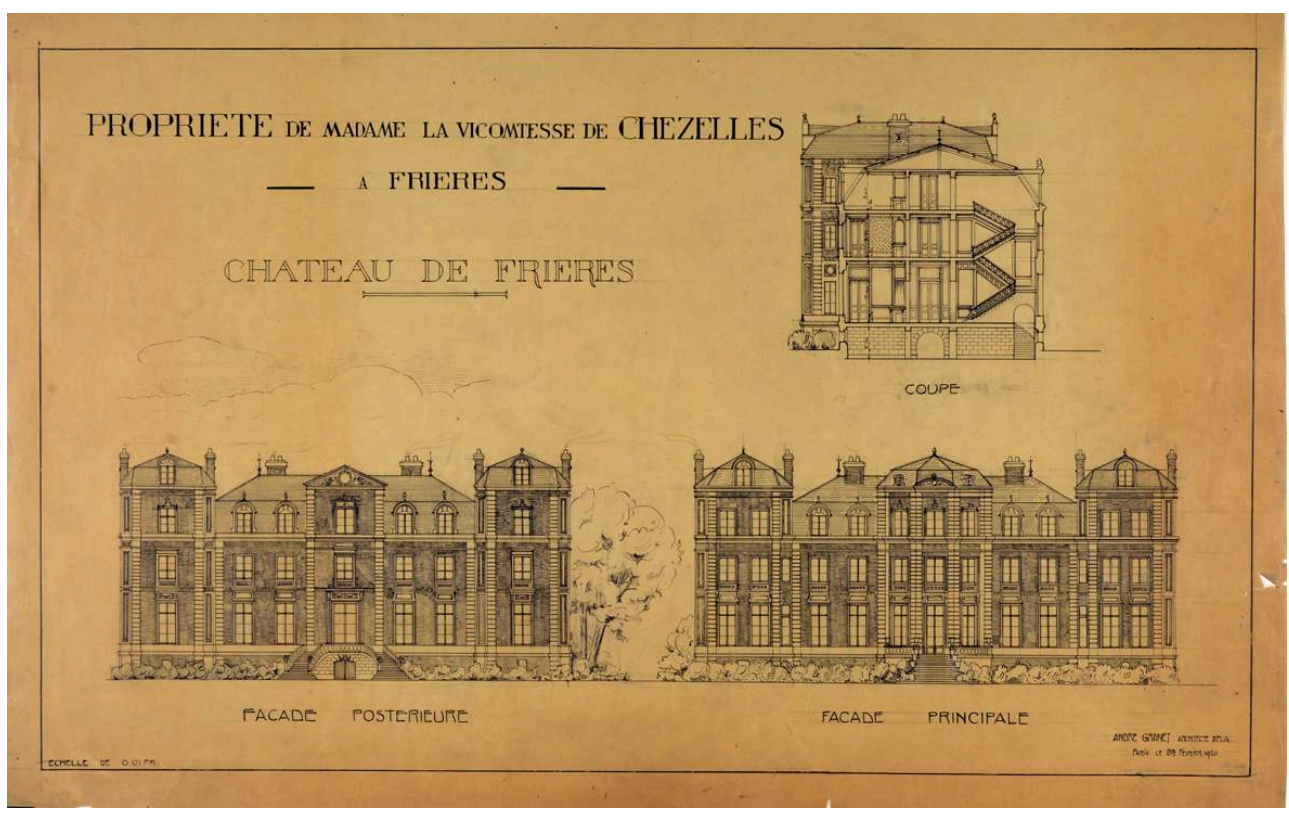

Projet de reconstruction du château de Frières non exécuté, par André Granet. SIAF, CAPA, fonds André Granet, 86 IFA 814.

(c) CAPA. 
Figure 36

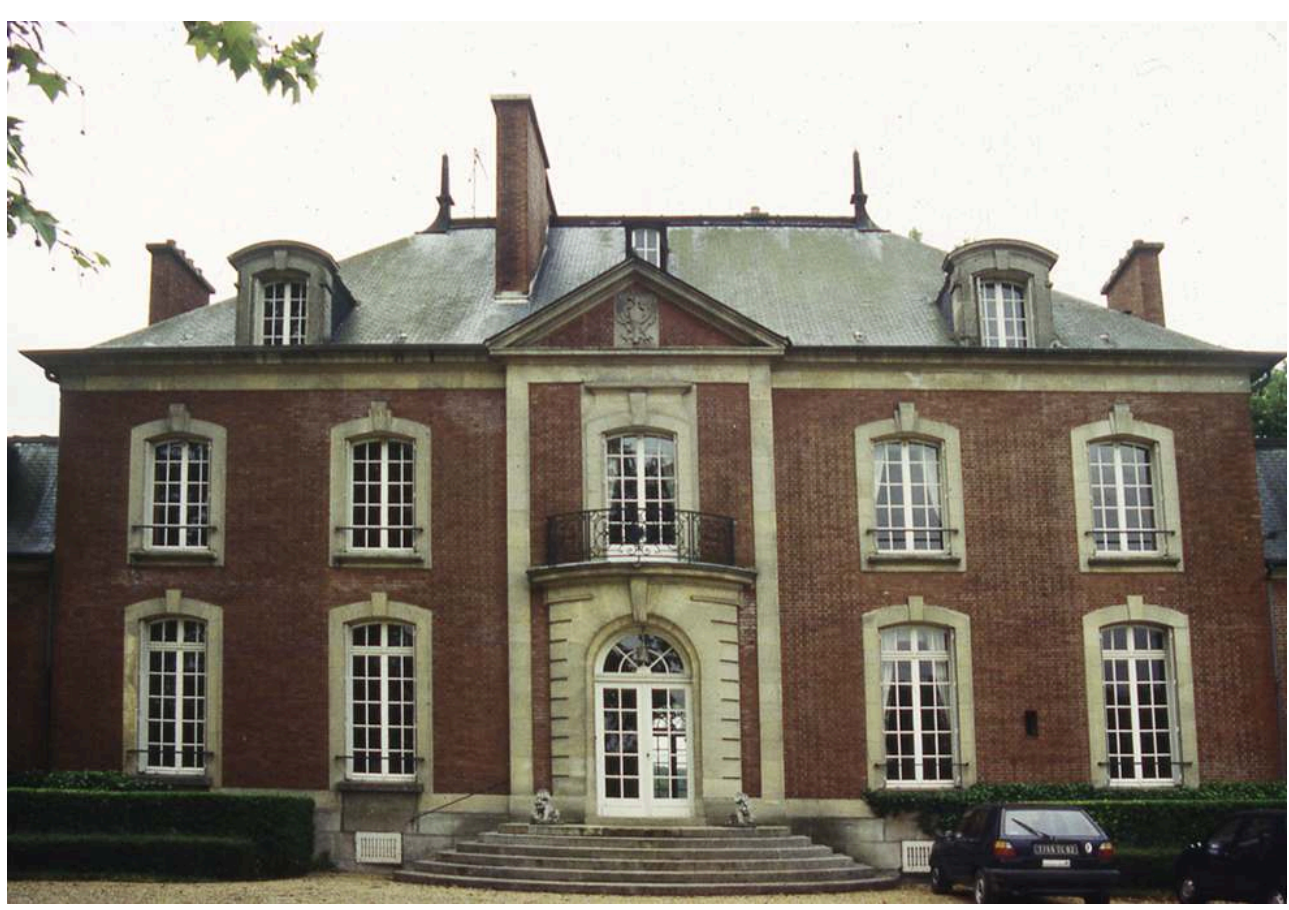

Le château de Frières reconstruit par les architectes Gustave Olive et Joseph Martin.

Phot. Cappronnier, Jean-Charles. (C) Jean-Charles Cappronnier.

Les premières interventions de Granet à Frières sont attestées entre janvier 1920 et octobre 1921, la distinction étant délicate à opérer entre les plans relevant du dossier d'expertise des dommages et ceux relevant spécifiquement du projet de reconstruction. En revanche, le travail opérationnel de Granet semble se limiter aux seules années 1920-1922, les ouvrages de maçonnerie étant exécutés par l'entreprise de travaux publics Douane, Bonhommé et $C^{\text {ie }}$ à Paris. Les ouvrages de charpente et menuiserie, notamment les escaliers des écuries, sont confiés à l'entreprise Huguenin à Paris. Le bâtiment des écuries est doté de planchers métalliques réalisés par la célèbre entreprise parisienne de construction métallique et de ferronnerie d'art Borderel et Robert. 
Figure 37

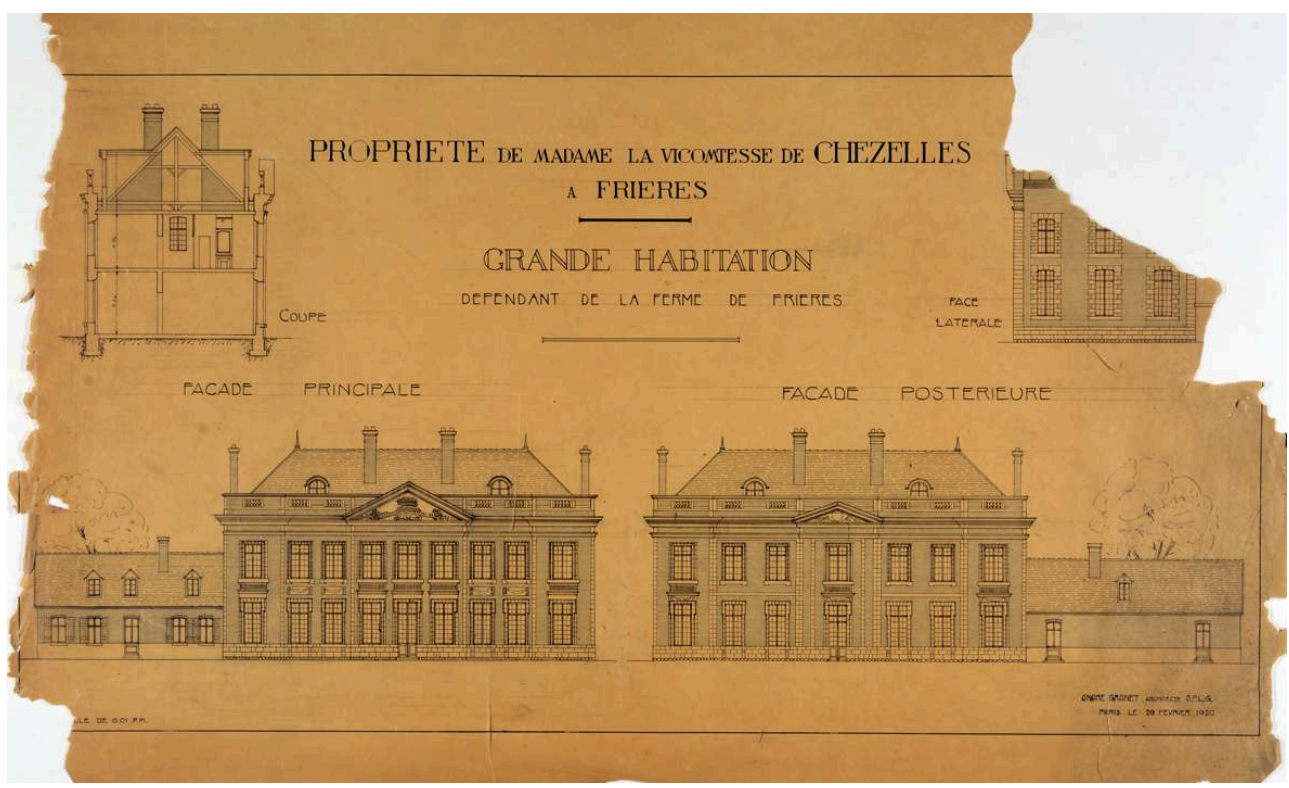

Projet de reconstruction de la « Grande habitation dépendant de la ferme de Frières », par André Granet, 1920. SIAF, CAPA, fonds André Granet, 86 IFA 814.

(c) CAPA.

André Granet établit donc, dès janvier 1920, des plans et élévations du «pavillon d'habitation » destiné à suppléer l'ancien château. En fait, l'élévation se rapporte strictement à une restitution de celui-ci, la désignation proprement châtelaine étant désormais exclue de la terminologie de l'architecte reconstructeur (fig. $\mathbf{n}^{\circ} \mathbf{3 7}$ ). Pour ce qui concerne le domaine châtelain proprement dit, Granet établit également des plans pour une maison de garde-chasse. Les travaux de reconstruction de la grande ferme de Frières, ancienne ferme du « vieux château » prévoient, entre autres, l'édification d'une grange avec appentis, d'une écurie pour douze chevaux avec logement du contremaître, d'un bâtiment abritant laiterie et bouverie, d'une porcherie d'élevage, d'un hangar agricole, de trois hangars à voitures avec grenier à fourrage, enfin le réaménagement du parc à fumier. Le programme possède donc une envergure comparable à celle de l'ancienne exploitation qu'il s'agit alors de remplacer et d'améliorer. 
Figure 38

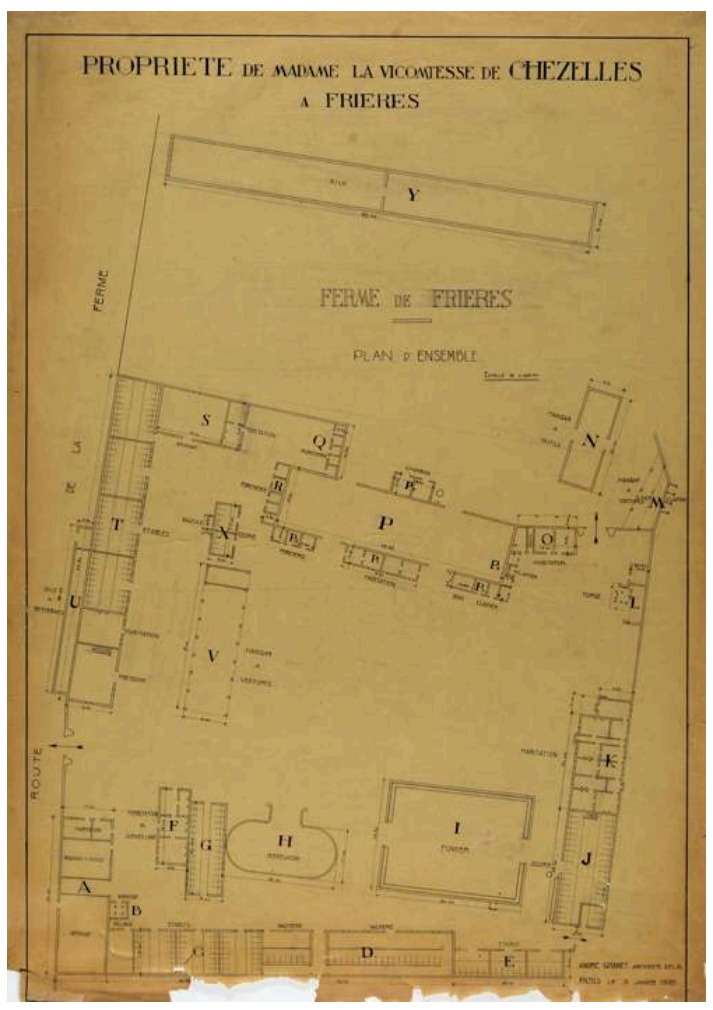

Plan de la ferme de Frières avant 1914, par André Granet. SIAF, CAPA, fonds André Granet, 86 IFA 811. (c) CAPA.

Par rapport au plan de la grande ferme avant 1914 (fig. $\mathbf{n}^{\circ} \mathbf{3 8}$ ), les projets de reconstruction successifs se caractérisent, en premier lieu par une régularisation du plan quadrangulaire des bâtiments et en second lieu, par une concentration de ces derniers, auparavant fortement disséminés (fig. $\mathbf{n}^{\circ} \mathbf{3 9}$ ). Bouverie et habitation du surveillant, jadis implantées non loin de l'abreuvoir, sont ainsi reconstruites le long du chemin d'accès à la ferme. Quant au hangar à voitures, il est déplacé vers le centre de la cour, perpendiculairement à la grande grange. Les bâtiments jointifs abritant les étables et les vacheries et formant l'un des côtés de la cour sont remplacés par un seul et unique bâtiment destiné à accueillir les "bêtes à engrais ». La grande écurie est rapprochée de l'entrée et du hangar à voitures, en lieu et place des étables. Mais la transformation majeure demeure évidemment la suppression de l'habitation du chef d'exploitation, qui fait place à un hangar et à une vacherie, dans l'axe de l'entrée principale. La confrontation avec une vue aérienne actuelle permet de confirmer le processus de régularisation des bâtiments disposés symétriquement autour de la cour, à l'exception de la grange qui, conformément au projet de reconstruction, demeure isolée. Le site du vieux château se discerne parfaitement, et, contrairement au projet de Granet, il n'a pas fait place au pavillon d'habitation, qui devait être relié à la cour de ferme par un passage. L'entrée de ferme, très monumentalisée, a conservé son ancien portail en brique et pierre à fronton triangulaire, qui demeure jalousement et fièrement intégré au projet global de reconstruction (fig. $\left.\mathbf{n}^{\circ} \mathbf{4 0}\right)$. 
Figure 39

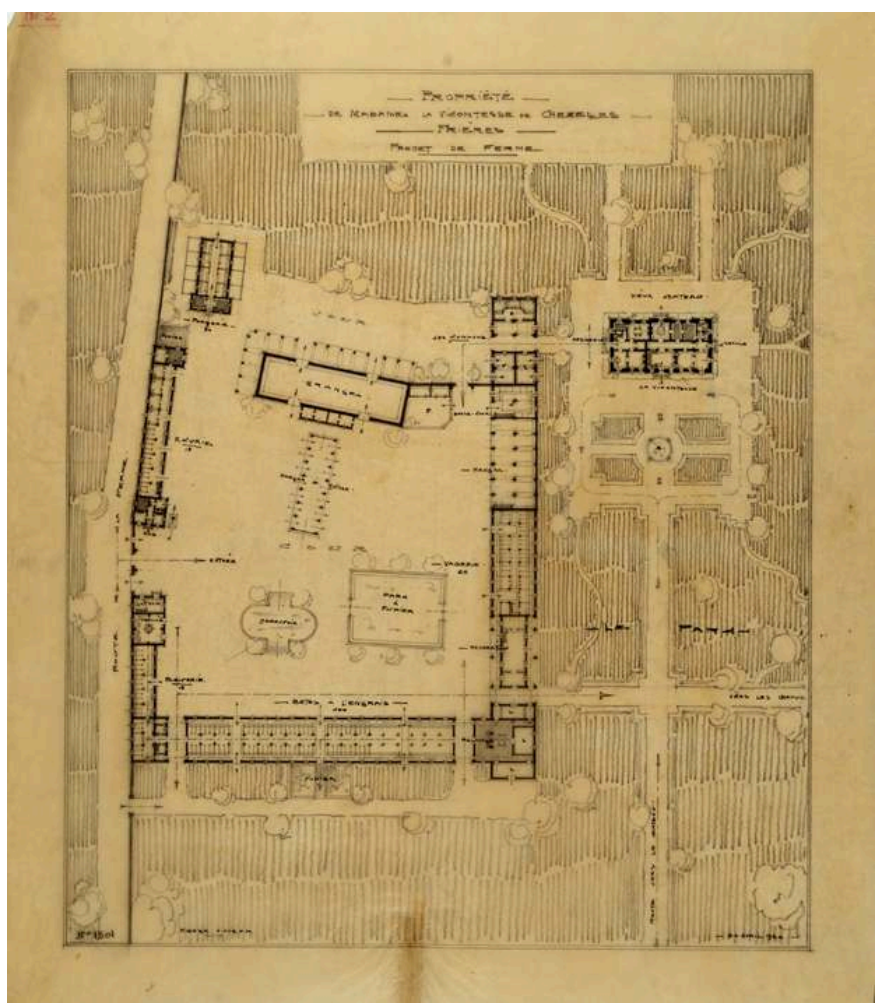

Plan d'ensemble du projet de reconstruction, par André Granet, avril 1920. SIAF, CAPA, fonds André Granet, 86 IFA 811.

(c) CAPA.

Figure 40

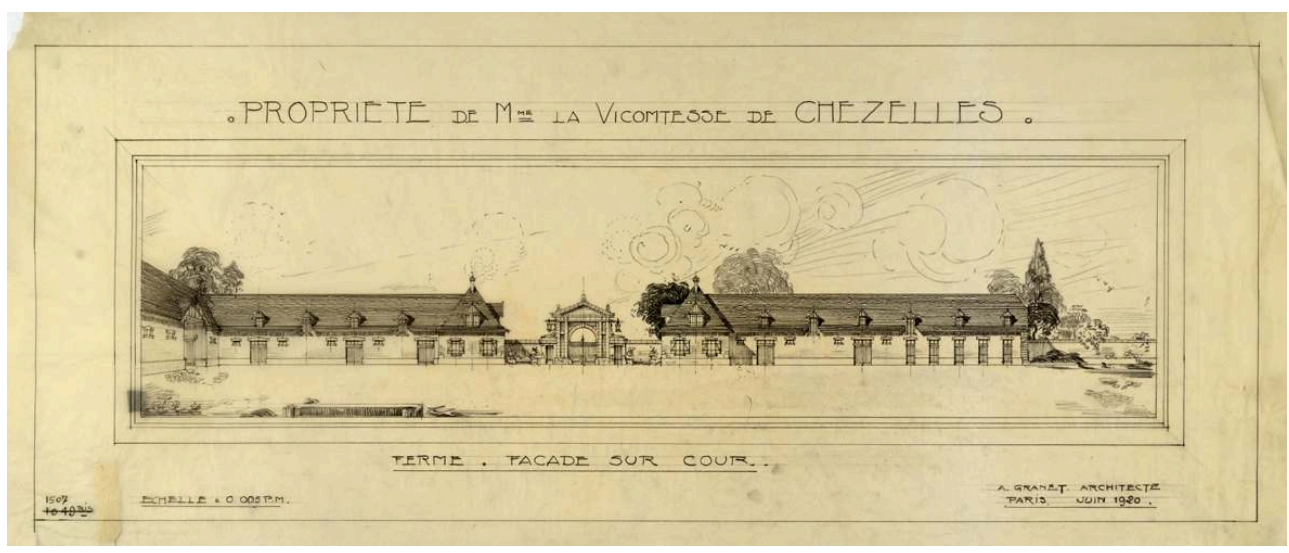

Projet pour l'entrée de la ferme, incorporant un portail monumental ancien, par André Granet, juin 1920. SIAF, CAPA, fonds André Granet, 86 IFA 814.

(c) CAPA.

31 La plupart des bâtiments d'exploitation, notamment ceux réservés à l'élevage (écuries, étables et porcherie) se composent d'un bâtiment de plan rectangulaire, doté d'une couverture à longs pans et croupes, que termine un pavillon de plan carré. Ce principe s'inspire du système régularisé de la ferme en carré, mais il fait aussi référence à des modèles d'architecture classique (fig. $\mathbf{n}^{\circ} \mathbf{4 1}$ ). Le pavillon abrite, tantôt le logement du contremaître dans le prolongement des écuries, tantôt le «logement provisoire » du 
chef d'exploitation à l'entrée de la ferme. À ces logements sont associés des bureaux, en particulier celui, spacieux et orienté vers la cour, du contremaître. Le pavillon abritant le logement provisoire du fermier, doit être lui converti dans un second temps en laiterie et pressoir (fig. $\mathbf{n}^{\circ} \mathbf{4 2}$ ).

Figure 41

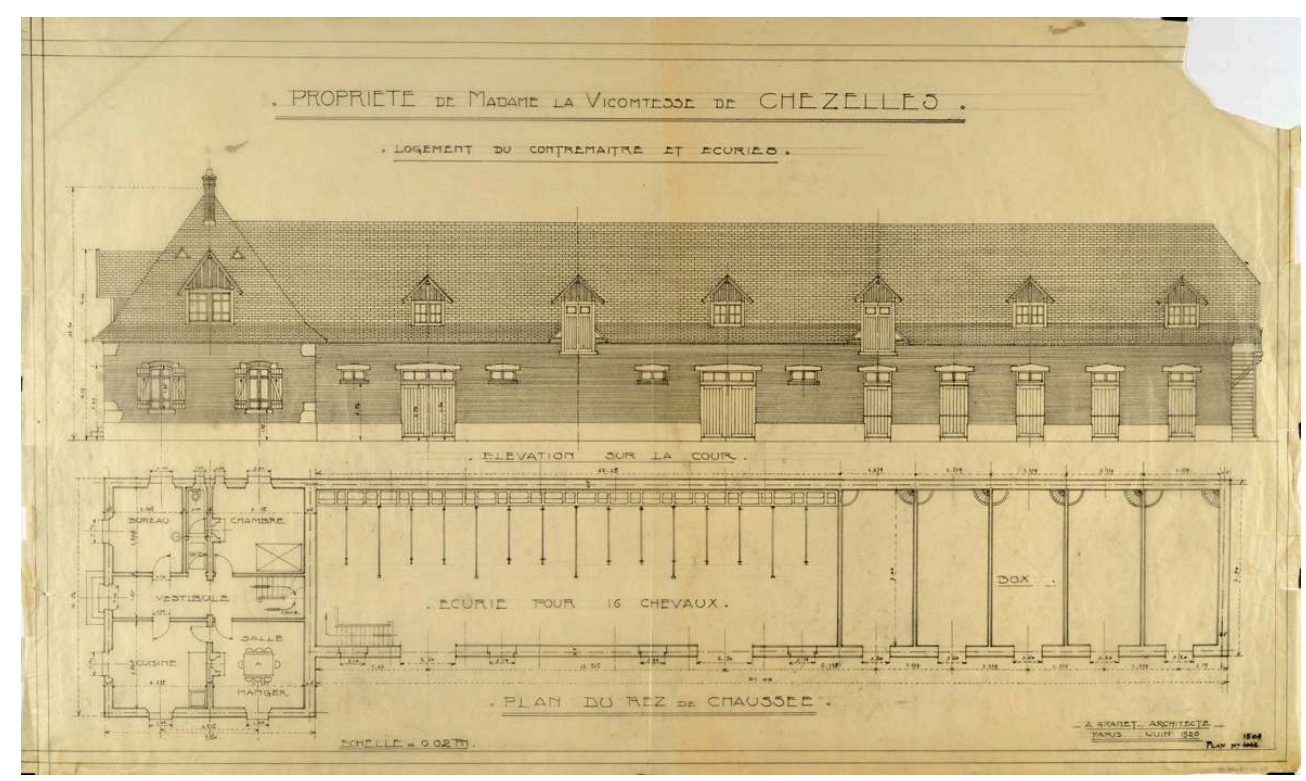

Projet du pavillon du contremaître et du bâtiment des écuries, par André Granet, juin 1920. SIAF, CAPA, fonds André Granet, 86 IFA 815

(c) CAPA. 
Figure 42

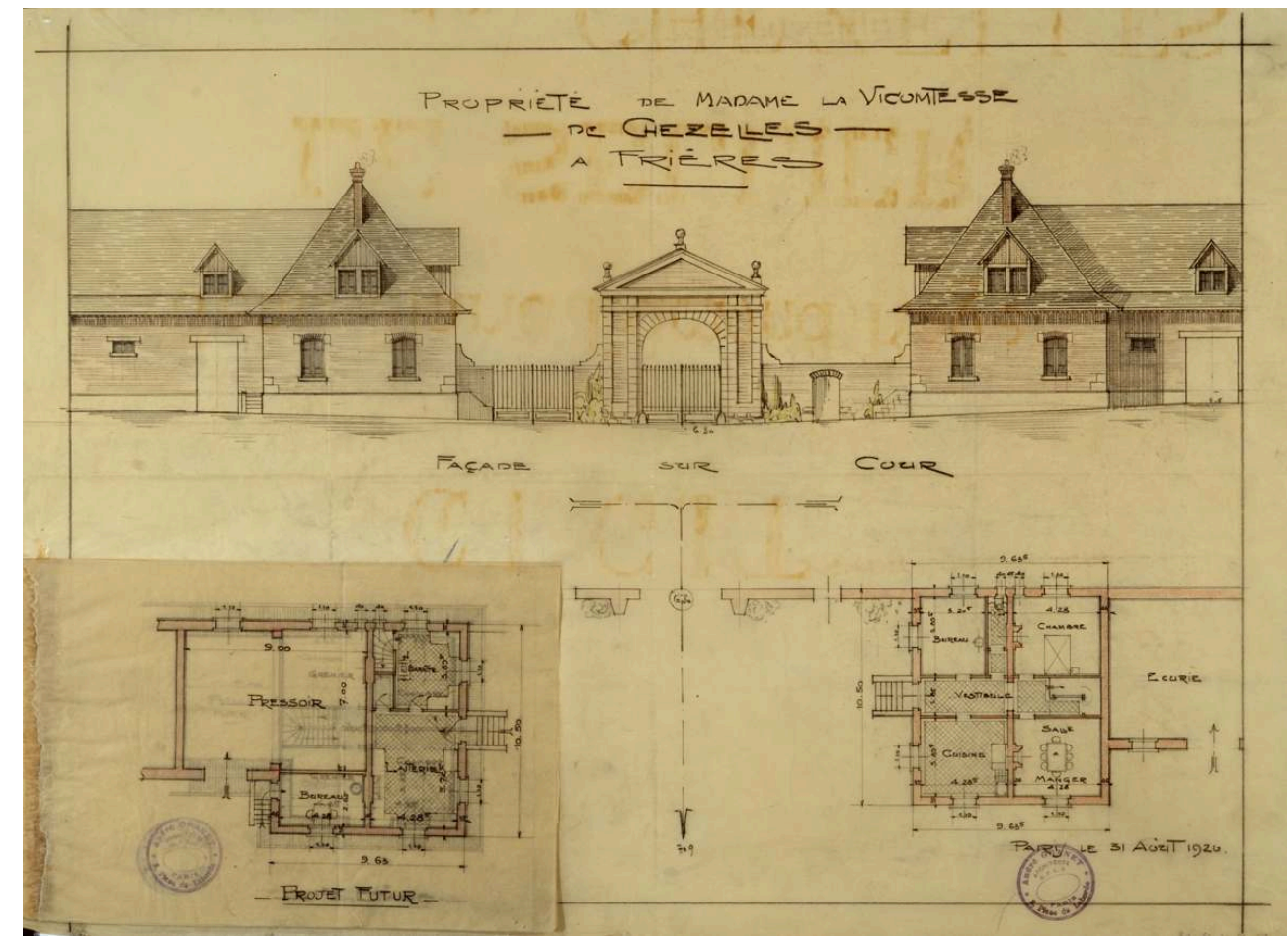

Projet des pavillons de part et d'autre de la grande entrée, par André Granet, août 1921. SIAF, CAPA, fonds André Granet, 86 IFA 814.

(c) CAPA.

Le plan des bâtiments d'exploitation et leurs équipements s'inspirent en grande partie de modèles publiés avant-guerre, en particulier par Danguy. C'est le cas pour la porcherie d'élevage (fig. $\mathbf{n}^{\circ} \mathbf{4 3}$ ) conçue sur le même modèle et formée d'un double rang de loges desservi par un couloir central et flanqué de courettes individuelles latérales. Le pavillon d'extrémité abrite cuisine et abattoir. Le contraste est sensible avec la physionomie des porcheries avant 1914, qui ne se composaient que de trois simples séries de logettes accolées à la grange. De même, les modèles de râteliers-mangeoires, avec cheminée absorbant la poussière, proposés par Granet, se réfèrent à des propositions de « râteliers-abreuvoirs-mangeoires » également publiées dans les traités théoriques, notamment celui de Danguy. 
Figure 43

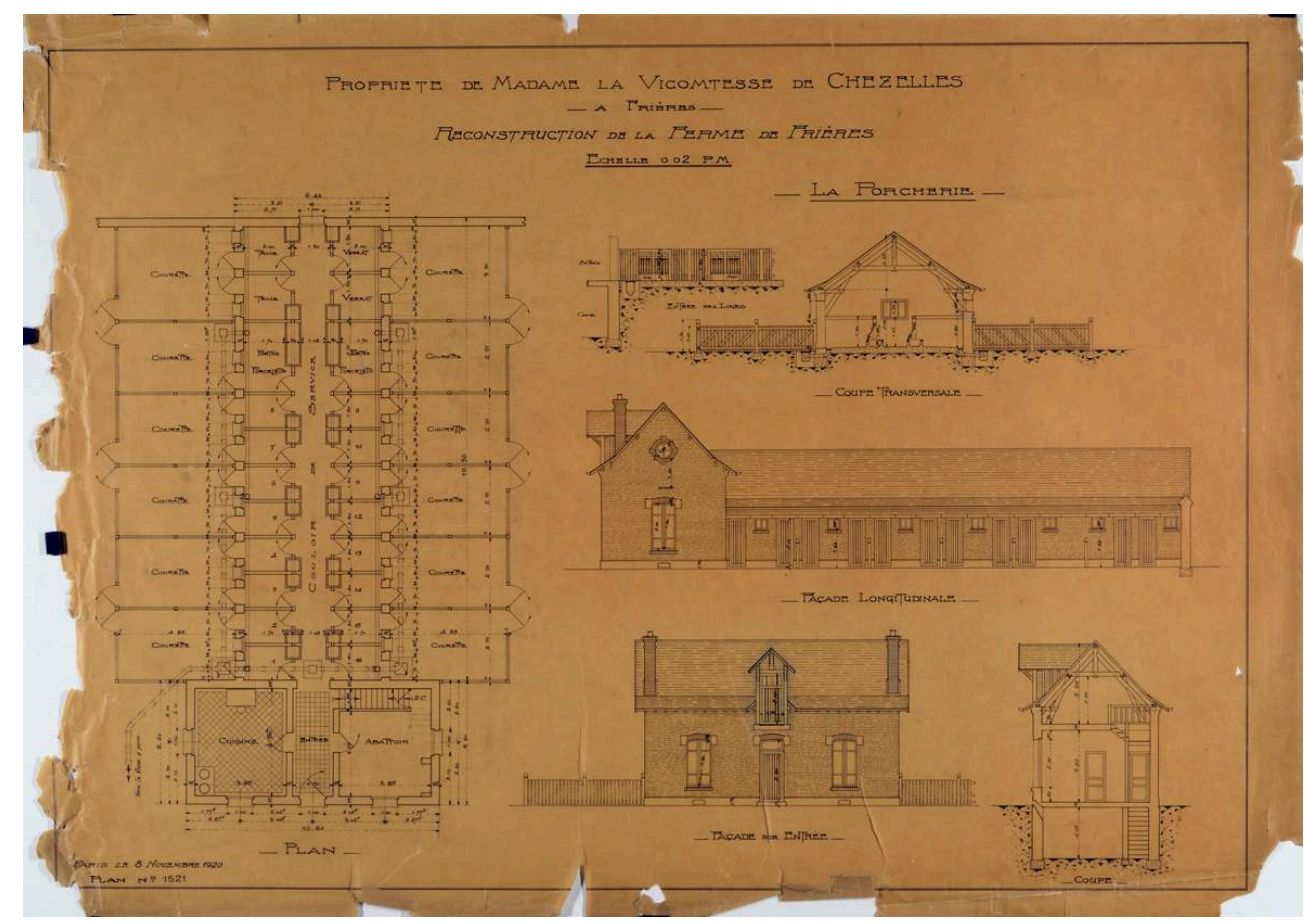

Projet de porcherie d'élevage de Frières, par André Granet, novembre 1920. SIAF, CAPA, fonds André Granet, 86 IFA 815.

(c) CAPA.

La triple vocation de cette grande ferme, associant polyculture céréalière, élevage et exploitation sucrière, impose l'existence de dispositifs spécifiques particulièrement consommateurs d'espaces bâtis, mais aussi de dégagements. C'est le cas des aménagements en rapport avec la culture betteravière, notamment la grande bouverie (fig. $\mathbf{n}^{\circ} \mathbf{4 4}$ ), qui occupe à elle seule l'un des angles de la cour, mais aussi les «silos à betteraves » établis tout à la fois à l'entrée de la ferme et à l'arrière de celle-ci, selon une disposition qui régularise celles en vigueur avant la guerre. À Villers-SaintChristophe et Aubigny-aux-Kaisnes, les interventions de Granet concernent également d'importantes exploitations sucrières. 
Figure 44

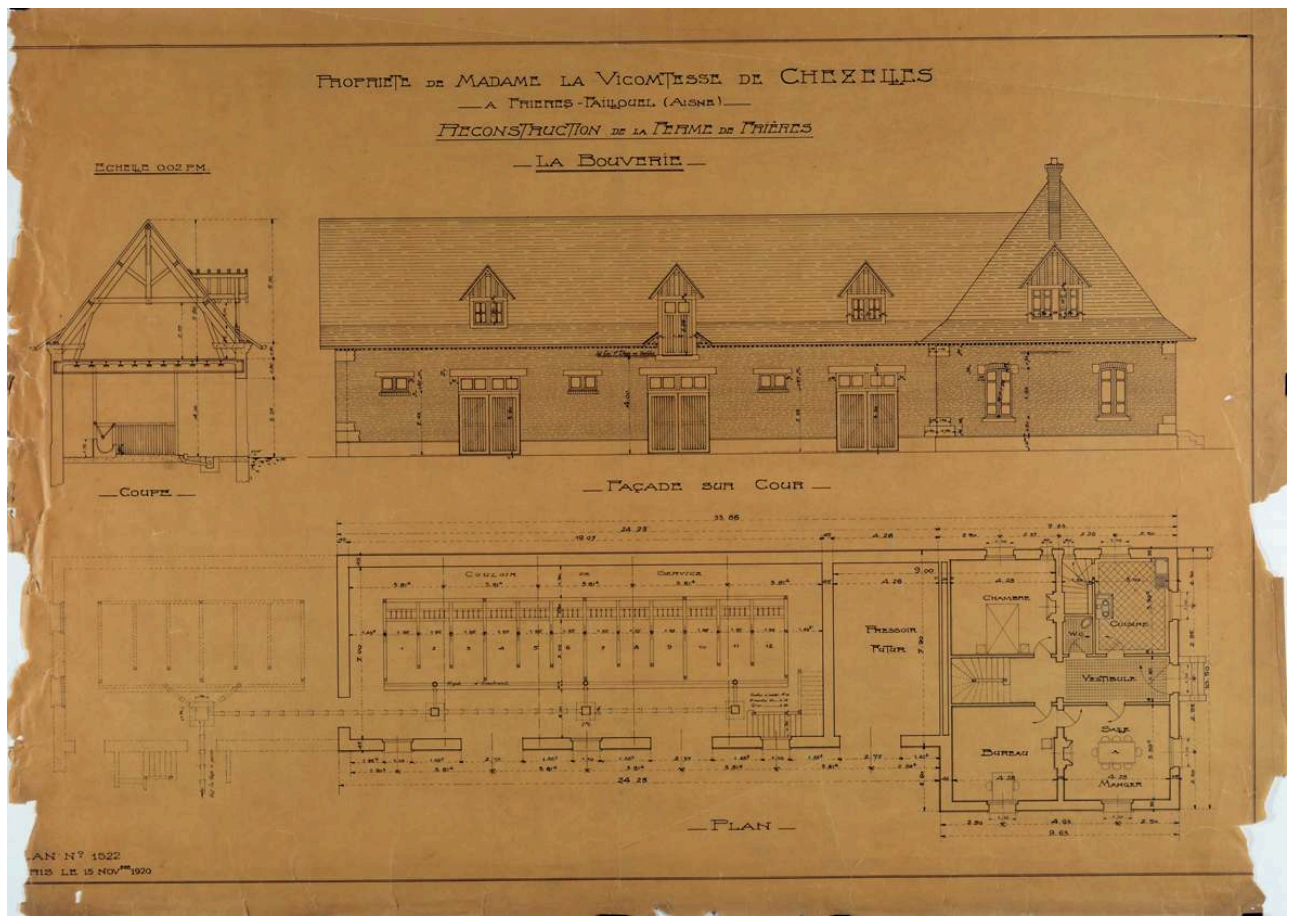

Projet de bouverie et de silos à betteraves, par André Granet, novembre 1920. SIAF, CAPA, fonds André Granet, 86 IFA 815

(c) CAPA.

\section{Figure 45}

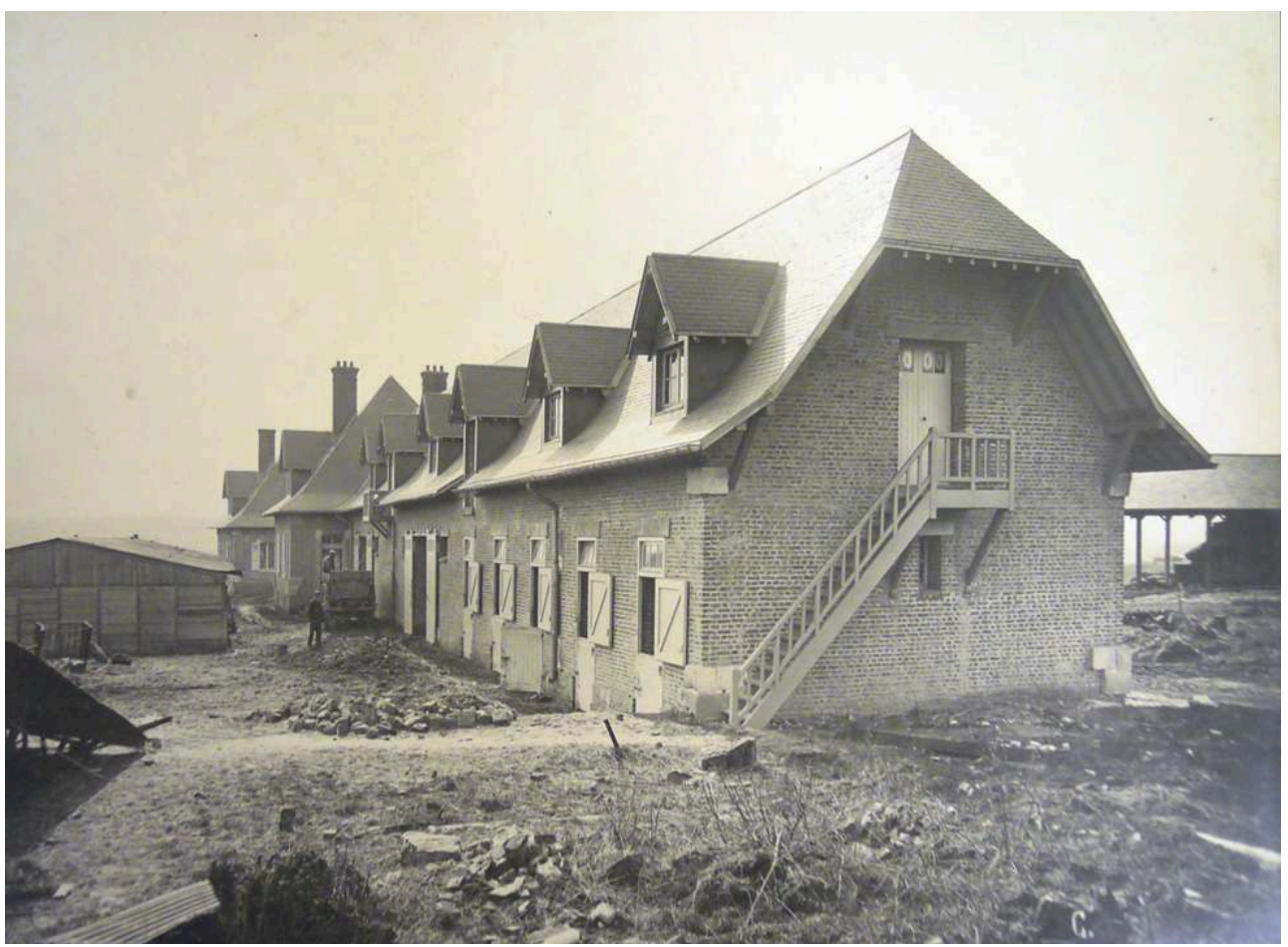

Le bâtiment abritant les écuries en cours d'achèvement, photographie non datée. Cliché Chevojon. SIAF, CAPA, fonds André Granet, 86 IFA 478/8.

(c) CAPA. 
Enfin, les recherches de solutions d'écoulement et de rejet des urines animales et du purin occupent une place substantielle en 1920. Granet propose ainsi un projet de caniveaux avec dalles en béton armé et dallage latéral en brique et « cassons de briques à sec ». La presque totalité des bâtiments est reconstruite en maçonneries de brique de four avec couverture en tuile et en ardoise (fig. $\mathbf{n}^{\circ} \mathbf{4 5}$ ). L'ancien portail monumental réemployé n'axe désormais plus vers l'habitation du fermier, maintenant cantonnée aux seuls pavillons d'entrée, mais vers les écuries et les étables (fig. $\mathbf{n}^{\circ} \mathbf{4 6}$ ).

\section{Figure 46}

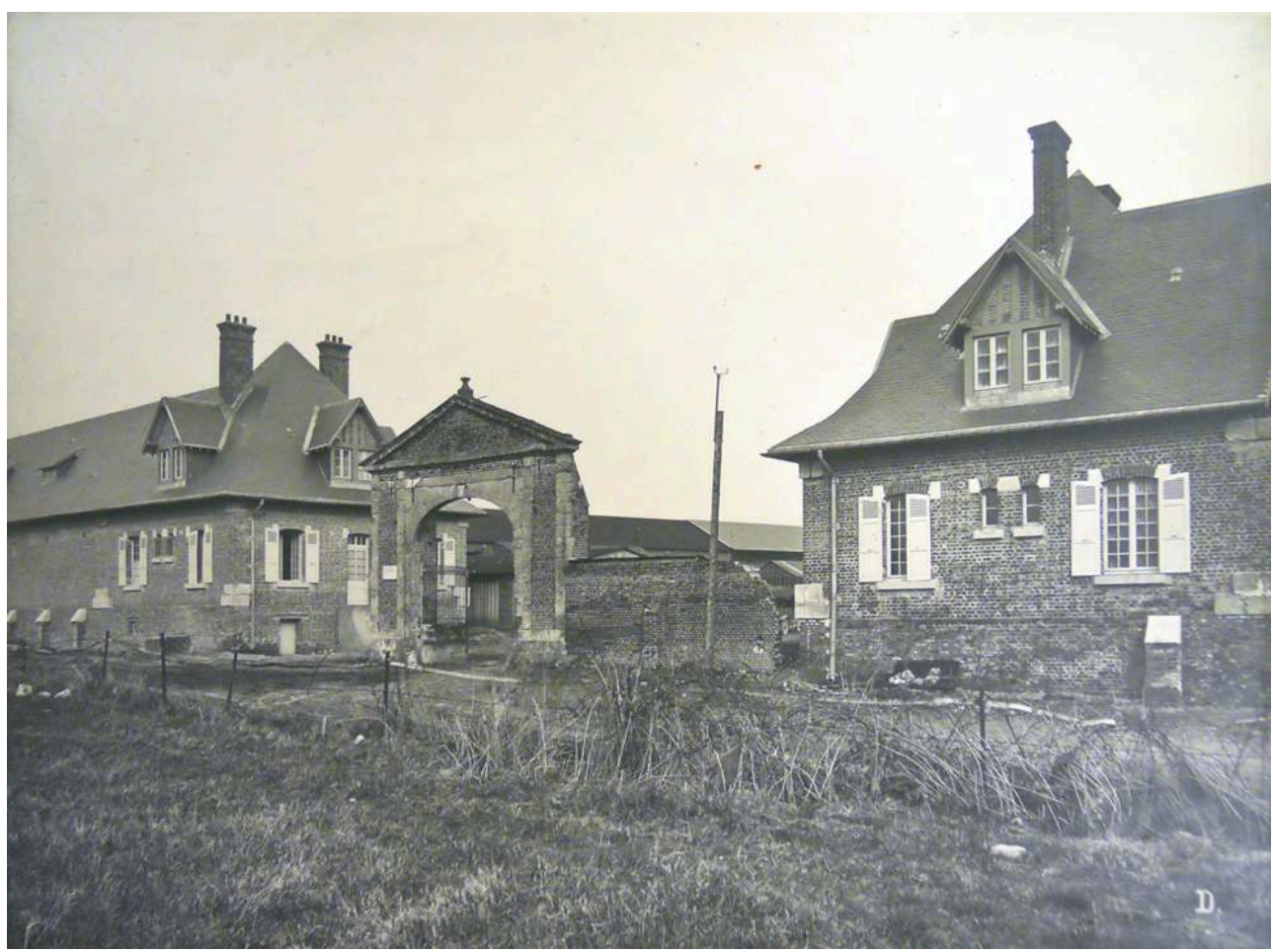

Les bâtiments de part et d'autre du portail d'entrée vus depuis la route, photographie non datée. Cliché Chevojon, SIAF, CAPA, fonds André Granet, 86 IFA 487/8.

(c) CAPA. 


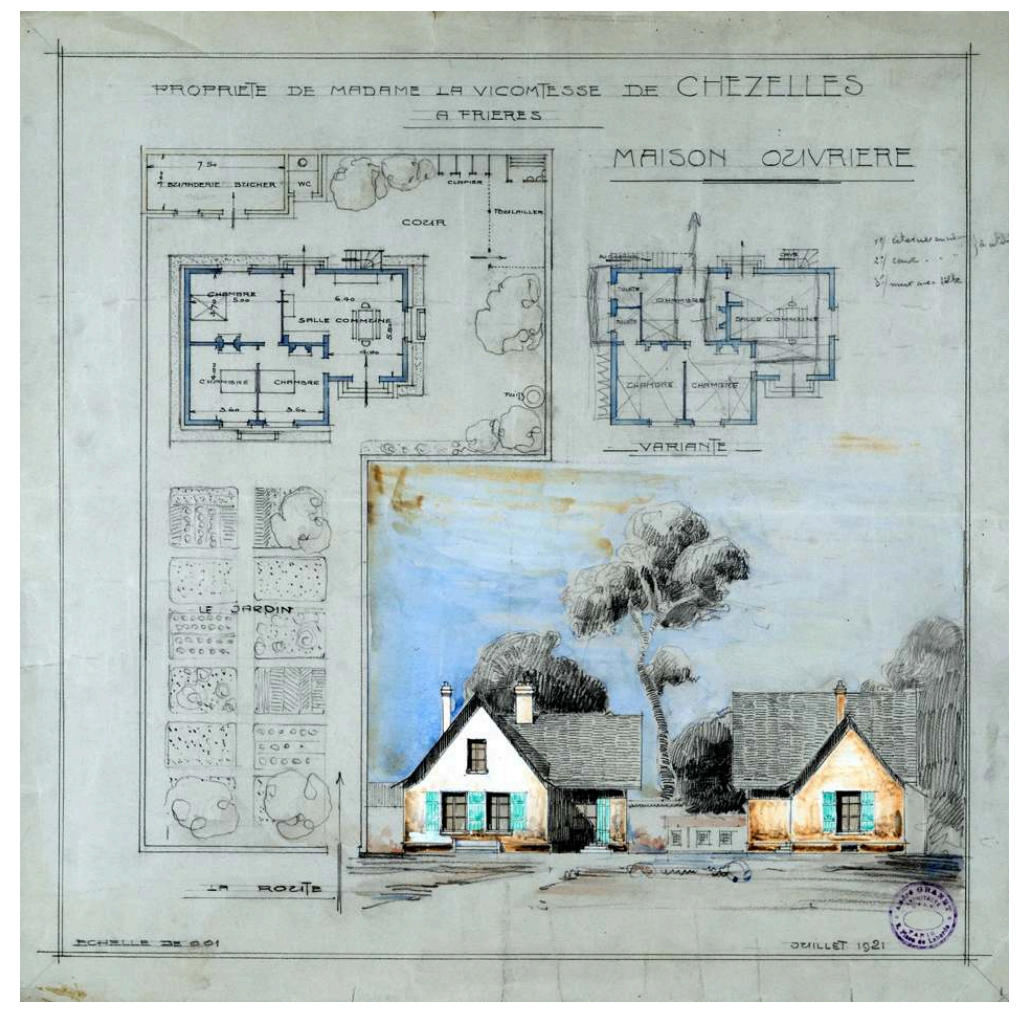

Projet de maisons ouvrières pour la ferme de Frières, par André Granet, juillet 1921. SIAF, CAPA, fonds André Granet, 86 IFA 812.

(C) CAPA.

En dernier lieu, Granet étudie une maison de régisseur mais aussi plusieurs projets de maisons ouvrières, individuelles ou à plusieurs logements (fig. $\mathbf{n}^{\circ} \mathbf{4 7}$ ) (fig. $\mathbf{n}^{\circ} \mathbf{4 8}$ ), qui peuvent être mis en rapport avec les projets types de Charles Letrosne publiés dans Murs et Toits pour les pays de chez nous (fig. $\mathbf{n}^{\circ}$ 49) et confrontés à d'autres réalisations relevant de la même typologie et inscrites dans le paysage de la reconstruction axonienne, comme la maison d'ouvrier agricole conçue par les architectes Raimbert et Papet pour la ferme de Margival à Montgarny (fig. $\mathbf{n}^{\circ}{ }^{50}$ ). Dans les trois cas, la conception architecturale et stylistique affirme la volonté d'inscription dans un discours régionaliste maîtrisé. 
Figure 48

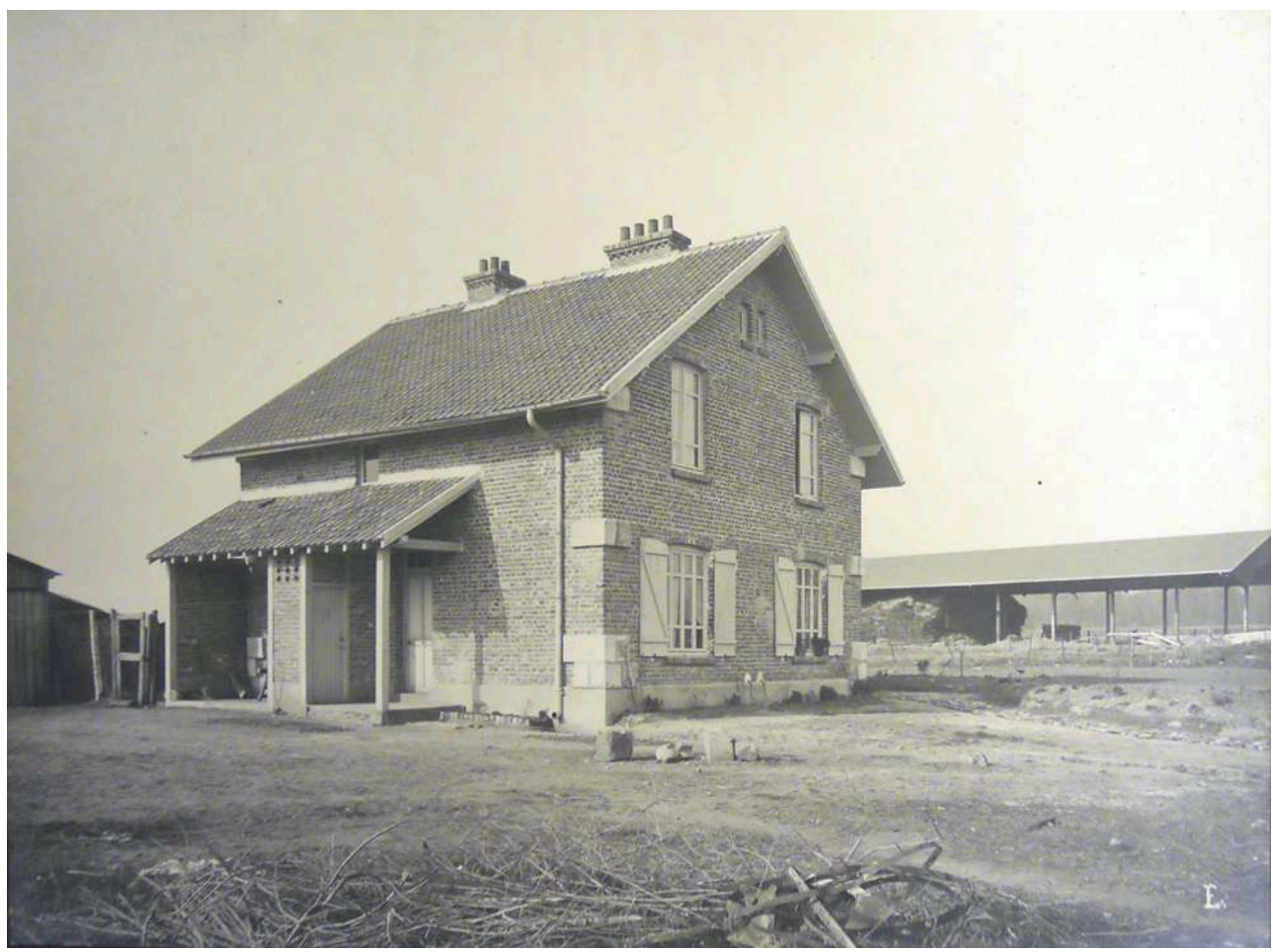

Maison ouvrière de la ferme de Frières, photographie non datée. Cliché Chevojon, SIAF, CAPA, fonds André Granet, 86 IFA 478/8.

(c) CAPA. 
Figure 49

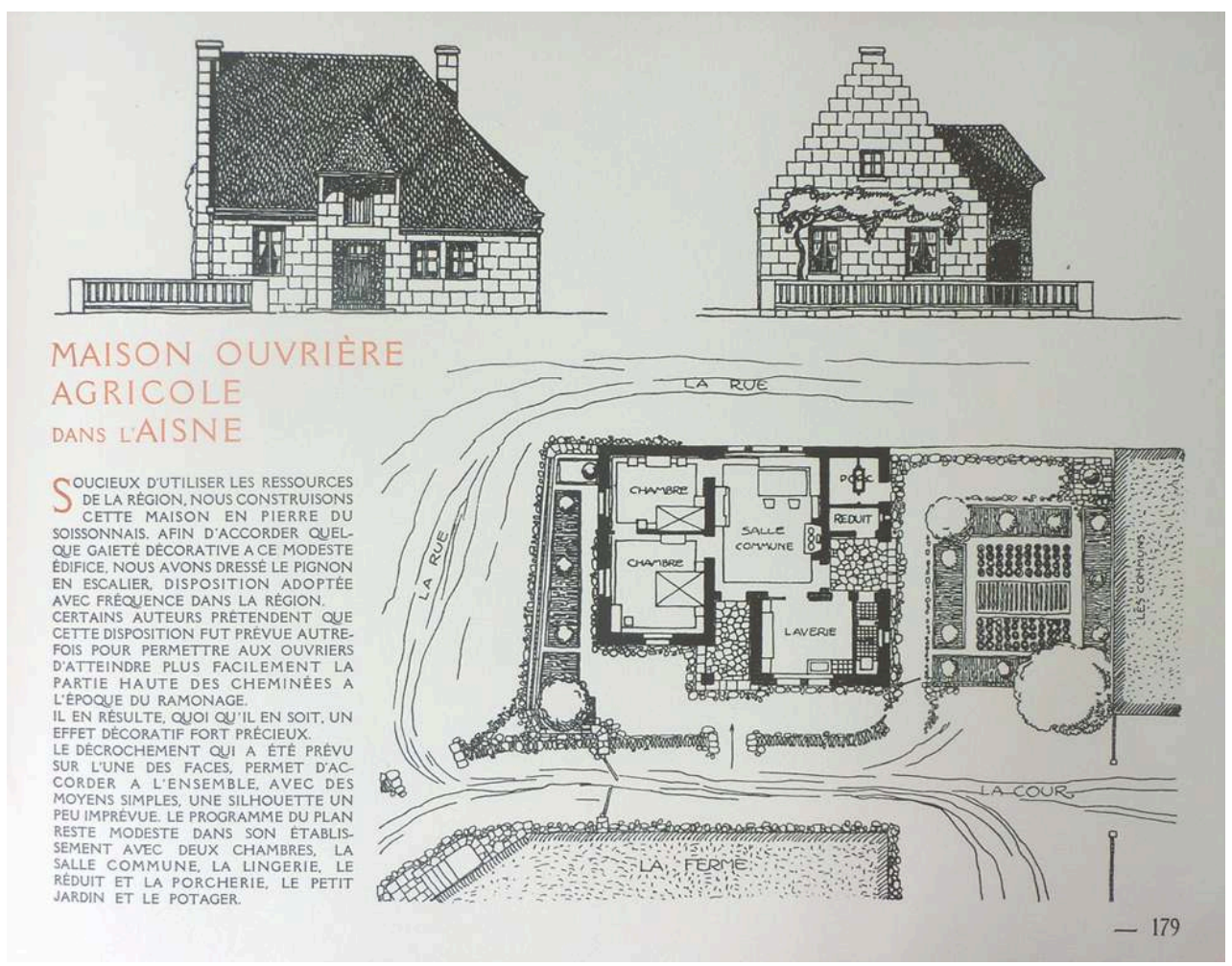

Maison ouvrière agricole dans l'Aisne publiée par Charles Letrosne dans Murs et toits pour pays de chez nous.

Phot. Delorme, Franck. (c) Franck Delorme.

Figure 50

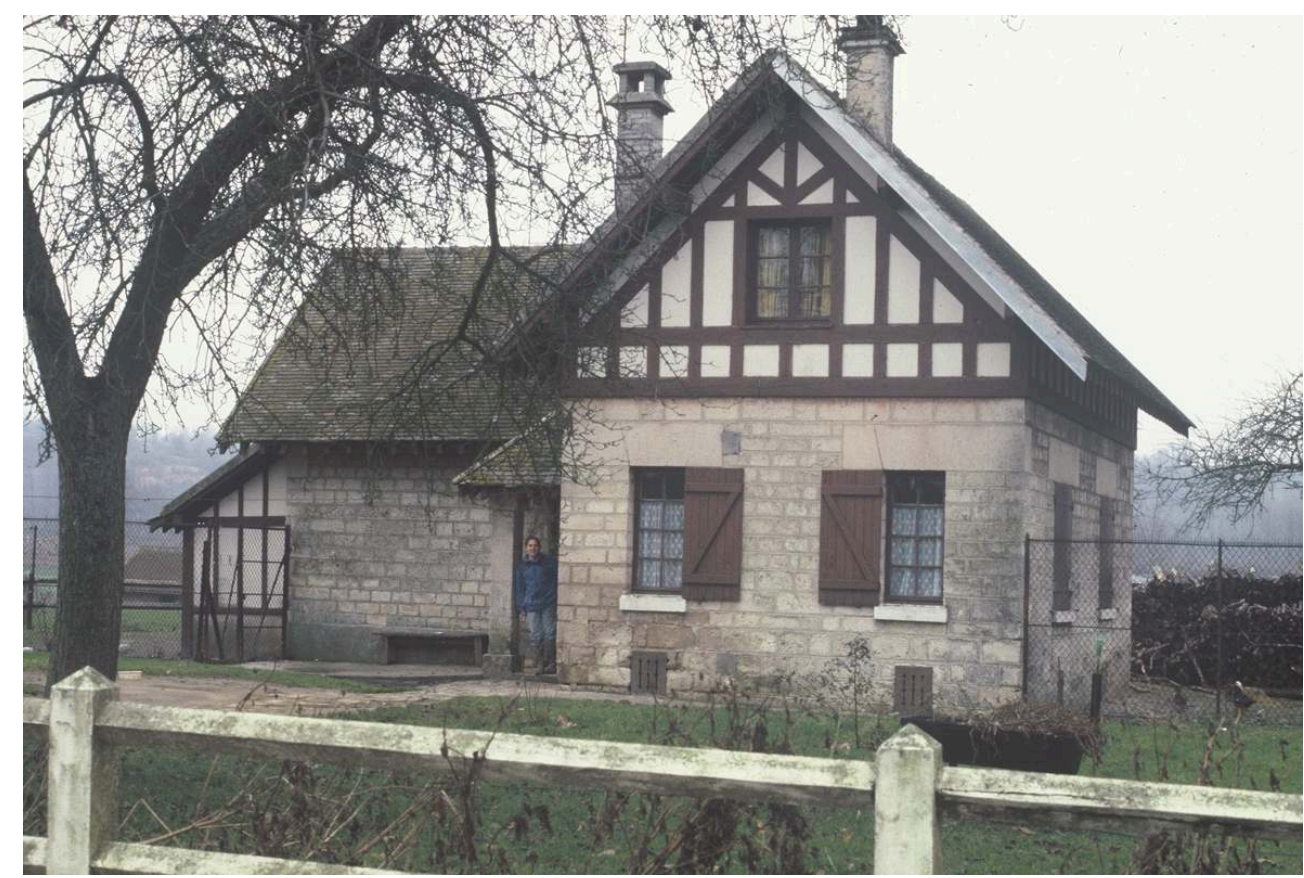

Maison ouvrière de la ferme de Montgarny à Margival dans l'Aisne.

Phot. Cappronnier Jean-Charles. (c) Jean-Charles Cappronnier. 


\section{Conclusion}

D'une manière générale, la reconstruction des fermes axoniennes à l'issue de la Première Guerre mondiale (fig. $\mathbf{n}^{\circ} \mathbf{5 1}$ ) révèle une grande stabilité dans les processus culturaux. En revanche, la reconstruction globale des exploitations, quelles que soient leurs tailles, manifeste, d'une part le souci de régulariser et de symétriser les dispositifs, d'autre part celui de concentrer les équipements et les services. L'abondance des matériaux traditionnels et leur coût modique - brique en Vermandois, moellons calcaires et pierre de taille en Soissonnais - permettent une économie de moyens pérennisant les mises en œuvre antérieures à 1914. En revanche, les prescriptions hygiénistes - ségrégation des espaces réservés aux hommes et aux animaux, aération et ventilation des bâtiments, dégagements et passages facilitant la circulation des charrois et des engins - demeurent parcimonieusement appliquées. Par ailleurs, l'ambiguité fondamentale de la législation sur les dommages de guerre, dont l'article 12 de la loi du 17 avril 1919 stipule que «l'indemnité consiste dans les sommes nécessaires à la reconstruction d'un édifice présentant le même caractère, ayant la même importance, la même destination et offrant les mêmes garanties de durée que l'immeuble détruit ", ne favorise guère l'innovation. La priorité accordée à la remise en état rapide des sols et des exploitations ne permet donc pas d'anticiper sur les méthodes nouvelles vantées par Compère-Morel. La "ferme de demain " n'est pas encore pour demain.

Figure 51

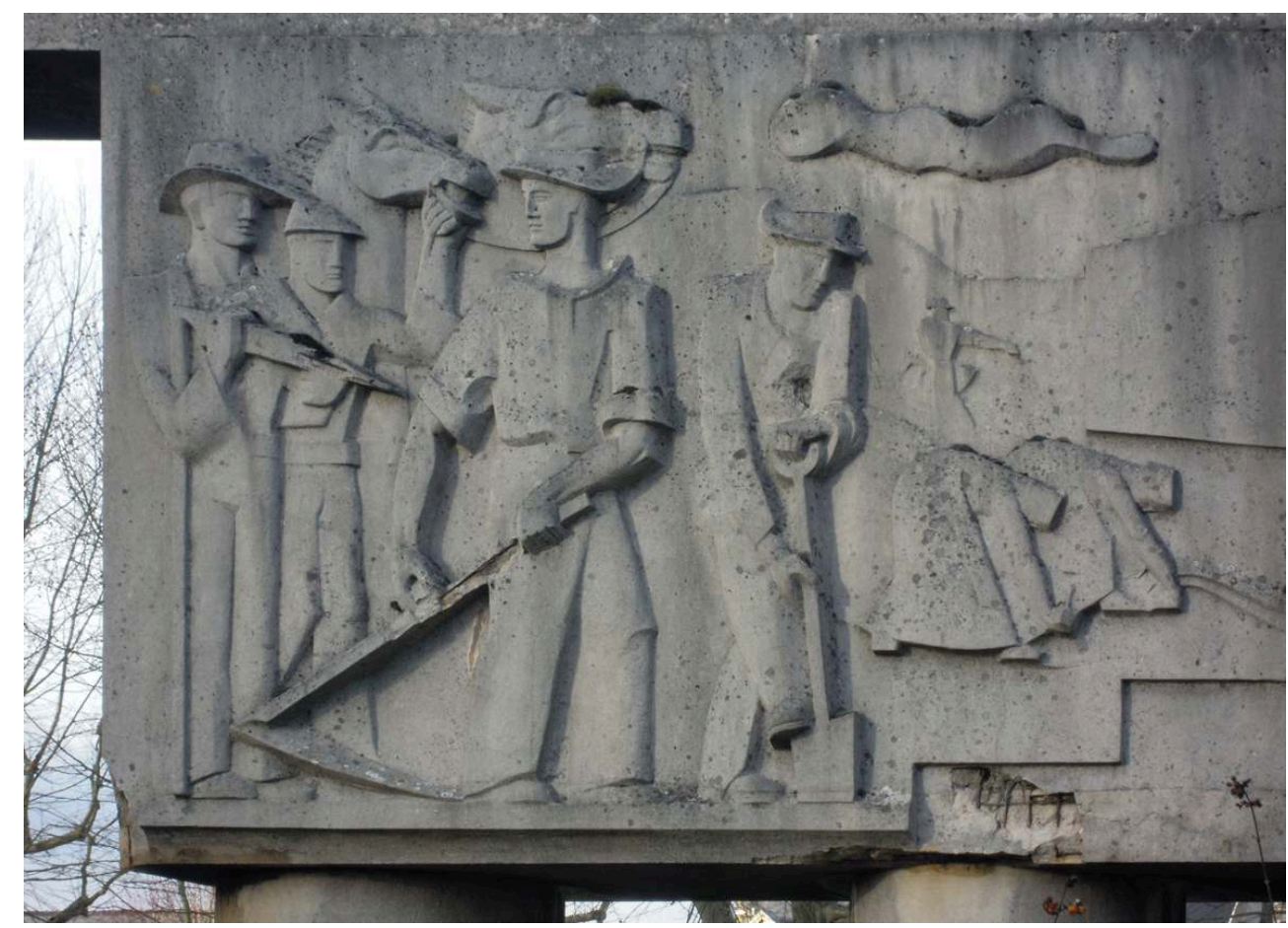

Détail du monument des Coopératives de reconstruction à Soissons dans l'Aisne.

Phot. Cappronnier Jean-Charles. (C) Jean-Charles Cappronnier. 


\section{NOTES}

1. - ROLLAND, Denis. Architectures rurales en Picardie. Le Soissonnais. Saint-Étienne : Éditions Créer, 1998.

2. - Sur une frontière de la France. La Thiérache. Aisne. Inventaire général des monuments et des richesses artistiques de la France / Association pour la généralisation de l'Inventaire régional en Picardie, 2001, p. 45.

3. - DORGELÈS, Roland. Le Réveil des morts. Paris : Albin Michel, 1923.

4. - Conservés à l'ECPAD. L'officier photographe Jean Tournassoud (1866-1951) ne prend la tête du Service photographique de l'armée qu'en 1918, mais exerce dès 1914 ses missions photographiques au sein des unités successives auxquelles il est rattaché.

5. - DUMONT-WILDEN, Louis. "Après la guerre, la reconstitution du paysage français ». Le Flambeau, 14 août 1915, p. 331.

6. - Les projets primés sont publiés dans Fermes et Habitations rurales: projets primés au concours ouvert entre les architectes français. Paris : Massin, [1918], 3 vol., (44, 20 et 36 pl.).

7. - GUADET, Julien. Éléments et théorie de l'architecture. T. 4, chap. 2, « Architecture rurale ». Paris : Librairie de la Construction moderne, s.d.

8. - TOULIER, Christine, HERMANOVICZ, Mariusz. Neuvy-le-Roi, ferme dite de Platé. Orléans : Inventaire région Centre, 1991.

9. - L'École nationale supérieure agronomique, située à Grignon, est fondée en 1826 par Ambroise Polycarpe de La Rochefoucauld, duc de Doudeauville.

10. - BOUCHARD-HUZARD, Louis. Traité des constructions rurales et de leur disposition. Paris : veuve Bouchard-Huzard, 1858-1860, 3 vol.

11. - CARLIER, Émile. Types de constructions rurales. Culture mixte, fermes, écuries, étables, bergeries, celliers, granges, citernes, réservoirs, etc. Paris : Ducher et Cie, 1881.

12. - GRANDVOINET, Jules Alexandre. Traité élémentaire des constructions rurales. Paris : Librairie agricole de la Maison rustique, 1883.

13. - ABADIE, Mars. La Ferme moderne, traité des constructions rurales. Paris : Librairie Larousse, coll. «Bibliothèque rurale ", 1903.

14. - Pour une bibliographie complète de la littérature sur le thème des constructions rurales et agricoles, se reporter à la bibliographie sélective du colloque L'Art de bâtir aux champs, organisé par l'INHA, disponible à la page <lien vers http://www.inha.fr/spip.php?article3276> .

15. - NARJOUX, Félix. Histoire d'une ferme. Paris : C. Delagrave, 1882.

16. - RIVOALEN, Émile. «L'architecture aux salons de 1899 ». Dans La Construction moderne, $\mathrm{n}^{\circ} 18$, 6 mai 1899 , p. 376-378.

17. - LETROSNE, Charles. Murs et Toits pour pays de chez nous. Paris, Dan Niestlé, T. 3, chap. 12 « Les fermes ", 1926.

\section{RÉSUMÉS}

D'un point de vue géographique, le département de l'Aisne est entièrement artificiel ; son territoire est ainsi fait de la réunion d'une pointe détachée de l'île-de-France (Valois et Meldois), d'une partie de la Champagne (Brie champenoise) et du sud de la Picardie (Soissonnais, Laonnois, 
Thiérache et Vermandois). Cette agrégation administrative se double naturellement d'une mosaïque de terrains géologiquement différents : une hétérogénéité qui fait du département de l'Aisne, en ce qui concerne l'architecture rurale, un cas d'étude digne d'intérêt car présentant des caractères variés et marqués. Au cours du premier conflit mondial, l'Aisne, essentiellement rurale mis à part Soissons et Saint-Quentin, voit ses villages et ses fermes dévastés par les combats. C'est donc un terrain «idéal " pour la mise en application des réflexions portant sur l'amélioration de l'appareil productif agricole et de son architecture. L'organisation en 1916 de l'exposition de la "Cité reconstituée », la création en 1917 de l'office de reconstitution agricole (ORA) des régions envahies, et, enfin, le lancement la même année du concours de modèles types, faisant suite à l'exposition d'architecture régionale, vont être trois facteurs préalables et déterminants pour engager une véritable pensée sur l'architecture agricole. Cette réflexion et ses applications sur le terrain ne font en réalité que prolonger le mouvement de renouvellement et d'amélioration agronomiques engagé bien avant la guerre, dès la fin du XIX ${ }^{\mathrm{e}}$ siècle. Les tentatives y furent nombreuses pour engager les grandes exploitations agricoles sur la voie d'une plus grande rationalité. Les exemples de fermes modèles des années 1880 et 1890 parsèment le territoire français. Les enjeux de ces réflexions sont multiples et ne portent pas seulement sur la rationalisation. Si le remembrement foncier, certes limité, et le transfert des dommages de guerre permettent la concentration de l'appareil productif agricole, la mise en place de normes d'hygiène touche aussi les petites exploitations familiales. Les architectes contribuent fortement à ces évolutions. Les fermes de l'Aisne, dans leur diversité de taille et de vocation, sont un vaste champ d'expérimentation tant du point de vue de la rationalisation (dans les grandes exploitations de plateau), du point de vue de l'hygiène (dans les petites exploitations regroupées en villages) que dans la définition d'une architecture de prestige (grands domaines aristocratiques).

From a geographical point of view the Aisne department is completely artificial; the territory consists of the merging of a detached tip of Île-de-France (Valois and Meldois), a part of Champagne (Brie Champenoise) and the south of Picardie (Soissonnais, Laonnois, Thiérache and Vermandois). This administrative aggregation naturally goes hand in hand with a mosaic of terrains which differ geologically. It is this very heterogeneity which makes the Aisne department, as regards rural architecture, a case study worthy of interest as it displays varied and pronounced characteristics. During the First World War, the Aisne, essentially rural with the exception of Soissons and Saint-Quentin, saw its villages and farms devastated in the fighting. It is therefore an "ideal" field for implementing deliberations concerning the improvement of agricultural productive capacity and its architecture.

\section{INDEX}

Mots-clés : Aisne, appareil productif agricole, architecte reconstructeur, architecture agricole, Marcel Boille, Brie champenoise, Bucy-le-Long, Cité reconstituée, coopératives de reconstruction, dommages de guerre, Ecole nationale d'agriculture, Gaston Ernest, exploitation agricole, ferme Agricola, ferme de grande culture, ferme expérimentale, ferme modèle, ferme moderne, FrièresFaillouël, André Granet, Grignon, Julien Guadet, hygiène, Laonnois, Camille Lefèvre, Charles Letrosne, Leuilly-sous-Coucy, ministère des Régions libérées, modèles types, Félix Narjoux, office de reconstitution agricole, Parcy-et-Tigny, plan d'aménagement d'embellissement et d'extension, Première Guerre mondiale, rationalisation, reconstruction rurale, remembrement foncier, Paul Ernest Sanson, Soissonnais, Thiérache, Valois, Vermandois, Vierzy, Eugène Emmanuel Viollet-leDuc 


\section{AUTEURS}

\section{JEAN-CHARLES CAPPRONNIER}

Docteur en histoire de l'architecture, Conservateur du patrimoine

jeancharles.cappronnier@dbmail.com

\section{FRANCK DELORME}

Historien de l'architecture, attaché de conservation à la Cité de l'architecture et du patrimoine fdelorme@citechaillot.fr 